Aus der Klinik für Gastroenterologie und gastrointestinale Onkologie

(Prof. Dr. med. V. Ellenrieder)

der Medizinischen Fakultät der Universität Göttingen

\title{
Einfluss der Nahrungsaufnahme auf die Lebersteifigkeit, gemessen mit 2D- Scherwellen-Elastographie (Gerät: LOGIQ E9) bei freiwilligen Probanden
}

\author{
INAUGURAL-DISSERTATION \\ zur Erlangung des Doktorgrades \\ der Medizinischen Fakultät der \\ Georg-August-Universität zu Göttingen
}

vorgelegt von

Melissa Porsche

aus Bochum 
Dekan:

\section{Betreuungsausschuss}

Betreuer:

Ko-Betreuer:

\section{Prüfungskommission}

Referent/in

Ko-Referent/in:

Drittreferent/in:
Prof. Dr. rer. nat. H. K. Kroemer

PD Dr. Dr. med. A. Neeße

PD Dr. med. H. Rosewich

Datum der mündlichen Prüfung: 
Hiermit erkläre ich, die Dissertation mit dem Titel "Einfluss der Nahrungsaufnahme auf die Lebersteifigkeit, gemessen mit 2D-Scherwellen-Elastographie (Gerät: LOGIQ E9) bei freiwilligen Probanden" eigenständig angefertigt und keine anderen als die von mir angegebenen Quellen und Hilfsmittel verwendet zu haben.

Göttingen, den

(Melissa Porsche)

Teile dieser Dissertation wurden im Februar 2019 in Ultrasound in Medicine and Biology als folgendes Manuskript publiziert:

Petzold G, Porsche M, Ellenrieder V, Kunsch S, Neesse A (2019): Impact of Food Intake on Liver Stiffness Determined by 2-D Shear Wave Elastography: Prospective Interventional Study in 100 Healthy Patients. Ultrasound Med Biol $\underline{45}$, 402-410 


\section{Inhaltsverzeichnis}

Abkürzungsverzeichnis III

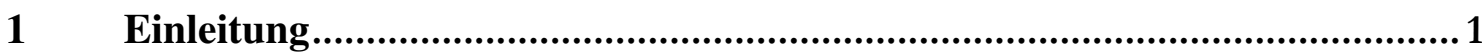

1.1 Chronische Lebererkrankungen und Diagnostik der Leberfibrose ........................................1

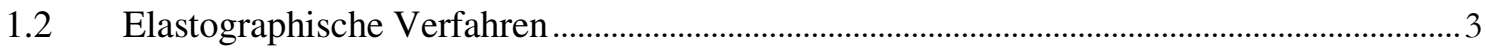

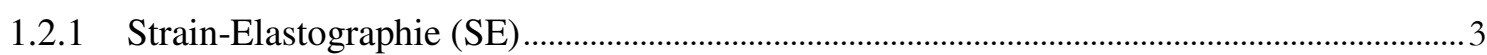

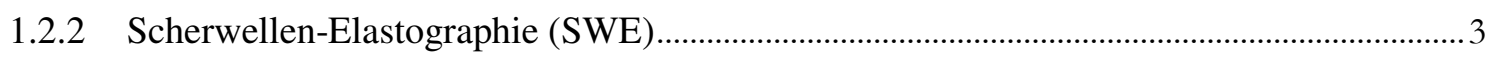

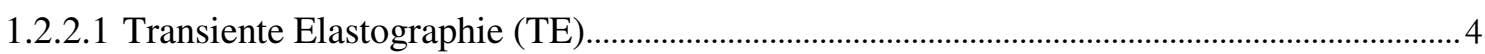

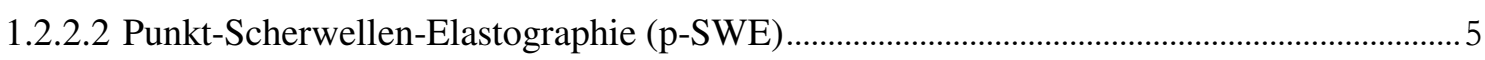

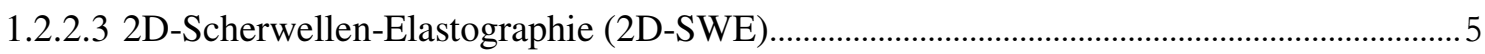

1.3 Einflussfaktoren der Scherwellen-Elastographie ............................................................

1.3.1 Nahrungsaufnahme und Scherwellen-Elastographie............................................................

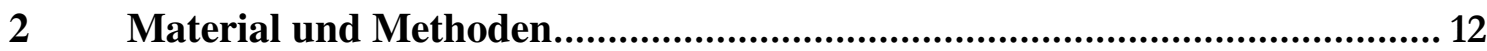

2.1 Studiendesign und Patientenkollektiv................................................................................12

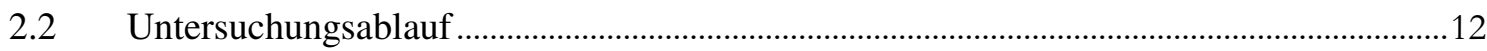

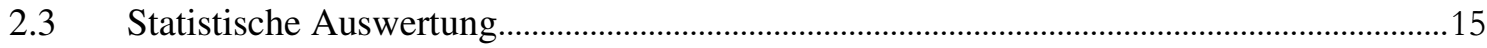

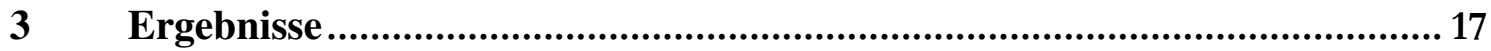

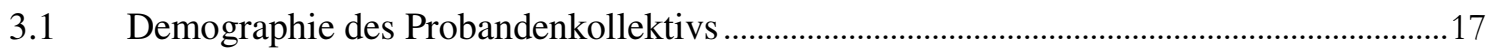

3.2 Lebersteifigkeit (2D-SWE) bei lebergesunden Probanden.....................................................19

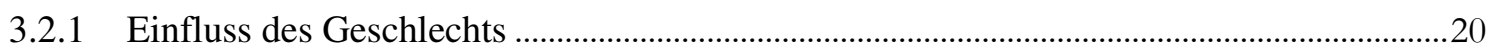

3.2.2 Einfluss des Alters, des BMIs und des Alkoholkonsums......................................................21

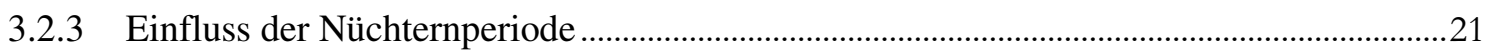

3.2.4 IQR-Wert und der Einfluss des Geschlechts, der Nüchternperiode und des LSAusgangswerts …………………………………………………………………………....22

3.3 Abhängigkeit der Lebersteifigkeit (2D-SWE) von der Nahrungsaufnahme.........................23

3.3.1 Einfluss des Geschlechts auf den postprandialen LSA .....................................................24

3.3.2 Einfluss des Alters und des BMIs auf den postprandialen LSA............................................24

3.3.3 Einfluss der Medikamenteneinnahme und des Alkoholkonsums auf den

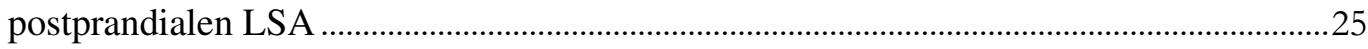

3.3.4 Einfluss der Nüchternperiode auf den postprandialen LSA ……………………..................26

3.3.5 Einfluss des LS-Ausgangswerts auf den postprandialen LSA ………..................................27

3.3.6 Fibrosestadien des Probandenkollektivs gemäß definierten Cut-off-Werten ......................28

3.4 Abhängigkeit des IQR-Werts von der Nahrungsaufnahme ................................................29

3.5 Abhängigkeit des Pfortaderblutflusses von der Nahrungsaufnahme .....................................29

3.6 Abhängigkeit des Gallenblasenvolumens von der Nahrungsaufnahme.................................31

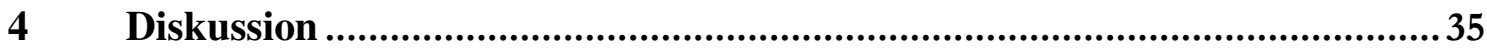

4.1 Lebersteifigkeit (2D-SWE) bei lebergesunden Probanden.......................................................35

4.2 Abhängigkeit der Lebersteifigkeit (2D-SWE) von der Nahrungsaufnahme........................36 
4.2.1 Einfluss des LS-Ausgangswerts auf den postprandialen LSA .............................................39

4.2.2 Einfluss des Geschlechts, des Alters, des BMIs, des Alkoholkonsums, der Medikamenteneinnahme und der Nüchternperiode auf den postprandialen LSA ..............39

4.3 Abhängigkeit des Pfortaderblutflusses von der Nahrungsaufnahme ......................................40

4.4 Abhängigkeit des IQR-Werts von der Nahrungsaufnahme ....................................................42

4.5 Abhängigkeit des Gallenblasenvolumens von der Nahrungsaufnahme ................................42

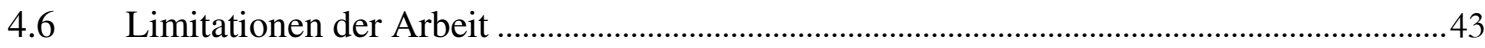

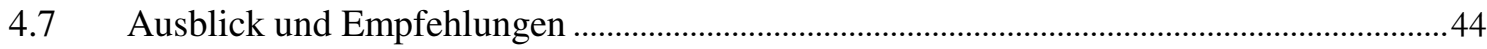

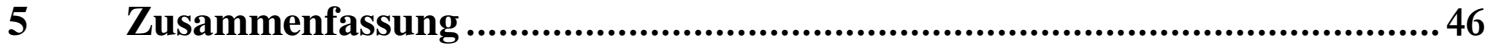

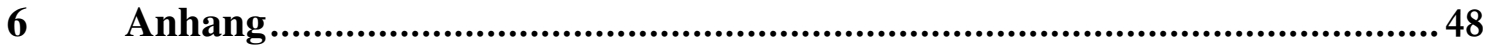

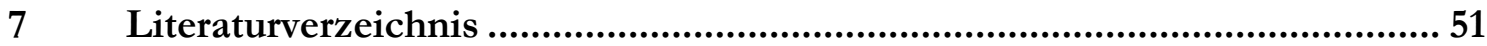




\section{Abkürzungsverzeichnis}

2D-SWE 2D-Scherwellen-Elastographie

$\begin{array}{ll}\text { ARFI } & \text { acoustic radiation force imaging } \\ \text { b } & \text { Regressionskoeffizient } \\ \text { BMI } & \text { body mass index } \\ \text { CDT } & \text { carbohydrate deficient transferrin } \\ \text { E } & \text { Elastizität } \\ \text { GBV } & \text { Gallenblasenvolumen } \\ \text { HCV } & \text { Hepatitis-C-Virus } \\ \text { HSC } & \text { hepatic stellate cell }=\text { hepatische Sternzelle } \\ \text { ICR } & \text { Intercostalraum } \\ \text { IQR } & \text { interquartile range }=\text { Interquartilsabstand } \\ \text { LS } & \text { Lebersteifigkeit }\end{array}$

LSA Lebersteifigkeitsanstieg

LSM Lebersteifigkeitsmessung

MCV Mittleres Erythrozyten-Volumen

NAFLD non-alcoholic fatty liver disease $=$ nicht-alkoholische Fettlebererkrankung

p-SWE Punkt-Scherwellen-Elastographie

PBF portaler Blutfluss

r Korrelationskoeffizient

ROI region of interest

SD $\quad$ standard deviation $=$ Standardabweichung

SE Strain-Elastographie

SWE Scherwellen-Elastographie

TE transiente Elastographie

V Ausbreitungsgeschwindigkeit

$\rho \quad$ Dichte 


\section{$1 \quad$ Einleitung}

\subsection{Chronische Lebererkrankungen und Diagnostik der Leberfibrose}

Die Leber ist ein zentrales und lebenswichtiges Organ des menschlichen Körpers. Speicherung und Ausscheidung von Stoffwechselprodukten, Biotransformation und Synthese von Gerinnungsfaktoren sind nur einige Stoffwechselvorgänge, in denen die Leber eine entscheidende Rolle spielt (Gekle 2009). Eine Voraussetzung, um diese Aufgaben zu erfüllen, ist ein gesundes Organ. Aus einigen Erkrankungen der Leber, wie zum Beispiel viralen Hepatitiden, kann demnach eine Insuffizienz ihrer Funktion resultieren. Insbesondere bei Chronifizierungen von Lebererkrankungen kann es zu einer Vermehrung der extrazellulären Matrix und damit zu einer Fibroseentstehung kommen (Sebastiani et al. 2014). Eine zentrale Rolle spielen hierbei unter anderem die hepatischen Sternzellen (HSC), welche sich in einer gesunden Leber in der Ruhephase befinden, Retinoide speichern und saure Gliafaserproteine (GFAP) synthetisieren (Guyot et al. 2006; Kendall et al. 2009). Bei chronischen Lebererkrankungen kommt es durch komplexe autokrine und parakrine Mechanismen zu einer Aktivierung der HSC. Diese verlieren daraufhin langsam ihre Speicherfunktion und entwickeln sich zu Myofibroblasten, was zu einer vermehrten Produktion von extrazellulärer Matrix führt (Elpek 2014).

$\mathrm{Zu}$ den häufigsten Ursachen der Entwicklung einer Leberfibrose zählen vor allem die alkoholische und die nicht-alkoholische Fettlebererkrankung sowie eine Infektion mit dem Hepatitis B- oder C-Virus (Guyot et al. 2006; Kendall et al. 2009). Zudem kann eine Leberfibrose auch aufgrund von Autoimmunprozessen oder im Rahmen von Stoffwechselerkrankungen wie Morbus Wilson oder der Hämochromatose entstehen (Crownover und Covey 2013; Poujois und Woimant 2018). Auch Erkrankungen wie die primäre biliäre Cholangitis (PBC) können eine Leberfibrose oder Zirrhose zur Folge haben (Poupon 2015).

Durch Voranschreiten der Erkrankungen bzw. der Fibrose kann sich im Verlauf eine Zirrhose entwickeln (Pinzani und Macias-Barragan 2010). Jährlich versterben weltweit über 1,2 Millionen Menschen an einer Leberzirrhose und ihren Komplikationen (Wang et al. 2016). Die frühzeitige Diagnose des Fibrosegrades bei Patienten mit chronischen Lebererkrankungen ist ausschlaggebend für die Therapieplanung, die Erkrankungsprogression und die Prognose (Dietrich et al. 2017). Der derzeitige Goldstandard in der Fibrose- und Zirrhosediagnostik ist die Leberbiopsie (Rockey et al. 2009). Dies ist allerdings ein invasives Verfahren, welches potenziell einige Komplikationen wie Blutungen oder Infektionen mit sich bringen kann (Strobel et al. 2015). Außerdem wird die gesamte Leber mittels einer Stanzbiopsie nur durch einen kleinen Gewebszylinder repräsentiert. Unter anderem aufgrund dessen 
kann es zu Fehlklassifikationen der Fibrose/Zirrhose kommen. Nur 65-75 \% der Leberbiopsien mit einer Zylindergröße von 15 - 25 mm klassifizieren den Fibrosegrad korrekt nach dem METAVIR-Score (Bedossa et al. 2003). Ein weiterer Faktor, der die Biopsie in ihrer diagnostischen Genauigkeit limitiert, ist die hohe Intra- und Interbeobachtervariabilität (Baunsgaard et al. 1979; Regev et al. 2002).

Dies führte dazu, dass nach alternativen Methoden in der Fibrosediagnostik gesucht und in den vergangenen Jahren viel Forschung auf diesem Gebiet betrieben wurde. Im Vordergrund standen hierbei vor allem Verfahren zur Weiterentwicklung radiologischer und sonographischer Verfahren. Die Sonographie ermöglicht es, einen großen Bereich der Leber nicht-invasiv zu untersuchen und durch Echogenitätsunterschiede Pathologien zu detektieren. Eine Hypertrophie des Lobus caudatus, Veränderungen der Leberoberfläche und des hepatisch venösen Blutflusses können auf eine Zirrhose hinweisen, wobei die Beurteilung der Leberoberfläche die höchste diagnostische Genauigkeit zeigt (Colli et al. 2003). In bis zu $85 \%$ der Patienten mit chronischen Lebererkrankungen ist die Detektion einer Zirrhose mittels BBild-Sonographie möglich, jedoch ist die Präzision in der Diagnostik durch anatomische Gegebenheiten und vor allem durch die Untersucherabhängigkeit begrenzt (Aubé et al. 1999). Ein weiterentwickelter Ansatz mit sonographischem Hintergrund ist die Elastographie. Dabei handelt es sich um eine Messung der Gewebselastizität bzw. Gewebssteifigkeit. Hierbei wurde sich zunutze gemacht, dass während eines chronischen Prozesses, wie der Fibrose, das Lebergewebe an Steifigkeit zunimmt und an Elastizität verliert (Ferraioli et al. 2015). Schon im 17. Jahrhundert v. Chr. wurde die Palpation zur Beurteilung der Gesundheit von menschlichen Organen genutzt (Wilkins 1964). Es galt die Annahme, dass sich gesundes und krankes Gewebe hinsichtlich ihrer Steifigkeit und Elastizität voneinander unterscheiden (Garra 2015). Und auch heute spielt die Palpation bei der klinischen Untersuchung eine wichtige Rolle. Allerdings ist es nur möglich, von der Oberfläche aus erreichbare Organe zu beurteilen. Die Elastographie stellt eine nicht-invasive Methode dar, die es erlaubt, auch tieferliegende Organe und Strukturen bezüglich ihrer visko-elastischen Eigenschaften zu untersuchen und kann als eine Art „elektronische Palpation“ angesehen werden (Bamber et al. 2013). Die Elastographie ist ein kostengünstiges und schnelles Verfahren ohne Strahlenbelastung und bietet im Vergleich zu vielen anderen Methoden den Vorteil, dass sie aufgrund ihrer nicht vorhandenen Invasivität vielfach wiederholt werden kann. Daher spielt die Leberelastographie nicht nur in der Diagnostik, sondern potenziell auch in Verlaufskontrollen und zur Beurteilung von Therapieerfolgen bei Patienten mit chronischen Lebererkrankungen eine Rolle (Friedrich-Rust und Vermehren 2013). Elastographie wird neben der Leberfibrosediagnostik auch für die Beurteilung von Pathologien anderer Organe wie Schilddrüse, Prostata, Lymphknoten und Milz verwendet. Ebenfalls findet die Elastographie Anwendung in der Diagnostik von muskuloskelettalen Erkrankungen (Cosgrove et al. 2013). Bei den folgenden Erläuterungen liegt der Schwerpunkt auf Erkrankungen der Leber. 


\subsection{Elastographische Verfahren}

Es existieren mehrere Elastographieverfahren, die entweder anhand der Methode, mit welcher der Impuls appliziert wird, oder anhand der verwendeten Messtechnik der Gewebsdeformation klassifiziert werden können (Treece et al. 2011). Alle Methoden haben gemeinsam, dass sie mittels Ultraschall Gewebsverschiebungen oder Scherwellengeschwindigkeiten detektieren und daraus Elastizitätsmessungen oder Elastizitätsbilder (Elastogramme) generieren (Bamber et al. 2013).

\subsubsection{Strain-Elastographie (SE)}

Eine quasi-statische Methode, bei der die Gewebsverschiebung durch externen Druck unter Verwendung einer Sonde oder endogenen Drucks (zum Beispiel durch kardiovaskuläre Bewegungen) erzeugt wird, ist die Strain-Elastographie (SE) (Cosgrove et al. 2013). Durch manuelle Kompression des Gewebes mittels einer Sonde kommt es zu axialen Gewebsverschiebungen der zu untersuchenden Region. Die Bewegung des Untersuchungsbereichs wird mit einem festgelegten Ultraschall-Tracking-Algorithmus verfolgt. Die entstehenden Echosignale werden mit den Signalen des jeweilig vorherigen Bildes an mehreren Bildpunkten miteinander verglichen. Daraus wird im Anschluss die Verschiebung elektronisch errechnet. Eine bildliche Darstellung erfolgt, indem die Ergebnisse der Berechnung als Elastogramm über das konventionelle Ultraschall-B-Bild gelegt und in Grautönen oder farblich codiert angezeigt werden (Bamber et al. 2013; Cosgrove et al. 2013; Jiang und Hall 2015). Die Darstellung als Elastogramm ist eine semi-quantitative Abbildung und kann nicht direkt in die Lebersteifigkeit (LS) übersetzt werden (Cosgrove et al. 2013). Absolute Elastizitätswerte bleiben unbekannt (Franckenberg et al. 2016). Ein Vorteil dieser Messmethode ist, dass sie in Echtzeit erfolgt und eine hohe räumliche Auflösung bietet. Allerdings werden die Messergebnisse stark vom Untersucher beeinflusst und sind von Faktoren wie Atembewegungen und kardiopulmonalen Pulsationen abhängig (Cosgrove et al. 2013). Aktuell findet die Strain-Elastographie vor allem in der Diagnostik von Schilddrüsenerkrankungen Anwendung (Sun et al. 2014). In der Leberdiagnostik spielt die SE eine untergeordnete Rolle (Cosgrove et al. 2013).

\subsubsection{Scherwellen-Elastographie (SWE)}

Neben der quasi-statischen Strain-Elastographie haben sich einige weitere Methoden etabliert, die sich dynamische Verfahren nennen. Dazu zählen die transiente Elastographie (TE), die Punkt-Scherwellen-Elastographie (p-SWE, ARFI quantification) und die 2D-Scherwellen-Elastographie (2D-SWE). All diesen Verfahren ist gemein, dass Scherwellen generiert werden. Mithilfe der Geschwindigkeitsanalyse dieser Schwerwellen können quantitative 
Aussagen über die Gewebselastizität getroffen werden, denn die Ausbreitungsgeschwindigkeit einer Scherwelle und die Gewebselastizität sind über Youngs Elastizitätsmodul ( $\mathrm{E}=3$ $\rho V^{2}$ ) miteinander verknüpft (Bamber et al. 2013; Cosgrove et al. 2013; Dietrich et al. 2017).

Scherwellen sind genauso wie konventionelle Ultraschallwellen mechanische Wellen, die sich im Gewebe ausbreiten können. Es gibt allerdings auch elementare Unterschiede beider Wellenformen. Scherwellen sind im Gegensatz zu Ultraschallwellen transversal, ähnlich wie Wasserwellen, die entstehen, wenn ein Gegenstand ins Wasser fällt. Die Wellen breiten sich also senkrecht zum applizierten Impuls aus. Außerdem werden sie im Gewebe schneller abgeschwächt als longitudinale Ultraschallwellen. Zudem erreichen Scherwellen eine viel geringere Ausbreitungsgeschwindigkeit. Wegen der unterschiedlichen Eigenschaften beider Wellenformen können Ultraschallwellen genutzt werden, um durch Scherwellen ausgelöste minimale Gewebsbewegungen zu detektieren und Elastogramme zu erstellen (Cosgrove et al. 2013).

\subsubsection{Transiente Elastographie (TE)}

Die TE misst die Geschwindigkeit einer Scherwelle, die durch einen mechanischen Impuls in Form von Vibration ausgelöst wird. Eine automatische Bewegung der Ultraschallsonde gibt einen 50-Hz-Impuls an die Körperoberfläche ab, nachdem der Untersucher manuell Druck auf den Schallkopf ausübt. Die ausgelösten Scherwellen breiten sich mit annähernd konstanter Geschwindigkeit axial im Gewebe aus. Mithilfe von konventionellen Ultraschallwellen wird die Ausbreitungsgeschwindigkeit (V) der Scherwelle gemessen. Bei bekannter Dichte $(\rho)$ lässt sich die Elastizität der Leber (E) mittels Youngs Modul berechnen $(E=3$ $\rho V^{2}$ ) (Bamber et al. 2013; Dietrich et al. 2017). Ein Nachteil dieses Verfahrens ist, dass der Untersucher keine visuelle Rückmeldung in Form eines B-Bilds oder Elastogramms erhält, denn die Geräte, welche das Verfahren der TE nutzen, wurden speziell für die Elastographie entwickelt. Aufgrund dessen ist die Durchführung einer konventionellen Ultraschalluntersuchung nicht möglich. Das bedeutet zudem, dass der Untersucher keine visuelle Kontrolle über die Auswahl der zu untersuchenden anatomischen Region hat. Außerdem ist eine Verwendung bei Patienten mit Aszites nicht möglich (Bamber et al. 2013). Zu erwähnen ist jedoch, dass zu diesem Elastographieverfahren aktuell die beste klinische Studienlage vorliegt. Es existieren über 1300 Studien, in denen die einzelnen Fibrosestadien - mit der Histologie als Referenzstandard - definiert wurden. Außerdem findet die TE seit 15 Jahren Anwendung in der klinischen Praxis (Sandrin et al. 2003). Die diagnostische Präzision in der Leberfibrose- und Leberzirrhosediagnostik ist bereits ausführlich untersucht worden. Besonders gut geeignet ist die TE zum Ausschluss einer Zirrhose. Eine Differenzierung zwischen den Fibrosestadien F0 und F1 sowie die Bestätigung einer Zirrhose erwiesen sich als schwierig. Allerdings zeigte sich eine gute diagnostische Präzision in der Differenzierung zwischen einer milden bzw. keiner Fibrose und einer signifikanten Fibrose (Castéra et al. 2005; Talwalkar et al. 2007; Boursier et al. 2008; Castera et al. 2008; Friedrich-Rust et al. 2008; 
Tsochatzis et al. 2011; Frulio und Trillaud 2013). Zudem ist die Intra- und Interbeobachtervergleichbarkeit in mehreren Studien als exzellent beurteilt worden (Fraquelli et al. 2007; Boursier et al. 2008). Aufgrund der Vorteile, welche die TE zu bieten hat, ist sie heute ein Teil der deutschen und auch der europäischen Leitlinie für die Behandlung der chronischer Hepatitis C (Mutimer et al. 2014). Auch in der europäischen Leitlinie für die Behandlung von Patienten mit alkoholbedingter Fettlebererkrankung wird die Elastographie als anerkanntes Verfahren in der Leberfibrosediagnostik erwähnt (Mathurin et al. 2012). Ebenfalls hat die deutsche DGVS Leitlinie die TE in den diagnostischen Algorithmus der nicht-alkoholischen Fettlebererkrankung (NAFLD) implementiert (Roeb et al. 2015).

\subsubsection{Punkt-Scherwellen-Elastographie (p-SWE)}

Da die TE nur in wenigen Kliniken verfügbar war, haben in den letzten Jahren nahezu alle Hersteller von Ultraschallgeräten begonnen, eine Elastographie-Funktion (p-SWE oder 2DSWE) in ihre Geräte zu intergieren. Die p-SWE, die auch als acoustic radiation force impuls quantification (ARFI quantification) bekannt ist, hat ebenfalls in der Diagnostik von Leberpathologien an Bedeutung gewonnen. Über die Ultraschallsonde wird ein akustischer Kurzimpuls (push pulse) an das Gewebe abgegeben. Dies führt zu Mikroverschiebungen in der zu untersuchenden Gewebsregion. Durch diese Gewebsverschiebungen werden Scherwellen generiert, die sich, wie oben erwähnt, senkrecht zum gegebenen Impuls ausbreiten. Die Geschwindigkeit der entstandenen Scherwellen wird mittels Ultraschallwellen registriert, indem die zeitliche Ankunft der Scherwelle an zwei Grenzen einer zuvor bestimmten region of interest (ROI) verglichen wird. Die Ausbreitungsgeschwindigkeit wird in Meter pro Sekunde (m/s) angegeben (Nightingale et al. 2003; Bamber et al. 2013). Wie auch bei der TE wird kein Elastogramm erstellt. Im Gegensatz zu TE ist es allerdings möglich, die ROI unter B-Bild-sonographischer Sicht zu platzieren, denn die p-SWE ist in ein konventionelles Ultraschallgerät integriert. Nachteilig ist jedoch, dass nur eine Messung pro B-Bild erzeugt werden kann, da es sich bei dem scherwellengenerierenden Signal nur um einen einzelnen Impuls handelt. Aufgrund dessen ist das repräsentative Areal, verglichen mit der 2D-SWE, eher klein (Bamber et al. 2013). Die p-SWE hat sich bereits in der Leberfibrosediagnostik etabliert, was anhand mehrerer Studien belegt wurde (Takahashi et al. 2010; Sporea et al. 2012). Bezüglich der diagnostischen Präzision der einzelnen Fibrosestadien ist sie mit der TE vergleichbar (Rizzo et al. 2011). Zudem zeigten einige Studien eine exzellente Intra- und Interbeobachtervergleichbarkeit bei gesunden Probanden und auch bei Patienten mit chronischen Lebererkrankungen (Friedrich-Rust et al. 2009; Guzmán-Aroca et al. 2011; Ferraioli et al. 2014).

\subsubsection{2D-Scherwellen-Elastographie (2D-SWE)}

Die 2D-SWE, welche in der vorliegenden Studie verwendet wurde, besitzt eine ähnliche Funktionsweise wie die p-SWE, wurde aber erweitert durch eine größere ROI. Diese ver- 
größerte ROI wird durch das Senden von mehreren simultanen push pulses an unterschiedliche Lokalisationen erreicht. Jeder einzelne push pulse führt zu einem Ultraschall-EchoBild. Die Ausbreitungsgeschwindigkeit der Scherwellen wird, wie bei der p-SWE mithilfe von Ultraschallwellen detektiert. Dieser Prozess wird für jeden einzelnen push pulse wiederholt. Die Tatsache, dass mehrere Scherwellen simultan generiert werden führt dazu, dass mehrere kleine Scherwellen-Bilder erzeugt werden. Diese Einzelbilder werden daraufhin wie ein Mosaik zusammengesetzt. So entsteht ein großes zweidimensionales Elastogramm. Dieses in Farbskalen kodierte Elastogramm kann einem konventionellen B-Bild überlagert oder separat angezeigt werden. Eine blaue Farbgebung zeigt weiches Gewebe, rote Farbgebung hartes Gewebe (siehe Abbildung 1). Die Angabe der Scherwellengeschwindigkeit erfolgt in $\mathrm{m} / \mathrm{s}$ oder mithilfe des Youngs Modul in kPa. Zusätzlich zu der Darstellung des Elastogramms positioniert der Untersucher Messboxen ( $q$-boxes) an gewünschten Lokalisationen innerhalb der ROI. Dies ermöglicht eine Darstellung statistischer Größen wie Median, Standardabweichung, Interquartilabstand, Minimum und Maximum in kPa (Bamber et al. 2013; Frulio und Trillaud 2013; Dietrich et al. 2017).

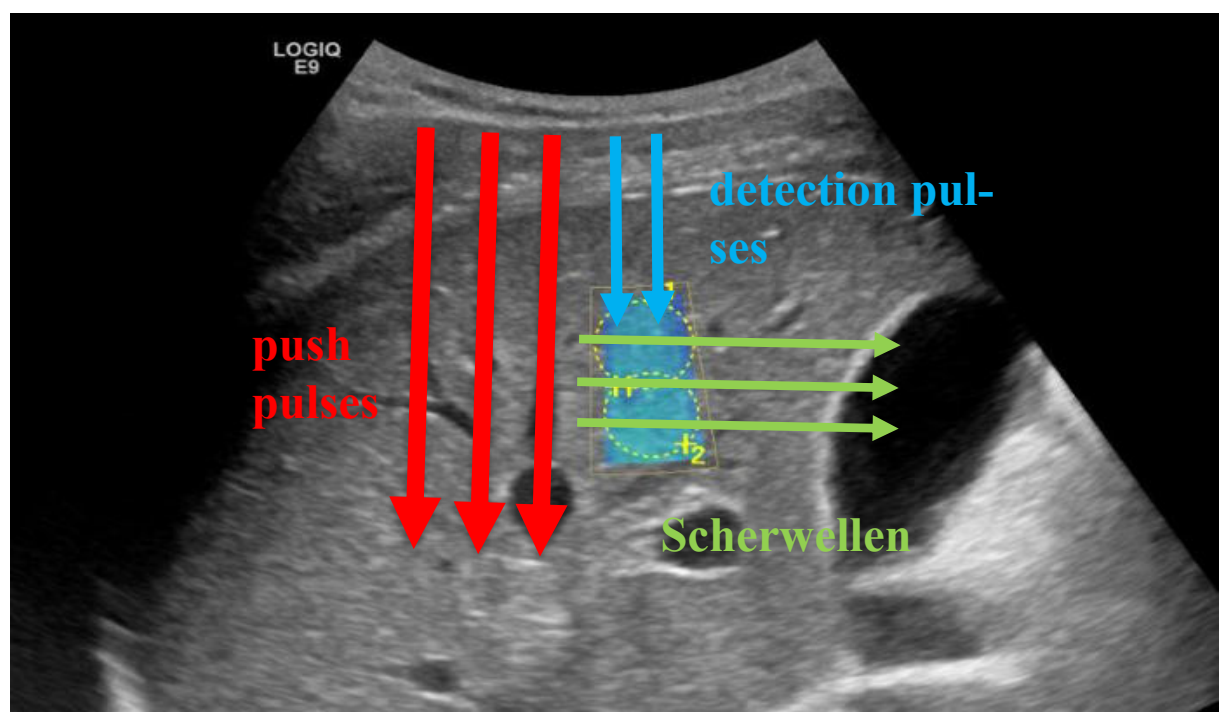

Abbildung 1: Graphische Darstellung der Generierung von Scherwellen mittels push pulses, der Ausbreitung und der Detektion der Scherwellen (Gerät LOGIQ E9, GE).

Die 2D-SWE bietet den Vorteil, zusätzlich zu den statistischen Messwerten, ein farbcodiertes zweidimensionales Echtzeit-Elastogramm der Gewebselastizität anzugeben. Zudem ist die ROI, durch mehrere Messpunkte, deutlich größer und in ihrer Messtiefe und Größe individuell verstellbar. Aufgrund dessen erhält der Untersucher Informationen über ein größeres repräsentatives Gewebsareal als bei den anderen Verfahren. Zwei der hauptvertretenden Firmen, die 2D-SWE in konventionelle Ultraschallgeräte integriert haben sind SuperSonicImaging mit dem Gerät Aixplorer und GE Healthcare mit dem Gerät LOGIQ E9. Aufgrund der Neuheit des Verfahrens (verglichen mit anderen Elastographieverfahren) ist die Studienlage, in Bezug auf die Lebersteifigkeitsmessung (LSM) mittels 2D-SWE, aktuell begrenzt. Auf 
die vorhandene Literatur, besonders zum Gerät LOGIQ E9, welches für diese Studie verwendet wurde, wird im späteren Verlauf eingegangen. Zunächst sollen die Besonderheiten der verwendeten Technik des LOGIQ E9s erläutert werden, die es von anderen Geräten unterscheiden.

Wie oben beschrieben, werden bei der 2D-SWE multiple push pulses simultan an das Gewebe gesendet und somit mehrere Scherwellen erzeugt. Um die Ausbreitungsgeschwindigkeit dieser zu detektieren, werden bis zu 20000 Ultraschallbilder pro Sekunde aufgenommen. Dieses Verfahren nennt sich UltraFast ${ }^{\mathrm{TM}}$ Imaging (Frulio und Trillaud 2013). Eine solch hohe Bildfrequenz ist zeitlich abhängig von zwei Faktoren: der acquisition time (Erfassungszeit) und der cooling time. Die acquisition time ist die Zeit, welche von dem Gerät benötigt wird, um ein Bild zu generieren. Die cooling time die Zeit, bis die nächste Datenerhebung erfolgen kann. Um beide Faktoren zeitlich zu minimieren, nutzt LOGIQ E9 besondere Techniken. Zum einen werden, wie bereits erklärt, multiple push pulses simultan als Muster an das Gewebe gesendet. Dies ermöglicht es, die acquisition time trotz der vielen Impulse nicht zu verlängern. GE nennt dies comb-push excitation. Für die Problematik der hohen Bildfrequenz nutzt das Gerät ein Zeit-Intervall-Tracking-Schema (time-interleaved-shear-wavetracking), wobei die Gewebsantwort an unterschiedlichen Lokalisationen zu jedem Zeitpunkt der Messung detektiert wird. Fehlende Datensätze werden interpoliert. Dies vermindert die Anzahl der benötigten push pulses und reduziert die benötigte Bildfrequenz. Durch dieses Verfahren entsteht eine komplizierte bildliche Darstellung der Scherwellen, die teilweise miteinander kollidieren. Das directional filtering erlaubt Scherwellen nach ihrer Verlaufsrichtung zu filtern, was die Darstellung deutlich vereinfacht. Mittels local shear wave speed estimation wird für beide Verlaufsrichtungen (links und rechts) ein Scherwellenbild und eine Korrelationskoeffizientenkarte generiert. Diese werden dann übereinandergelegt und ein zweidimensionales Elastogramm entsteht (GE Healthcare 2014).

Wie bereits erwähnt, ist die Studienlage bezüglich Leberfibrosediagnostik mittels 2D-SWE limitiert. Ferraioli et al. untersuchten die diagnostische Präzision der Fibrosediagnostik anhand des Aixplorers (SuperSonicImaging). Hierbei wurde deutlich, dass die 2D-SWE, verglichen mit TE (Gerät Fibroscan), über eine größere Sensitivität in der Diagnostik einer signifikanten (METAVIR F $\geq 2$ ) und einer fortschreitenden Fibrose (METAVIR F = 4) verfügt (Ferraioli et al. 2012). Dies wurde auch anhand weiterer Studien, die Ergebnisse von Leberbiopsien und von Messungen mit der 2D-SWE miteinander verglichen, bestätigt (Leung et al. 2013). Im Jahr 2016 wurde eine Meta-Analyse aller bis dahin zu 2D-SWE vorhandenen Literatur veröffentlicht. Es wurde gezeigt, dass die 2D-SWE eine gute Methode zur Beurteilung des Schweregrads der Leberfibrose darstellt (Jiang et al. 2016).

Das für diese Studie verwendete Gerät LOGIQ E9 (GE Healthcare) wurde im Hinblick auf die diagnostische Genauigkeit von Leberfibrose bei 174 Patienten mit chronischen Lebererkrankungen untersucht. Als Referenz galt hier die Leberbiopsie. Eine signifikant positive 
Korrelation mit den histologischen Fibrosestadien nach METAVIR wurde beobachtet. AuBerdem ergab sich bei der Kontrollgruppe gesunder Probanden eine exzellente Interbeobachtervergleichbarkeit (Serra et al. 2018). Ein Vergleich der Interbeobachtervariabilität an Geräten der p-SWE, der TE und dem LOGIQ E9 zeigte vergleichbar gute Ergebnisse. Besonders das LOGIQ E9 erzielte hier eine exzellente Reproduzierbarkeit. Die Messungen wurden allerdings nicht an Probanden/Patienten, sondern an einem Leberfibrosephantom durchgeführt. Die exakten Messwerte in $\mathrm{kPa}$ variierten teilweise jedoch deutlich zwischen den Geräten, trotz der Verwendung identischer Phantome (Mulabecirovic et al. 2018). Moga et al. untersuchten die Inter- und Intrabeobachtervariabilität an lebergesunden Probanden und Patienten mit Leberzirrhose. Sie verglichen die Werte der LSM von drei Untersuchern mit jeweils unterschiedlichem Erfahrungsstand in der Sonographie und in der Elastographie. Die Ergebnisse sowohl der Inter- als auch der Intrabeobachtervergleichbarkeit erwiesen sich als exzellent (Moga et al. 2018).

Bende et al. veröffentlichten zwei Artikel mit teilweise konträren Aussagen. In einer der beiden Studien wurde eine starke positive Korrelation zwischen Ergebnissen der TE und der 2D-SWE (mit LOGIQ E9), sowohl bei Patienten mit chronischen Lebererkrankungen als auch bei gesunden Probanden, beschrieben. Allerdings waren die Messwerte der 2D-SWE bei Probanden mit Fibrose im Mittel signifikant niedriger als die der TE (Bende et al. 2017). Eine zweite Studie mit ausschließlich lebergesunden Probanden zeigte nur eine schwache positive Korrelation beider Verfahren. Zudem waren die Messwerte der 2D-SWE bei lebergesunden Probanden höher als die Messwerte der TE (Bende et al. 2018). Fang et al. stellte die 2D-SWE (Gerät LOGIQ E9 GE) der p-SWE (Gerät VirtualTouch Siemens) gegenüber. Hier zeigte sich eine signifikante Korrelation der Messwerte beider Verfahren bei gesunden Probanden (Fang et al. 2017). Weitere Studien werden erwartet.

Wie anhand der oben erwähnten Arbeiten bereits deutlich wurde, sind die Messwerte der Elastographiegeräte und auch der einzelnen Verfahren untereinander nicht direkt vergleichbar. Dies liegt unter anderem daran, dass unterschiedliche Techniken und Berechnungsmethoden verwendet werden. Deshalb existieren systemspezifische Cut-off-Werte. Die erhobenen Messwerte sollten daher immer in Abhängigkeit vom verwendeten Gerät und Verfahrens interpretiert werden (Goertz 2015; Piscaglia et al. 2016).

\subsection{Einflussfaktoren der Scherwellen-Elastographie}

Neben der Fibrose existieren weitere physiologische und pathologische Faktoren, die die Steifigkeit der Leber beeinflussen können. Der Großteil der dazu vorhandenen Literatur bezieht sich auf Studien mit der TE. Es ist aber davon auszugehen, dass die untersuchten Einflussfaktoren auch für die anderen Verfahren von Bedeutung sind (Dietrich et al. 2017). In dem Großteil der durchgeführten Studien wurde ein geschlechtsspezifischer Unterschied zwischen der LS weiblicher und männlicher Probanden beobachtet. Männer zeigten hier 
durchschnittlich höhere LS als Frauen (Colombo et al. 2011; Ling et al. 2013; Huang et al. 2014; Liao et al. 2015). Nur Horster et al. und Madhok et al. beschrieben keine signifikanten Unterschiede bezüglich des Geschlechts (Horster et al. 2010; Madhok et al. 2013). Auch der Einfluss des Alters und des body mass index (BMI) wurde untersucht. Das Alter zeigte keinen Effekt auf die LS (Kim et al. 2010; Popescu et al. 2011; Huang et al. 2014). Auch bezüglich des BMIs ergab sich, abgesehen von einer Studie (Liao et al. 2015), keine signifikante Beeinflussung der LS (Kim et al. 2010; Popescu et al. 2011; Son et al. 2012; Sirli et al. 2013; Huang et al. 2014). Anhand mehreren Studien wurde gezeigt, dass die LS mit hepatischer Inflammation ansteigt (Arena et al. 2008; Sagir et al. 2008). Zudem führt ein Anstieg der Serumtransaminasen ebenfalls zu einem Anstieg der LS (Coco et al. 2007; Arena et al. 2008). Neben den genannten Faktoren haben auch die extrahepatische Cholestase (Millonig et al. 2008), die Einnahme von Beta-Blockern (Reiberger et al. 2012) und alkoholbedingte Lebererkrankungen eine Erhöhung der LS zur Folge (Mueller et al. 2010; Bardou-Jacquet et al. 2013). Auch Messungen direkt im Anschluss an körperliche Aktivitäten führen zu einem Anstieg der Messwerte, weswegen Patienten mindestens 10 min vor der Untersuchung ruhen sollten (Gersak et al. 2016b). Außerdem wurde gezeigt, dass den Blutfluss beeinflussende Faktoren ebenfalls die LS verändern. Dazu zählen ein erhöhter ZVD (zentral venöser Druck) (Millonig et al. 2010) und die akut dekompensierte Herzinsuffizienz (Colli et al. 2010). Unklar ist der Einfluss einer Steatose auf die LSM. Es existieren sowohl Studien die dies als Einflussfaktor darstellten (Macaluso et al. 2014; Petta et al. 2015) als auch Studien die das Gegenteil zeigten (Yoneda et al. 2008; Wong et al. 2010). In einer Arbeit zur 2D-SWE wurde der Einfluss einer milden Steatose (<33\% Verfettung) auf die LSM ausgeschlossen (Suh et al. 2014). Ein weiterer wichtiger Einflussfaktor ist die Nahrungsaufnahme. Hierauf soll im Verlauf genauer eingegangen werden.

Zusammenfassend lässt sich feststellen, dass die bekannten Einflussfaktoren bei der Deutung der Messwerte immer zu berücksichtigen sind. Die Interpretation der erhobenen Lebersteifigkeitswerte sollte ausschließlich durch einen Experten und unter Berücksichtigung der jeweiligen klinischen Fragestellung bzw. der Ätiologie der Erkrankung erfolgen (Dietrich et al. 2017).

\subsubsection{Nahrungsaufnahme und Scherwellen-Elastographie}

Die Analyse der Nahrungsaufnahme als Einflussfaktor auf die LS ist zentraler Gegenstand meiner Arbeit. Im Detail möchte ich untersuchen, ob die Nahrungsaufnahme Einfluss auf die LSM mittels 2D-SWE, bei gesunden Probanden hat. Welche Relevanz die Klärung dieser Frage hat, soll im Folgenden verdeutlicht werden.

Zunächst soll untersucht werden, ob die Nahrungsaufnahme grundsätzlich eine Auswirkung auf die LS hat, denn dies hätte die Konsequenz, dass Patienten zu einer elastographischen Untersuchung nüchtern einbestellt werden sollten. Im Alltag einer Klinik sind zwei Patientengruppen zu unterscheiden, die ambulanten und die stationären Patienten. Elektiv geplante 
sonographische Untersuchungen werden, wie auch andere diagnostische Verfahren im klinischen Alltag, möglichst am Vormittag oder frühen Nachmittag durchgeführt. Aufgrund von dringenden Notfällen oder aus Kapazitätsgründen kann es zu Verzögerungen oder Terminverlegungen kommen. Dies bedeutet für Patienten, die nach einer nächtlichen Fastenperiode einbestellt wurden, weiter auf Nahrung zu verzichten. Das führt zu Unannehmlichkeiten für die Patienten. Es stellt sich die Frage, für welchen Zeitraum es vertretbar ist, einen Patienten nüchtern zu lassen. Zum anderen stellt die verlängerte Nüchternperiode ein Problem für den Stationsalltag dar, denn dem Pflegepersonal ist es nicht möglich, die Patienten zu kontrollieren. Auch bei ambulanten Patienten gibt es Unsicherheiten bezüglich der Nüchternheit. Viele dieser Patienten können den Zeitraum, in dem sie auf Nahrung verzichtet haben, nicht genau benennen. Hier stellt sich die Frage, ob es einen relevanten diagnostischen Unterschied zwischen verschiedenen Nüchternzeiträumen gibt. Wäre dieser zeitliche Unterschied nicht relevant, würde das den klinischen Alltag erleichtern. Eine Nahrungskarenz von wenigen Stunden ist im Vergleich zu einer nächtlichen Fastenperiode leichter umsetzbar. Falls die Fastenperiode nicht bekannt ist, besteht eine Unsicherheit bei der Interpretation der erhobenen Werte. Stellt ein erhöhter Messwert einen Hinweis auf eine Leberfibrose dar oder ist der Messwert einer zeitlich verringerten Fastenperiode geschuldet? Wie wäre der Nüchternwert des Patienten? Wie bereits erwähnt hat die Elastographie bereits Einzug in die Leitlinien für die Behandlung chronischer Hepatitis $\mathrm{C}$ und NAFLD gefunden. Besonders hier ist es wichtig, ob die Werte des Patienten intraindividuell, bei unterschiedlich langen Fastenperioden, vergleichbar sind. Dies ist von besonderer Bedeutung, denn die Messwerte der Elastographie haben hier direkten Einfluss auf das Management der Erkrankung. Schlussendlich stellt sich die Frage, welche Konsequenz aus auffälligen Messungen, nach unbekannter oder zu kurzer Fastenperiode, resultiert. Eine nochmalige Einbestellung des Patienten oder eine weiterführende invasive Diagnostik stellen einerseits einen möglicherweise vermeidbaren Kosten- und Zeitaufwand für die Klinik dar. Andererseits ist die Wiedervorstellung mit einer großen Unsicherheit und Sorge des Patienten verbunden. Zudem sind Komplikationen durch weitere invasive diagnostische Verfahren, wie zum Beispiel die Leberbiopsie, nie auszuschließen. All diese Argumente machen die Wichtigkeit der Analyse des Einflusses der Nahrungsaufnahme auf die LSM deutlich.

Einige Arbeitsgruppen haben bereits Artikel zu dieser Thematik veröffentlicht. Anhand der aktuellen Studienlage ist zu erkennen, dass die Nahrungsaufnahme einen signifikanten Anstieg der LS zur Folge zu haben scheint. Allerdings zeigten sich im Vergleich der Studien teilweise starke Diskrepanzen in den Lebersteifigkeitsveränderungen. Es wurden postprandial Anstiege der LS von 2,2 \% bis hin zu $31 \%$ beobachtet (Goertz et al. 2012; Popescu et al. 2013; Gersak et al. 2016a; Gersak et al. 2016b; Kjærgaard et al. 2017). Es herrscht Uneinigkeit bezüglich der empfohlenen Fastenperiode vor der Durchführung einer Elastographie, da verschiedene Zeiträume bis zum Erreichen des Ausgangswerts beschrieben wurden. Einige Arbeiten empfehlen eine nächtliche Fastenperiode (Berzigotti et al. 2013), andere eine 
Nüchternheit von 3 h (Mederacke et al. 2009; Popescu et al. 2013; Lemoine et al. 2014) und wiederum andere Arbeiten kamen zu dem Ergebnis, dass $2 \mathrm{~h}$ ausreichend seien (Goertz et al. 2012; Arena et al. 2013; Alvarez et al. 2015). Außerdem wurde die Relevanz von unterschiedlich langen Nüchterzeiträumen vor der LSM bislang nicht untersucht. Auch der Einfluss von Faktoren wie Geschlecht, BMI und Alter, in Bezug auf den Anstieg der LS nach Nahrungsaufnahme, ist nicht eindeutig geklärt. Ein postprandialer Anstieg des portalen Blutflusses wurde in der Mehrzahl der Arbeiten gezeigt, allerdings korrelierte dieser nicht signifikant mit dem Anstieg der LS (Berzigotti et al. 2013; Alvarez et al. 2015). Unklarheit herrscht bezüglich des Einflusses der Nahrungsaufnahme auf die Validität der LSM. Es besteht der Verdacht, dass die Einnahme einer Mahlzeit die Frequenz von validen Messungen reduziert (Lemoine et al. 2014). Wie auch die anderen Einflussfaktoren betreffend, bezieht sich der Großteil der vorhandenen Literatur zum Thema Nahrungsaufnahme auf Beobachtungen der TE. Die Studienlage zur 2D-SWE und vor allem zu dem hier verwendeten Gerät (LOGIQ E9) ist aktuell begrenzt. Wie bereits oben erläutert, können die Elastographiegeräte und auch die einzelnen Verfahren untereinander nicht direkt verglichen werden. Aufgrund dessen ist es von Relevanz den Einfluss der Nahrungsaufnahme auf die LS, gemessen mit dem Gerät LOGIQ E9, zu untersuchen. Darüber hinaus zeigte die einzige Studie zu LOGIQ E9, im Vergleich zu allen anderen bisher veröffentlichten Studien, konträre Ergebnisse. Diese kleine, bisher nur als Abstract publizierte Studie, beobachtete keinen signifikanten Einfluss der Nahrungsaufnahme auf die LS (Popescu et al. 2016). Als mögliche Ursache für einen geringeren Einfluss der Nahrungsaufnahme bei dem Gerät LOGIQ E9 könnte vermutet werden, dass die LS-Werte bei gesunden Probanden vergleichbar oder sogar höher sind als Messungen der TE (Bende et al. 2018), Patienten mit höhergradiger Fibrose jedoch deutlich niedrigere Werte aufweisen (Bende et al. 2017; Barr 2018). Wenn eine Zunahme der Fibrose nur eine geringe Erhöhung der LS bewirkt, könnte der Einfluss der Nahrungsaufnahme vernachlässigbar sein.

Das Ziel der vorliegenden Arbeit ist die Frage zu beantworten, ob die Nahrungsaufnahme einen Einfluss auf die 2D-SWE bei lebergesunden Probanden hat. Außerdem soll untersucht werden, inwiefern Faktoren wie Geschlecht, BMI, Alter, Alkoholkonsum, Medikamenteneinnahme und die Nüchternperiode sowohl die LS vor Einnahme einer Mahlzeit als auch die mögliche nahrungsbedingte Veränderung der LS beeinflussen. Zusätzlich wird geprüft, ob die Nahrungsaufnahme die Validität der LSM verändert und ob die erhobenen Messwerte der LS mit denen des PBF und des GBV korrelieren. 


\section{Material und Methoden}

\subsection{Studiendesign und Patientenkollektiv}

Bei der durchgeführten Studie handelt es sich um eine prospektive, monozentrische klinische Studie zur Untersuchung des Einflusses der Nahrungsaufnahme auf die LS gesunder Probanden, durchgeführt in der Klinik für Gastroenterologie und gastrointestinale Onkologie des Universitätsklinikums Göttingen. Eine positive Begutachtung der Ethikkommission der Universitätsmedizin Göttingen lag am 31.07.2017 mit dem Aktenzeichen 5/7/17 vor.

Es wurden 100 freiwillige Probanden in dem Zeitraum vom 16.08.2017 bis 31.11.2017 untersucht. Die Rekrutierung der 100 Probanden erfolgte über direkte Ansprache.

Voraussetzung für die Teilnahme an der Studie waren Einwilligungsfähigkeit, Volljährigkeit und die freiwillige Teilnahme. Außerdem mussten die Probanden mindestens $3 \mathrm{~h}$ vor der ersten Untersuchung auf jegliche Nahrungs- und Flüssigkeitsaufnahme verzichten. Zusätzlich war es erforderlich, dass es dem Studienteilnehmer möglich war, die Mahlzeit innerhalb von maximal 10 min zu sich zu nehmen. Probanden mit Schluckstörungen, Unverträglichkeiten oder Allergien gegen Bestandteile der Nahrung wurden von der Studie ausgeschlossen. Auch Probanden mit Diabetes mellitus Typ 1 oder Typ 2 wurden nicht mit einbezogen. Vor der Untersuchung wurden die Studienteilnehmer über die nachfolgenden Messungen aufgeklärt und willigten schriftlich ein (Einwilligungserklärung siehe Anhang Abbildung 16). Des Weiteren wurde ein Fragebogen ausgefüllt, der folgende Daten erfasste: Geschlecht, Geburtsdatum, Größe in cm, Gewicht in kg, vorhandene Lebererkrankungen, Herzinsuffizienz, Medikamenteneinnahme, Alkoholkonsum (0 g/Tag, 0 - 10 g/Tag, 10 - 20 g/Tag, 20 - 30 g/Tag, > 30 g/Tag) und Stunden der Nüchternheit ( 3 h, 4 h, 5 h, 6 h, 7 h, 8 h, 9 h, 10 h, > 10 h). Ergänzt wurde der Fragebogen durch den Untersucher mit einer Probandennummer, dem Messdatum, der Messuhrzeit und dem BMI in kg/m² (Fragebogen siehe Anhang Abbildung 15).

\subsection{Untersuchungsablauf}

Durchgeführt wurde die folgende Untersuchung mit dem Konvexschallkopf C1-6 (4 MHz) des Geräts LOGIQ E9 der Firma GE Healthcare mit der Elastographie Software R1.0.6. Das verwendete Gerät nutzt das Prinzip der 2D-SWE.

Der Untersuchungsablauf bestand aus präprandialen Messungen, der Nahrungsaufnahme, einer Verdauungsphase und postprandialen Messungen. Das exakte Vorgehen wird im Folgenden beschrieben und in Abbildung 4 dargestellt.

Nach einer intensiven Einarbeitung in die Sonographie und supervidierten Elastographien an über 50 Personen habe ich alle 200 Messungen selbstständig durchgeführt. 
Bezüglich der LSM wurde sich an den Empfehlungen des Herstellers sowie der aktuellen EFSUMB-Leitlinie und Empfehlungen zur klinischen Anwendung der Leberelastographie orientiert (Bende et al. 2017; Barr 2018).

Zunächst folgte eine körperliche Ruhephase von 10 - 20 min vor Untersuchungsbeginn. Anschließend lag der Proband, der seit mindestens 3 h keine Nahrung oder Flüssigkeit zu sich genommen hatte, mit entkleidetem Oberkörper und extendiertem rechten Arm in Rückenlage auf der Untersuchungsliege. Daraufhin verschaffte sich der Untersucher mithilfe der BMode-Sonographie einen Überblick über die Anatomie des Abdomens. Der Studienteilnehmer befand sich in Atemmittellage. Auffälligkeiten (Leberparenchymveränderungen, Hämangiome, Cholezystolithiasis etc.) wurden dokumentiert. Der craniocaudale Längsdurchmesser der Leber wurde in der rechten Medioclavicularlinie vermessen.

Im Anschluss folgte die präprandiale LSM. Die Ultraschallsonde wurde in den Intercostalräumen (ICR) so platziert, dass der rechte Leberlappen einsehbar war. Bei der Wahl der ROI war darauf zu achten, dass diese frei von Gefäßen, minimal $1-2 \mathrm{~cm}$ und maximal $6 \mathrm{~cm}$ unterhalb der Leberkapsel positioniert wurde (Dietrich et al. 2017) (Beispielbild siehe Abbildung 2).

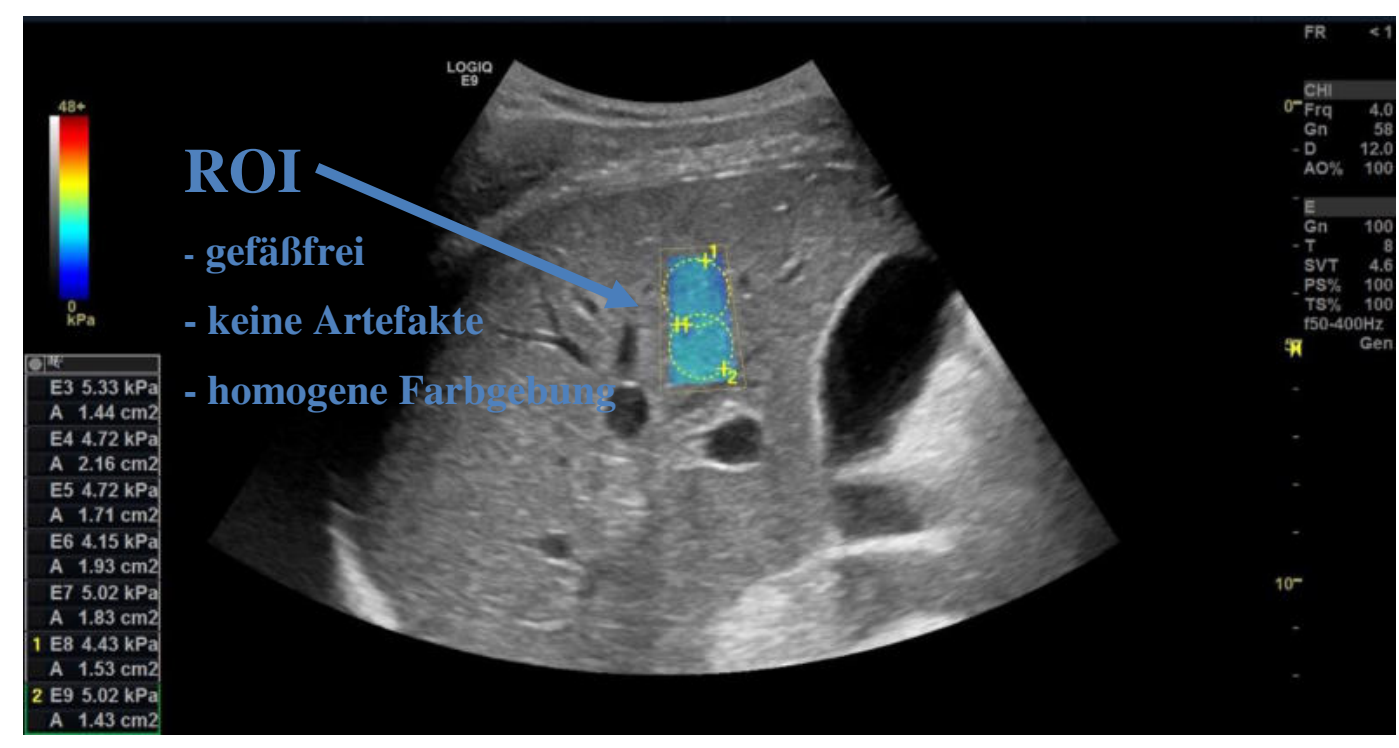

Abbildung 2: Beispielbild der LSM mittels 2D-SWE (Gerät LOGIQ E9, GE).

Der Proband wurde gebeten, das Atmen in Atemmittellage für einige Sekunden einzustellen, um mögliche Störvariablen wie Atembewegungen auszuschalten. Die ersten Analyseboxen wurden durch die Betätigung des Bedienungsfelds generiert. Nach Abschluss der Messung atmete der Patient weiter. Da seitens des Herstellers eindeutige Qualitätskriterien bezüglich der Auswahl verwertbarer ROIs fehlten, orientierte sich der Untersucher bei der Auswahl dieser an folgenden Empfehlungen anderer Arbeiten, die sich mit 2D-SWE befasst haben. Messungen in den farbkodierten ROIs wurden nur dann durchgeführt, wenn mehr als zwei Drittel der ROI homogen gefärbt und die Farbübergänge fließend waren. Außerdem wurden nur ROIs verwendet, in denen Artefakte (Verpixelungen, Fehlen eines Signals) weniger als 
ein Drittel des Bildes einnahmen und keine scharfen Farbübergänge von blauen zu roten Arealen existierten (Grgurević et al. 2015). ROIs, die diesen Kriterien nicht entsprachen, wurden nicht verwendet (Abbildung 3).

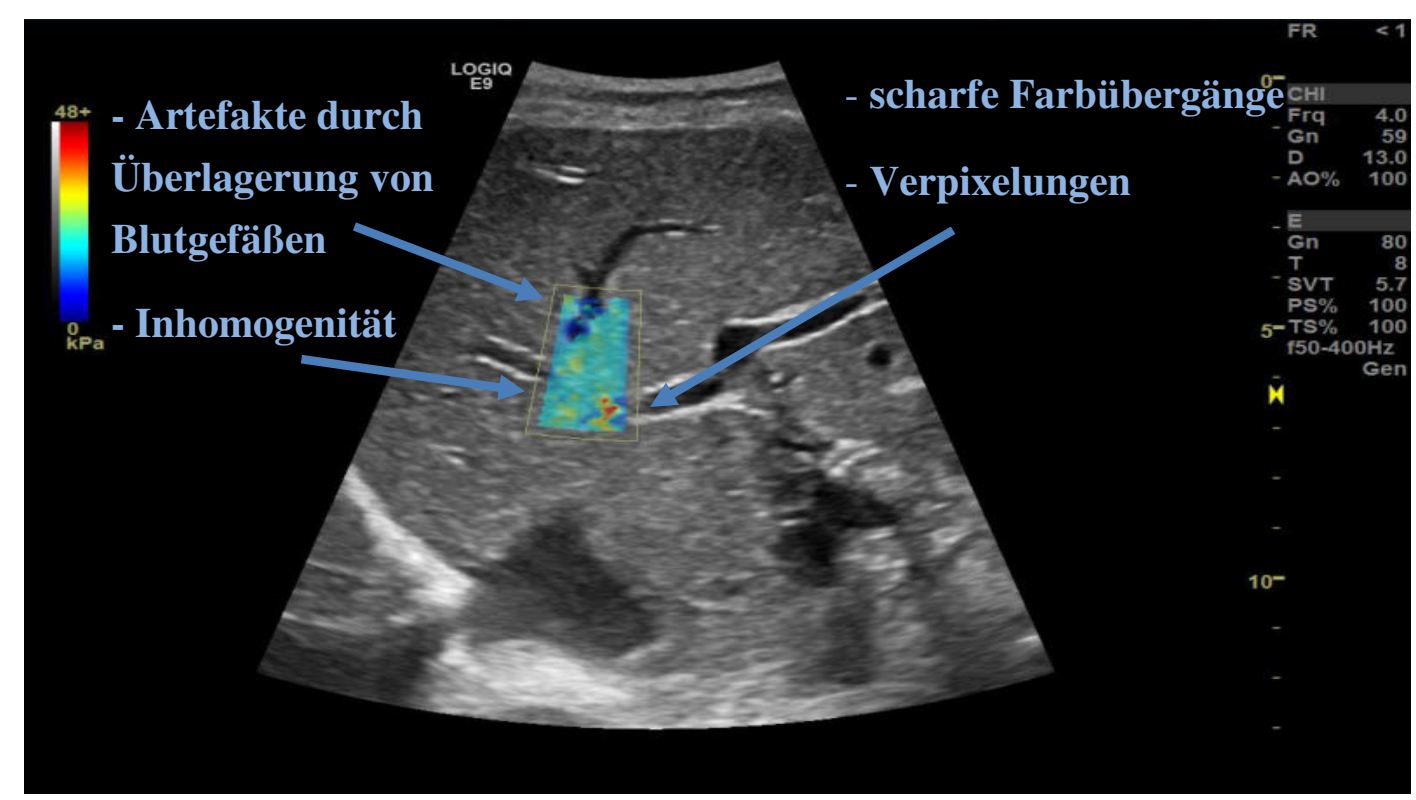

Abbildung 3: Beispielbild einer nicht den Qualitätskriterien entsprechenden ROI (Gerät LOGIQ E9, GE).

Nach Auswahl einer den Qualitätskriterien entsprechenden ROI wurden kreisförmige Messboxen (q-boxes), (Durchmesser mindestens $1 \mathrm{~cm}$ ) innerhalb der ROIs ausgewählt, wobei Überlappungen vermieden wurden. An diesen Regionen berechnete das Gerät nun die LS in $\mathrm{kPa}$. Dieses Vorgehen wurde so lange wiederholt, bis 12 voneinander unabhängige Einzelmessungen generiert worden waren. Die LSM wurden als Zahlenwert in $\mathrm{kPa}$ automatisch in einem Arbeitsblatt des Geräts gespeichert. Auf diesem wurde am Ende der Messung neben den Einzelwerten auch der Median in $\mathrm{kPa}$ und der Interquartilsabstand (IQR) in $\mathrm{kPa}$ angegeben. Gemäß den Qualitätskriterien des Herstellers wurden nur Messungen mit einem IQRWert $<30 \%$ des Medians als valide anerkannt. Die Dauer der Elastographie-Messung betrug in der Regel weniger als 5 min.

Darüber hinaus wurde der Pfortaderblutfluss (PBF) in Zentimeter pro Sekunde $(\mathrm{cm} / \mathrm{sec})$, der Gallenblasenlängsdurchmesser in Zentimeter $(\mathrm{cm})$, die Gallenblasenwanddicke in Millimeter (mm) und das Gallenblasenvolumen (GBV) in Milliliter (ml) ermittelt. Die Vermessung der Gallenblase erfolgte von subcostal in zwei Ebenen. Der PBF wurde von intercostal kurz vor der Aufzweigung in rechten und linken Hauptast gemessen.

Zudem wurde die Echogenität der Leber und der rechten Niere im Hinblick auf Anzeichen einer Steatose, miteinander verglichen. Dies ist von Relevanz, da der Einfluss der Steatose auf die Elastographie wissenschaftlich bisher noch unklar ist (Dietrich et al. 2017). 
Nach den abgeschlossenen präprandialen Messungen folgte die orale Aufnahme von zwei Fresubin-Trinkflaschen mit insgesamt $800 \mathrm{kcal}$ in $400 \mathrm{ml}(2 \mathrm{kcal} / \mathrm{ml} ; 10 \mathrm{~g} / 100 \mathrm{ml}$ Proteine; 7,8 g/100 ml Fett; 22,5 g/100 ml Kohlenhydrate). Zur Auswahl standen zwei Geschmacksrichtungen. Der Proband nahm diese Flüssigmahlzeit in maximal $10 \mathrm{~min}$ zu sich. Nach vollständigem Verzehr wurde vor Beginn des zweiten Untersuchungsablaufs 30 bis 40 min abgewartet.

Im Anschluss folgte die postprandiale Untersuchung, in der alle Parameter (außer der Leberlängsdurchmesser) nach dem gleichen Vorgehen nochmals erhoben wurden. Zuvor beobachtete anatomische Leitstrukturen in räumlicher Nähe der ROI wurden dafür genutzt, alle Messungen an der möglichst selben Stelle durchzuführen. Zur Veranschaulichung des gesamten Untersuchungsablaufs siehe Abbildung 4.

Alle gemessenen und erhobenen Daten wurden pseudonymisiert handschriftlich und elektronisch gespeichert und im Anschluss in einer Microsoft-Excel-Tabelle dokumentiert. Insgesamt nahmen der gesamte Untersuchungsablauf, die Verdauungsperiode und die anschlieBende Dokumentation pro Proband ungefähr 60 min in Anspruch.

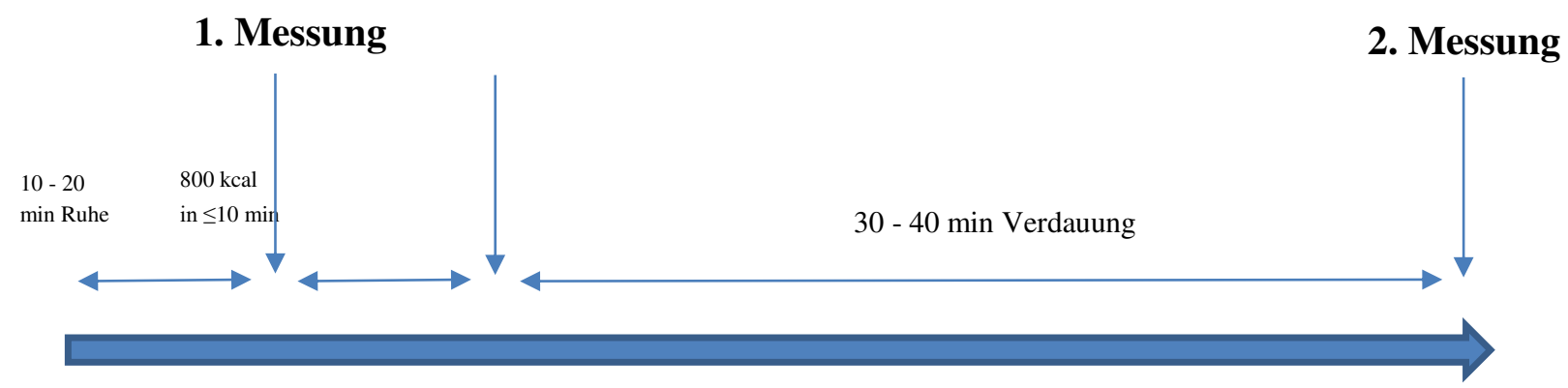

Abbildung 4: graphische Darstellung des Untersuchungsablaufs.

Die statistische Auswertung wurde mithilfe des Programms Statistica (TIBCO, Version 13.3) durchgeführt. Die deskriptive Darstellung der erhobenen Daten erfolgte mittels Angabe des Mittelwerts, der Standardabweichung und des Minimums und Maximums. Das Signifikanzniveau wurde wie üblich auf $\mathrm{p}<0,05$ festgelegt. Zunächst wurden die Daten, mittels graphischer Darstellung als Histogramm, auf Normalverteilung geprüft. Der t-Test für abhängige Stichproben wurde genutzt, um die Mittelwerte der Messungen, welche vor und nach Nahrungsaufnahme durchgeführt wurden, miteinander zu vergleichen. Darunter befanden sich die Erhebung der LS, des IQR-Werts, des PBF und des GBV. Geschlechtsspezifische Mittelwertunterschiede wurden mithilfe des t-Tests für unabhängige Stichproben durchgeführt, ebenso die Unterschiede bezüglich Probanden mit und ohne Medikamenteneinnahme. Varianzanalysen (ANOVA) wurden genutzt, um die Mittelwerte der verschiedenen Alkoholkonsumgruppen und Nüchterngruppen zu vergleichen. Zur Untersuchung von 
Abhängigkeiten zwischen den normalverteilten, metrisch skalierten Variablen (LS, LS-Ausgangswert, IQR, PBF, GBV, BMI, Nüchternperiode) wurden Korrelationen nach Pearson und lineare Regressionsanalysen durchgeführt. Unter anderem wurde somit der Einfluss des LS-Ausgangswerts auf den postprandialen Lebersteifigkeitsanstieg (LSA), die Korrelation der LS und des PBF und die Korrelation der postprandialen Veränderungen beider Parameter analysiert. Da das Alter der Probanden keine Normalverteilung zeigte, wurde hier eine Korrelation nach Spearman verwendet. Um die praktische Relevanz der Ergebnisse zu verdeutlichen, diente die Einteilung der Effektstärke nach Cohen (1992) als Orientierung. Sowohl für die Korrelationen nach Pearson als auch für die Korrelationen nach Spearman galt $\mathrm{r}=$ 0,10 als ein schwacher, $r=0,30$ als ein mittlerer und $r=0,50$ als ein starker Effekt $(r=$ Korrelationskoeffizient). 


\section{Ergebnisse}

\subsection{Demographie des Probandenkollektivs}

Insgesamt wurden 100 Probanden untersucht. Die prä- und postprandialen Messungen waren, gemäß den oben genannten Qualitätskriterien in $100 \%$ der Fälle erfolgreich. Unter den 100 Probanden befanden sich 52 Frauen und 48 Männern. Das mittlere Alter des gesamten Kollektivs lag bei 25,8 ( \pm 6,1) Jahren. Die Altersspanne der Frauen lag bei 19 - 54 Jahren, die der Männer bei 19 - 55 Jahren. Bei den Frauen entsprach das durchschnittliche Alter 25,2 ( \pm 4,7) Jahren, bei den Männer lag dies bei 26,4 ( \pm 7,2) Jahren.

Ein weiteres zu untersuchendes Kriterium stellte der BMI dar, der nach der folgenden Formel berechnet wurde:

$\mathrm{BMI}=$ Körpergewicht $[\mathrm{kg}] /$ Körpergröße $[\mathrm{m}]^{2}$.

Der mittlere BMI aller Studienteilnehmer lag bei 22,43 $( \pm 2,37) \mathrm{kg} / \mathrm{m}^{2}$. Die Spannweite der Frauen betrug $17,26 \mathrm{~kg} / \mathrm{m}^{2}-27,73 \mathrm{~kg} / \mathrm{m}^{2}$, mit einem durchschnittlichen BMI von 21,43 ( \pm $1,91) \mathrm{kg} / \mathrm{m}^{2}$. Die der Männer $19,50 \mathrm{~kg} / \mathrm{m}^{2}-30,76 \mathrm{~kg} / \mathrm{m}^{2}$, mit einem durchschnittlichen BMI von $23,52( \pm 2,33) \mathrm{kg} / \mathrm{m}^{2}$.

Ein Teilnehmer zeigte sonographische Anzeichen einer Steatose. Laut medizinischer Vorgeschichte litt keiner der Studienteilnehmer an Lebererkrankungen oder einer Herzinsuffizienz.

Insgesamt gaben 30 Probanden an, regelmäßig Medikamente einzunehmen, darunter 26 Frauen und 4 Männer. Von den weiblichen Probandinnen nahmen 23 ein orales Kontrazeptivum ein. Je eine Teilnehmerin dieser Gruppe nahm neben dem oralen Kontrazeptivum noch L-Thyroxin, SSNRI (Venlafaxin) oder ein inhalatives Kortisonpräparat ein. Die Verhütung mittels Nuvaring (Etonogestrel/Ethinylestradiol) wurde von einer Probandin angegeben. Eine Probandin nahm regelmäßig Mesavancol ein. Amoxicillin wurde von einer Teilnehmerin eingenommen (3 Tage). Unter den Männern nahmen zwei Probanden L-Thyroxin (50 $\mu \mathrm{g}$ bzw. $200 \mu \mathrm{g}$ ) ein. Ein Teilnehmer gab die Einnahme von Ramipril 5 mg an. Ein weiterer männlicher Proband hatte regelmäßig ein xylomethazolinhaltiges Nasenspray angewendet.

Die Angabe des Alkoholkonsums wurde in fünf Gruppen unterteilt, die den Alkoholkonsum pro Tag definierten $(0 \mathrm{~g} / \mathrm{d}, 0-10 \mathrm{~g} / \mathrm{d}, 10-20 \mathrm{~g} / \mathrm{d}, 20-30 \mathrm{~g} / \mathrm{d},>30 \mathrm{~g} / \mathrm{d})$.

Die Gruppe mit 0 g/d umfasste 26 Probanden (15 Frauen, 11 Männer). 50 Teilnehmer gaben 0 - $10 \mathrm{~g} / \mathrm{d}$ an (29 Frauen, 21 Männer). Einen Alkoholkonsum von 10 - $20 \mathrm{~g} / \mathrm{d}$ gaben 20 Probanden (8 Frauen, 12 Männer) an. Die Gruppe mit einem Alkoholkonsum von 20 - 30 $\mathrm{g} / \mathrm{d}$ beinhaltete 4 Probanden (0 Frauen, 4 Männer). Einen Alkoholkonsum von > 30 g/d gab keiner der Teilnehmer an (Tabelle 1). 
Zudem wurden von jedem Probanden die Stunden der Nüchternheit vor Untersuchungsbeginn angegeben $(3 \mathrm{~h}$ bis $>10 \mathrm{~h})$. Die Probanden hatten im Durchschnitt 5,5 $( \pm 3,1) \mathrm{h}$ ohne Nahrungsaufnahme verbracht. Die Frauen waren im Mittel seit 5,3 $( \pm 3,0)$ h nüchtern. Die Männer hatten im Durchschnitt seit 5,6 $( \pm 3,3)$ h keine Nahrung zu sich genommen. Seit $3 \mathrm{~h}$ nüchtern waren insgesamt 32 Probanden (15 Frauen, 17 Männer). $4 \mathrm{~h}$ auf Nahrung verzichtet hatten 34 Probanden (21 Frauen, 13 Männer). 5 h keine Nahrung zu sich genommen hatten 5 Probanden ( 3 Frauen, 2 Männer). Seit 6 h nüchtern waren 5 Studienteilnehmer (2 Frauen, 3 Männer). 7 h Nüchternheit gab keiner der Probanden an. 8 h nüchtern war eine Frau. Seit 9 h nüchtern waren 3 Studienteilnehmer (1 Frau, 2 Männer). 10 h ohne Nahrungsaufnahme verbrachten 8 Probanden (3 Frauen, 5 Männer). Länger als 10 h nüchtern waren 12 Probanden (6 Frauen, 6 Männer) (Tabelle 1).

Zur Untersuchung des Einflusses der Nüchternperiode auf die LS wurden die Teilnehmer zusätzlich in drei Gruppen eingeteilt ( $3 \mathrm{~h}, 4-8 \mathrm{~h},>8 \mathrm{~h}$ Nüchternheit). Die Gruppe der Probanden mit einer Nüchternzeit von $3 \mathrm{~h}$ beinhaltete 32 Probanden (15 Frauen, 17 Männer). Die Gruppe mit 4-8 h umfasste 45 Teilnehmer (27 Männer, 18 Frauen) und die Gruppe mit $>8$ h 23 Probanden (10 Männer, 13 Frauen). Die folgende Tabelle zeigt die Verteilung des Probandenkollektivs bezüglich des Alters, BMIs, Alkoholkonsums, Herz- und Lebererkrankungen, Nüchternperiode und Medikamenteneinnahme, aufgeschlüsselt nach dem Gesamtkollektiv und nach dem Geschlecht (Tabelle 1). 
Tabelle 1: Charakteristika des Probandenkollektivs und Aufschlüsselung nach Geschlecht $(n=100)$.

\begin{tabular}{|c|c|c|c|}
\hline & Gesamt & Frauen & Männer \\
\hline Anzahl & 100 & 52 & 48 \\
\hline Alter (Jahre) & $25,8 \pm 6,1$ & $25,2 \pm 4,7$ & $26,4 \pm 7,2$ \\
\hline BMI $\left(\mathrm{kg} / \mathrm{m}^{2}\right)$ & $22,43 \pm 2,37$ & $21,43 \pm 1,91$ & $23,52 \pm 2,33$ \\
\hline Herzinsuffizienz & 0 & 0 & 0 \\
\hline Lebererkrankungen & 0 & 0 & 0 \\
\hline Sonographische Steatoseanzeichen & 1 & 1 & 0 \\
\hline $\begin{array}{l}\text { Alkoholkonsum/Tag: } \\
\text { Gruppe } 1(0 \mathrm{~g}) \\
\text { Gruppe } 2(0-10 \mathrm{~g}) \\
\text { Gruppe } 3(10-20 \mathrm{~g}) \\
\text { Gruppe } 4(20-30 \mathrm{~g}) \\
\text { Gruppe } 5(>30 \mathrm{~g})\end{array}$ & $\begin{array}{l}26 \\
50 \\
20 \\
4 \\
0 \\
\end{array}$ & $\begin{array}{l}15 \\
29 \\
8 \\
0 \\
0\end{array}$ & $\begin{array}{l}11 \\
21 \\
12 \\
4 \\
0 \\
\end{array}$ \\
\hline $\begin{array}{l}\text { Nüchternheit: } \\
3 \mathrm{~h} \\
4 \mathrm{~h} \\
5 \mathrm{~h} \\
6 \mathrm{~h} \\
7 \mathrm{~h} \\
8 \mathrm{~h} \\
9 \mathrm{~h} \\
10 \mathrm{~h} \\
>10 \mathrm{~h}\end{array}$ & $\begin{array}{l}32 \\
34 \\
5 \\
5 \\
0 \\
1 \\
3 \\
8 \\
12\end{array}$ & $\begin{array}{l}15 \\
21 \\
3 \\
2 \\
0 \\
1 \\
1 \\
3 \\
6\end{array}$ & $\begin{array}{l}17 \\
13 \\
2 \\
3 \\
0 \\
0 \\
2 \\
5 \\
6\end{array}$ \\
\hline $\begin{array}{l}\text { Nüchternheit: } \\
\text { Gruppe } 1 \text { (3 h) } \\
\text { Gruppe } 2 \text { (4 - } 8 \text { h) } \\
\text { Gruppe } 3 \text { (> } 8 \text { h) } \\
\end{array}$ & $\begin{array}{l}32 \\
45 \\
23\end{array}$ & $\begin{array}{l}15 \\
27 \\
10\end{array}$ & $\begin{array}{l}17 \\
18 \\
13 \\
\end{array}$ \\
\hline Medikamenteneinnahme & 30 & 26 & 4 \\
\hline
\end{tabular}

\subsection{Lebersteifigkeit (2D-SWE) bei lebergesunden Probanden}

Die folgenden Ausführungen beziehen sich auf die LSM der ersten Untersuchung vor Einnahme der Testmahlzeit (Nüchternmessung/ LS-Ausgangswert/ präprandiale Messung). Die Ergebnisse der ersten LSM waren normalverteilt. Im Durchschnitt aller Messwerte ergab sich eine LS von 4,80 $( \pm 0,94) \mathrm{kPa}$. Nach Höhe der durchschnittlichen LS-Ausgangwerte 
wurden zusätzlich drei Gruppen definiert. Eine Gruppe mit niedrigen LS-Ausgangswerten $(<4,5 \mathrm{kPa} ; \mathrm{n}=41)$, eine Gruppe mit normalen LS-Ausgangswerten $(4,5-5,5 \mathrm{kPa} ; \mathrm{n}=39)$ und eine Gruppe mit hochnormalen LS-Ausgangswerten (> 5,5 $\mathrm{kPa} ; \mathrm{n}=20)$.

\subsubsection{Einfluss des Geschlechts}

Die LSM der Frauen ergab eine durchschnittliche LS von 4,53 $( \pm 0,85) \mathrm{kPa}$, die der Männer eine mittlere LS von 5,09 $( \pm 0,95) \mathrm{kPa}$ (Tabelle 2). Es zeigte sich ein signifikanter geschlechtsspezifischer Unterschied in der LS $(\mathrm{p}=0,002)$ (Abbildung 5). Bei weiblichen Probanden wurde im Durchschnitt eine um 0,56 $( \pm 0,18) \mathrm{kPa}$ niedrigere LS gemessen als bei männlichen Probanden.

Tabelle 2: Mittelwerte der LS-Ausgangswerte ( \pm SD) und Minimum bis Maximum der LS in kPa für das Gesamtkollektiv und geschlechtsspezifisch $(n=100$, Frauen $n=52$, Männer $n=48)$.

\begin{tabular}{l|l|l} 
& LS-Ausgangswert $(\mathbf{k P a})$ & LS Minimum - Maximum $(\mathbf{k P a})$ \\
\hline Gesamt & $4,80( \pm 0,94)$ & $2,66-7,02$ \\
\hline Frauen & $4,53( \pm 0,85)$ & $2,77-6,85$ \\
\hline Männer & $5,09( \pm 0,95)$ & $2,66-7,02$
\end{tabular}

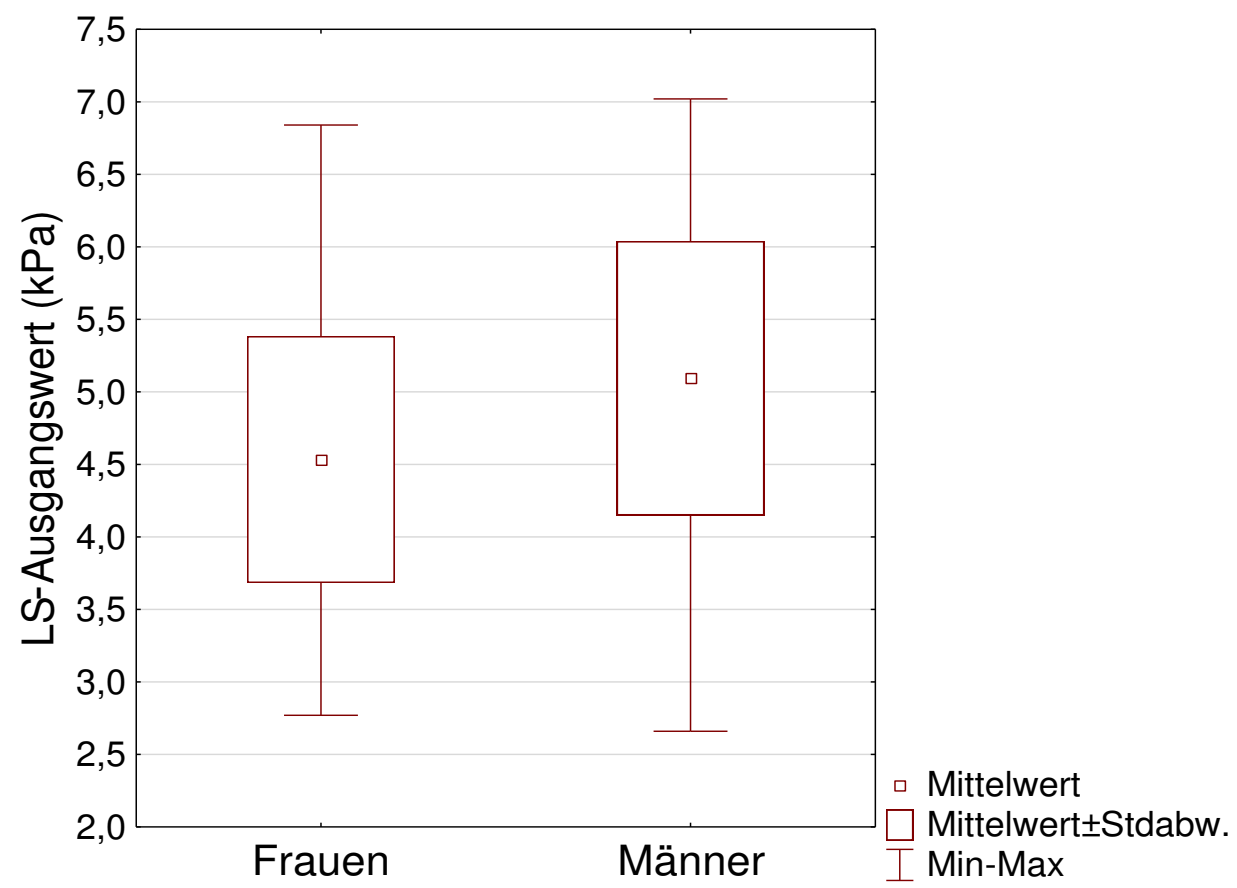

Abbildung 5: Boxplot-Diagramme der Mittelwerte der LS-Ausgangswerte weiblicher $(n=52)$ und männlicher Probanden $(\mathrm{n}=48)$ in $\mathrm{kPa}(\mathrm{p}=0,002)$. 


\subsubsection{Einfluss des Alters, des BMIs und des Alkoholkonsums}

Bezüglich des Alters der Probanden lag keine Normalverteilung vor. Unter Anwendung des Spearman-Korrelationskoeffizienten zeigte sich keine signifikante Korrelation zwischen der durchschnittlichen LS und dem Alter der Studienteilnehmer (Spearman r = - 0,094, p = 0,351). Der BMI, welcher eine Normalverteilung zeigte, hatte ebenfalls keinen signifikanten Einfluss auf die LS ( $r=0,119, \mathrm{p}=0,237)$. Bei Probanden, die ein Medikament eingenommen haben, wurde eine mittlere LS von 4,79 $( \pm 0,71) \mathrm{kPa}$ beobachtet, bei Probanden ohne Medikamenteneinnahme im Durchschnitt eine LS von 4,81 $( \pm 1,02) \mathrm{kPa}$. Es zeigte sich kein signifikanter Unterschied im Vergleich beider Gruppen $(\mathrm{p}=0,906)$. Der Alkoholkonsum wurde in fünf Gruppen unterteilt $(0 \mathrm{~g} / \mathrm{d}, 0-10 \mathrm{~g} / \mathrm{d}, 10-20 \mathrm{~g} / \mathrm{d}, 20-30 \mathrm{~g} / \mathrm{d},>30 \mathrm{~g} / \mathrm{d})$. In Gruppe 1 wurde im Durchschnitt eine LS von 4,79 $( \pm 0,76) \mathrm{kPa}$, in Gruppe 2 eine LS von 4,67 $( \pm 0,93)$ $\mathrm{kPa}$, in Gruppe 3 eine LS von 4,96 $( \pm 1,12) \mathrm{kPa}$ und in Gruppe 4 eine LS von 5,78 $( \pm 0,41)$ $\mathrm{kPa}$ gemessen. Anhand des angegebenen Alkoholkonsums wurde keiner der Probanden der Gruppe 5 zugeordnet (Tabelle 6). Es zeigte sich kein signifikanter Unterschied im Vergleich aller Gruppen $(\mathrm{p}=0,109)$.

\subsubsection{Einfluss der Nüchternperiode}

Bezüglich des Einflusses der Nüchternperiode zeigte sich mithilfe einer Regressionsanalyse eine Änderung der LS von $-0,05 \mathrm{kPa}$, bei einer Verlängerung der Nüchternzeit um eine Stunde. Dieser Effekt zeigte keine Signifikanz ( $b=-0,05, p=0,072)$. Zur weiteren Untersuchung des Einflusses der Nüchternperiode wurden die Teilnehmer in drei Gruppen unterteilt (s. 3.1). Die durchschnittlich höchste LS wurde in Gruppe 1 (3 h) gemessen. Sie lag hier im Mittel bei 4,92 ( $\pm 0,95) \mathrm{kPa}$. In Gruppe $2(4-8 \mathrm{~h})$ wurde im Durchschnitt eine LS von 4,90 $( \pm 0,94) \mathrm{kPa}$ beobachtet. Die niedrigsten LS wurden in Gruppe $3(>8 \mathrm{~h})$ gemessen. Die durchschnittliche LS betrug hier 4,44 $( \pm 0,84) \mathrm{kPa}$ (Abbildung 6, Tabelle 7). Die ANOVA zeigte im Vergleich der LS aller drei Nüchterngruppen keine Signifikanz $(p=0,103)$. Die $p$ Werte zwischen den einzelnen Gruppen werden in Tabelle 3 gezeigt. 


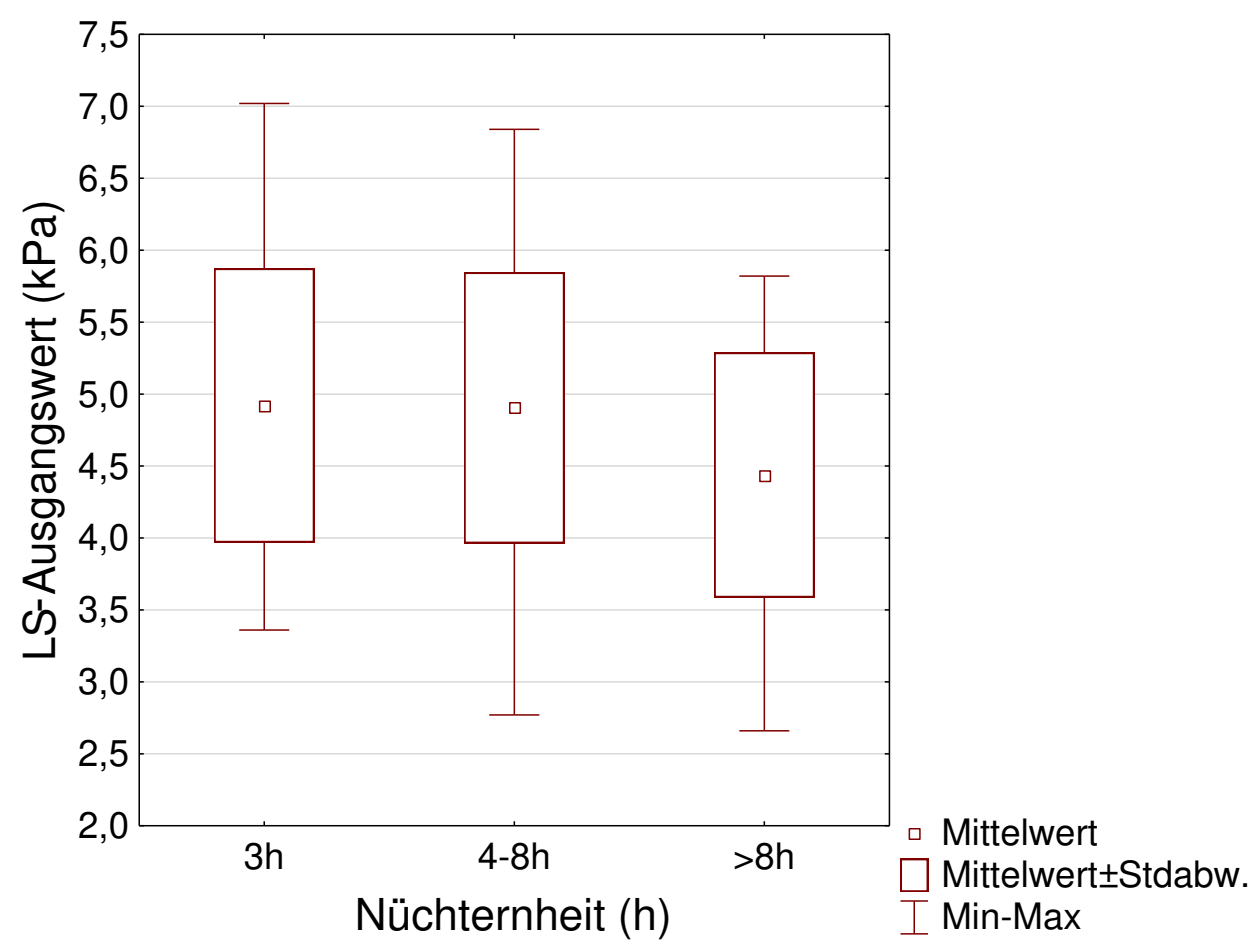

Abbildung 6: Boxplot-Diagramme des LS-Ausgangswerts in kPa gruppiert nach Nüchternheit in $\mathrm{h}$ (Gruppe 1 (3 h), $\mathrm{n}=32$; Gruppe $2(4-8 \mathrm{~h}), \mathrm{n}=45$; Gruppe $3(>8 \mathrm{~h}), \mathrm{n}=23$ ).

Tabelle 3: p-Werte der durchschnittlichen LS für verschiedene Nüchterngruppen (Gruppe 1 (3 h), $\mathrm{n}$ $=32$; Gruppe $2(4-8 \mathrm{~h}), \mathrm{n}=45$; Gruppe $3(>8 \mathrm{~h}), \mathrm{n}=23)$.

\begin{tabular}{l|l|l|l} 
Nüchternheit & Gruppe 1 $(3 \mathrm{~h})$ & Gruppe 2 $(4-8 \mathrm{~h})$ & Gruppe 3 $(>8 \mathrm{~h})$ \\
\hline Gruppe 1 $(3 \mathrm{~h})$ & & 0,997 & 0,141 \\
\hline Gruppe 2 $(4-8 \mathrm{~h})$ & 0,997 & & 0,125 \\
\hline Gruppe 3 $>$ 8 h) & 0,141 & 0,125 &
\end{tabular}

\subsubsection{IQR-Wert und der Einfluss des Geschlechts, der Nüchternperiode und des LS- Ausgangswerts}

Der IQR-Wert wurde als Qualitätskriterium der Messungen verwendet. Vor Nahrungsaufnahme wurde ein durchschnittlicher IQR-Wert von $0,51( \pm 0,21) \mathrm{kPa}$ gemessen. Bei weiblichen Probanden lag dieser im Mittel bei 0,46 $( \pm 0,21) \mathrm{kPa}$, bei Männern bei 0,57 $( \pm 0,20)$ $\mathrm{kPa}$. Der geschlechtsspezifische Vergleich ergab einen signifikanten Unterschied $(\mathrm{p}=$ 0,006). Der IQR-Wert der Messungen bei männlichen Probanden war im Durchschnitt um 0,11 kPa höher als bei weiblichen Probanden. Der Vergleich der drei Nüchterngruppen, bezogen auf den IQR-Wert vor Nahrungsaufnahme zeigte keinen signifikanten Unterschied ( $p$ $=0,570)$. Bei Probanden in Gruppe $1(3 \mathrm{~h})$ wurde ein durchschnittlicher IQR-Wert von 0,50 
$( \pm 0,20) \mathrm{kPa}$ gemessen, in Gruppe $2(4-8 \mathrm{~h})$ von $0,54( \pm 0,23) \mathrm{kPa}$ und in Gruppe $3(>8 \mathrm{~h})$ von $0,48( \pm 0,19) \mathrm{kPa}$. Eine signifikante Korrelation der durchschnittlichen Ausgangswerte der LS und der IQR-Werte konnte nicht beobachtet werden $(r=0,121, p=0,232)$.

\subsection{Abhängigkeit der Lebersteifigkeit (2D-SWE) von der Nahrungsaufnahme}

Zunächst wurden die Mittelwerte der LS vor und nach Nahrungsaufnahme miteinander verglichen, ohne Berücksichtigung weiterer Einflussfaktoren. Bei vier Probanden wurde eine Abnahme der LS nach Nahrungsaufnahme festgestellt. Zwei Teilnehmer zeigten keine Veränderung der LS. Alle anderen 94 Studienteilnehmer zeigten einen Anstieg der LS. Vor Nahrungsaufnahme betrug die LS im Mittel 4,80 $( \pm 0,94) \mathrm{kPa}$. Nach Nahrungsaufnahme wurde eine durchschnittliche LS von 5,74 $( \pm 0,94) \mathrm{kPa}$ gemessen. Es ergab sich ein hochsignifikanter Unterschied ( $<$ 0,001) (Tabelle 4, Abbildung 7).

Tabelle 4: durchschnittliche LS ( \pm SD) und Minimum bis Maximum in kPa prä- und postprandial und Berechnung des $\mathrm{p}$-Werts $(\mathrm{n}=100)$.

\begin{tabular}{l|l|l|l} 
& präprandial & postprandial & p-Wert \\
\hline LS $(\mathrm{kPa})$ & $4,80( \pm 0,94)$ & $5,74( \pm 0,94)$ & $<0,001$ \\
\hline $\begin{array}{l}\text { LS Minimum - } \\
\text { Maximum }(\mathrm{kPa})\end{array}$ & $2,66-7,02$ & $3,12-8,14$ &
\end{tabular}

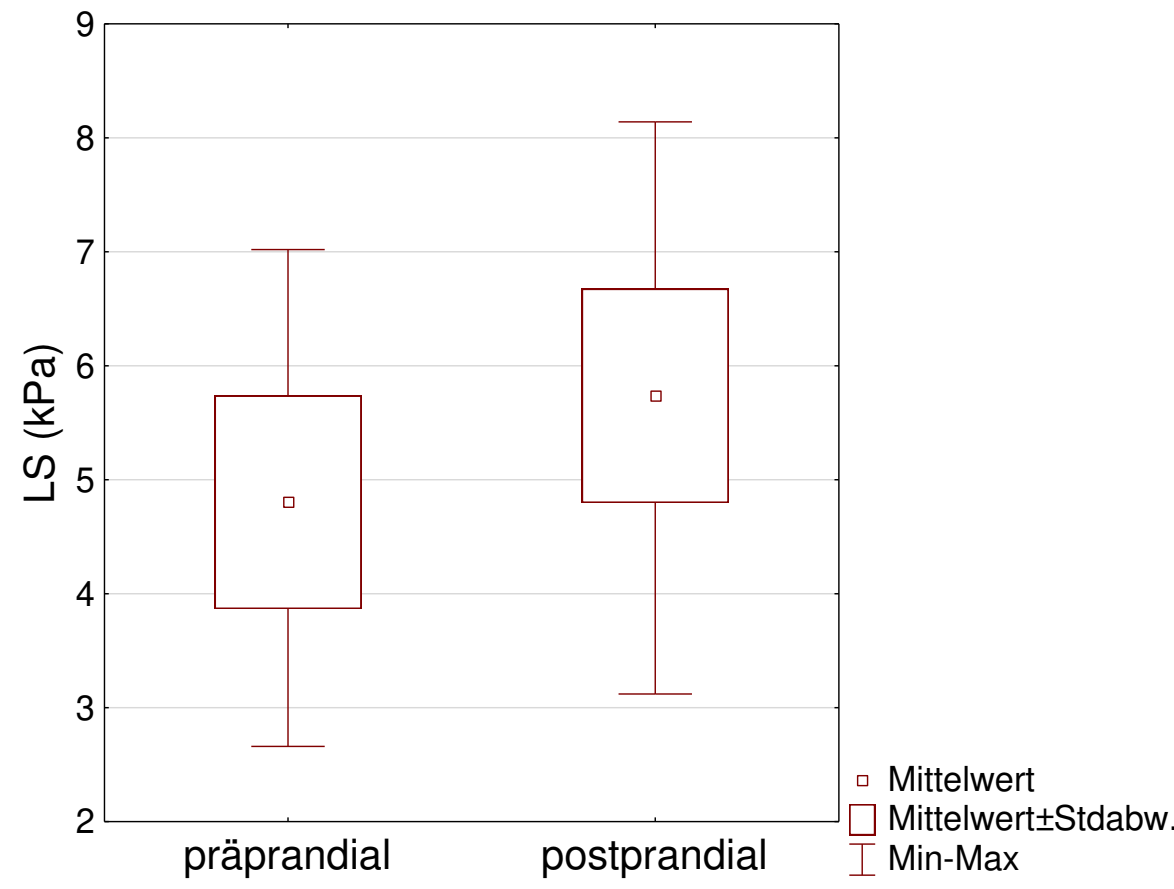

Abbildung 7: Boxplot-Diagramme der prä- und postprandialen Mittelwerte der LS in $\mathrm{kPa}(\mathrm{p}<0,001$, $\mathrm{n}=100)$. 
Bei weiblichen Probanden wurde präprandial durchschnittlich eine LS von 4,53 $( \pm 0,85) \mathrm{kPa}$ und postprandial von 5,41 $( \pm 0,95) \mathrm{kPa}$ gemessen $(\mathrm{p}<0,001)$. Bei männlichen Probanden lag die mittlere LS vor Nahrungsaufnahme bei 5,09 $( \pm 0,95) \mathrm{kPa}$ und nach Nahrungsaufnahme bei 6,09 $( \pm 0,79) \mathrm{kPa}(\mathrm{p}<0,001)$.

Zur Untersuchung potenzieller Einflussgrößen auf den nahrungsbedingten Anstieg der LS wurde für jeden Probanden die Differenz zwischen den beiden LSM (prä- und postprandial) errechnet. Diese Differenz wird im Folgenden als LSA bezeichnet. Im Durchschnitt ergab sich ein Anstieg der LS um 0,93 $( \pm 0,74) \mathrm{kPa}(21,6 \%)$ nach Nahrungsaufnahme.

\subsubsection{Einfluss des Geschlechts auf den postprandialen LSA}

Es zeigte sich kein signifikanter Einfluss des Geschlechts auf den nahrungsbedingten LSA $(\mathrm{p}=0,404)$. Bei weiblichen Probanden wurde durchschnittlich ein LSA von 0,87 $( \pm 0,68)$ $\mathrm{kPa}(20,6 \%)$ beobachtet. Bei Männern betrug dieser im Mittel 1,00 $( \pm 0,80) \mathrm{kPa}(22,7 \%)$ (Tabelle 5).

Tabelle 5: durchschnittlicher postprandialer LSA $( \pm$ SD) und Minimum bis Maximum des postprandialen LSA in kPa für das Gesamtkollektiv und geschlechtsspezifisch ( $n=100$, Frauen n = 52, Männer $\mathrm{n}=48$ ).

\begin{tabular}{l|l|l} 
& postprandialer LSA $(\mathrm{kPa})$ & LSA Minimum - Maximum $(\mathrm{kPa})$ \\
\hline Gesamt & $0,93( \pm 0,74)$ & $-0,57-3,16$ \\
\hline Frauen & $0,87( \pm 0,68)$ & $-0,57-2,34$ \\
\hline Männer & $1,00( \pm 0,80)$ & $-0,46-3,16$
\end{tabular}

\subsubsection{Einfluss des Alters und des BMIs auf den postprandialen LSA}

Das Alter der Studienteilnehmer korrelierte nicht signifikant mit dem nahrungsbedingten LSA (Spearman $r=0,110, p=0,274$ ). Zur Untersuchung des Einflusses des BMIs wurde eine Regressionsanalyse durchgeführt. Dabei wurde beobachtet, dass eine Erhöhung des BMIs um $1 \mathrm{~kg} / \mathrm{m}^{2}$, einen durchschnittlichen LSA von 0,08 kPa zur Folge hat. Der BMI zeigte demnach einen signifikanten Einfluss auf den nahrungsbedingten LSA $(b=0,08, p=0,010)$ (Abbildung 8). 


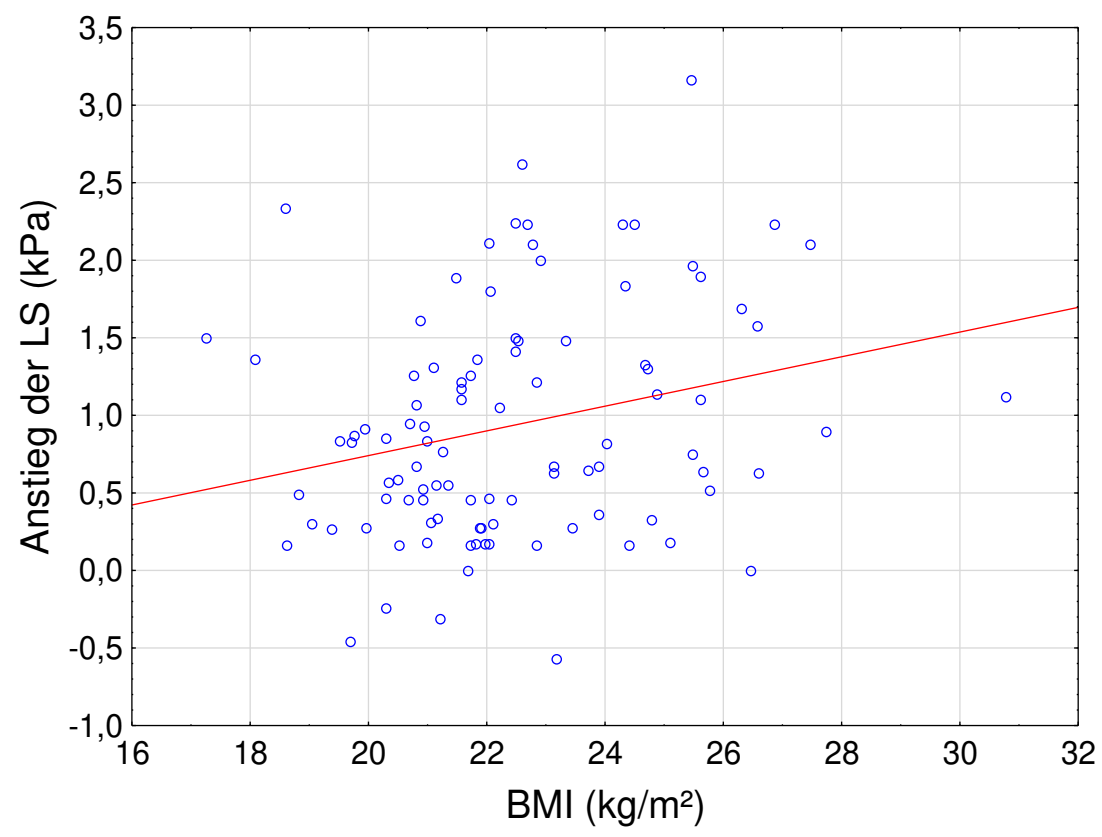

Abbildung 8: Scatterplot-Diagramm: BMI in $\mathrm{kg} / \mathrm{m}^{2}$ versus postprandialer LSA in $\mathrm{kPa}(\mathrm{b}=0,08, \mathrm{p}$ $=0,010, \mathrm{n}=100)$.

\subsubsection{Einfluss der Medikamenteneinnahme und des Alkoholkonsums auf den postprandialen LSA}

Der Vergleich des LSA von Probanden mit und ohne Medikamenteneinnahme zeigte keine Signifikanz $(\mathrm{p}=0,770)$. Der durchschnittliche LSA in der Gruppe ohne Medikamenteneinnahme lag bei $0,95( \pm 0,80) \mathrm{kPa}$, der der Gruppe mit Medikamenteneinnahme bei 0,90 $( \pm$ $0,59) \mathrm{kPa}$. Der Vergleich der vier Alkoholkonsumgruppen blieb ohne Signifikanz $(\mathrm{p}=$ 0,699). In Gruppe 1 wurde ein durchschnittlicher LSA von 0,85 $( \pm 0,85) \mathrm{kPa}$, in Gruppe 2 von $1,01( \pm 0,73) \mathrm{kPa}$, in Gruppe 3 von $0,89( \pm 0,69) \mathrm{kPa}$ und in Gruppe 4 von 0,67 $( \pm 0,49)$ kPa gemessen (Tabelle 6).

Tabelle 6: durchschnittliche LS $( \pm$ SD) prä- und postprandial und der durchschnittliche postprandiale LSA $( \pm \mathrm{SD}$ ) in $\mathrm{kPa}$ aufgeschlüsselt nach Alkoholkonsumgruppen (Gruppe $1 \mathrm{n}=26$, Gruppe $2 \mathrm{n}=$ 50, Gruppe $3 \mathrm{n}=20$, Gruppe $4 \mathrm{n}=4$ ).

\begin{tabular}{l|l|l|l} 
Alkoholkonsum & $\begin{array}{l}\text { LS präprandial } \\
(\mathrm{kPa})\end{array}$ & $\begin{array}{l}\text { LS postprandial } \\
(\mathrm{kPa})\end{array}$ & LSA $(\mathrm{kPa})$ \\
\hline Gruppe $1(0 \mathrm{~g} / \mathrm{d})$ & $4,79( \pm 0,76)$ & $5,65( \pm 0,89)$ & $0,85( \pm 0,85)$ \\
\hline Gruppe $2(0-10 \mathrm{~g} / \mathrm{d})$ & $4,67( \pm 0,93)$ & $5,68( \pm 0,91)$ & $1,01( \pm 0,73)$ \\
\hline Gruppe $3(10-20 \mathrm{~g} / \mathrm{d})$ & $4,96( \pm 1,12)$ & $5,86( \pm 1,03)$ & $0,89( \pm 0,69)$ \\
\hline Gruppe $4(20-30 \mathrm{~g} / \mathrm{d})$ & $5,78( \pm 0,41)$ & $6,45( \pm 0,38)$ & $0,67( \pm 0,49)$
\end{tabular}




\subsubsection{Einfluss der Nüchternperiode auf den postprandialen LSA}

Eine Regressionsanalyse zeigte, dass aus einer Verlängerung der Nüchternperiode um eine Stunde ein durchschnittlicher LSA von 0,01 kPa resultiert. Diese Beobachtung zeigte keine Signifikanz $(b=0,01, p=0,667)$. Auch der Vergleich der drei Nüchterngruppen blieb ohne Signifikanz $(p=0,784)$. Die im Mittel größten nahrungsbedingten LSA wurden in Gruppe 3 (> $8 \mathrm{~h}$ ) beobachtet. Hier betrug der durchschnittliche LSA 1,00 ( $\pm 0,94) \mathrm{kPa}$. In Gruppe 2 (4 - 8 h) 0,95 $( \pm 0,64) \mathrm{kPa}$ und in Gruppe $1(3 \mathrm{~h}) 0,86( \pm 0,74) \mathrm{kPa}$ (Tabelle 7, Abbildung 9). $\mathrm{Zu}$ den $\mathrm{p}$-Werten der verglichenen Nüchterngruppen siehe Tabelle 8.

Tabelle 7: durchschnittliche LS ( \pm SD) prä- und postprandial und der durchschnittliche postprandiale LSA $( \pm \mathrm{SD}$ ) in $\mathrm{kPa}$ aufgeschlüsselt nach Nüchterngruppen (Gruppe $1 \mathrm{n}=32$, Gruppe $2 \mathrm{n}=45$, Gruppe $3 \mathrm{n}=23)$.

\begin{tabular}{l|l|l|l} 
Nüchternheit & $\begin{array}{l}\text { LS präprandial } \\
(\mathrm{kPa})\end{array}$ & $\begin{array}{l}\text { LS postprandial } \\
(\mathrm{kPa})\end{array}$ & LSA $(\mathrm{kPa})$ \\
\hline Gruppe $1(3 \mathrm{~h})$ & $4,92( \pm 0,95)$ & $5,79( \pm 1,06)$ & $0,86( \pm 0,74)$ \\
\hline Gruppe $2(4-8 \mathrm{~h})$ & $4,90( \pm 0,94)$ & $5,85( \pm 0,86)$ & $0,95( \pm 0,64)$ \\
\hline Gruppe $3(>8 \mathrm{~h})$ & $4,44( \pm 0,85)$ & $5,44( \pm 0,89)$ & $1,00( \pm 0,94)$
\end{tabular}

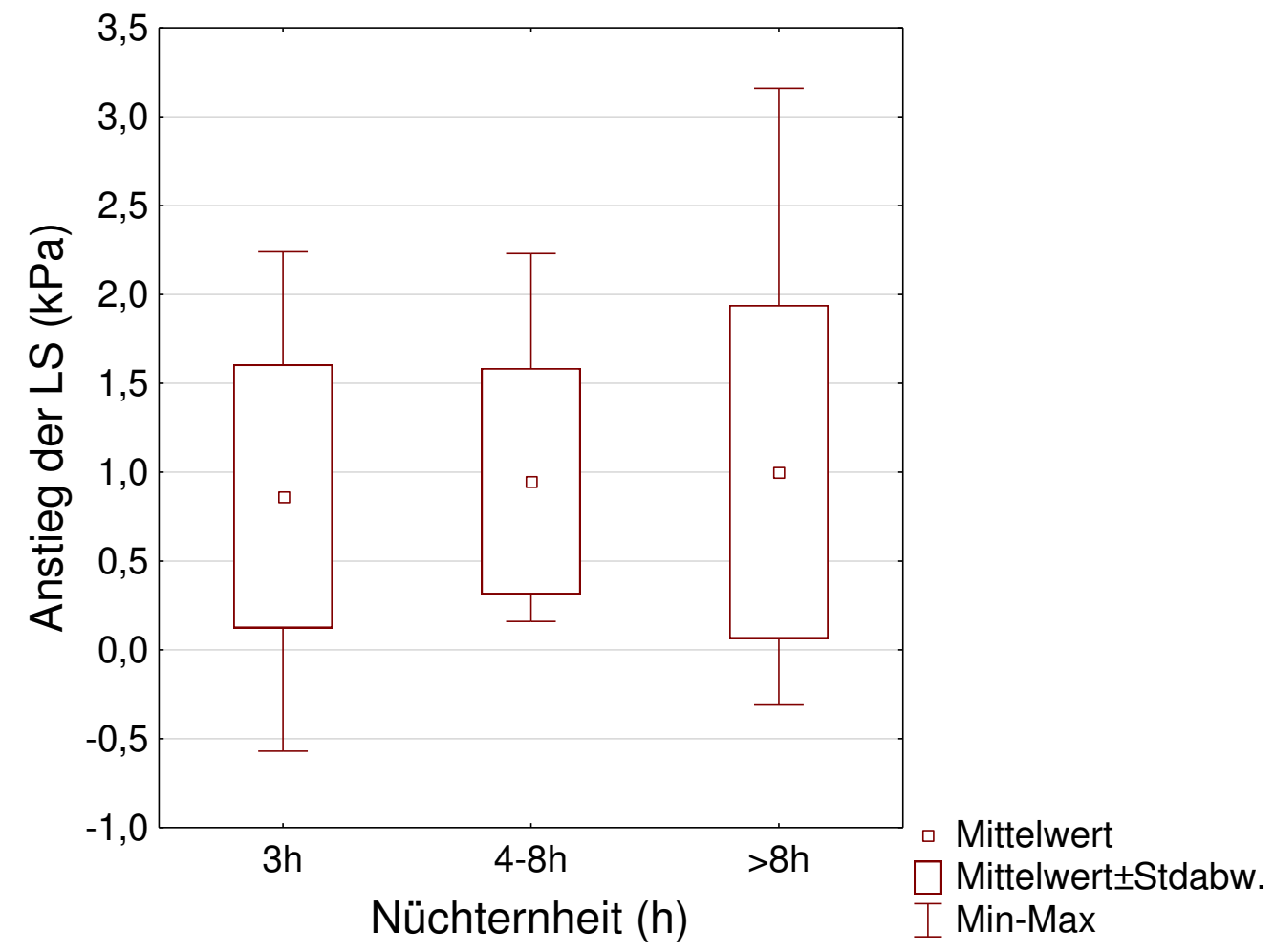

Abbildung 9: Boxplot-Diagramme des postprandialen LSA in kPa gruppiert nach Nüchternheit in $\mathrm{h}$ (Gruppe 1 (3 h), n = 32; Gruppe $2(4-8 \mathrm{~h}), \mathrm{n}=45$; Gruppe 3 (> $8 \mathrm{~h}), \mathrm{n}=23$ ). 
Tabelle 8: $p$-Werte des durchschnittlichen postprandialen LSA für verschiedene Nüchterngruppen (Gruppe 1 (3 h), $\mathrm{n}=32$; Gruppe $2(4-8 \mathrm{~h}), \mathrm{n}=45$; Gruppe $3(>8 \mathrm{~h}), \mathrm{n}=23$ ).

\begin{tabular}{l|l|l|l} 
Nüchternheit & Gruppe 1 $(3 \mathrm{~h})$ & Gruppe 2 $(4-8 \mathrm{~h})$ & Gruppe 3 $(>8 \mathrm{~h})$ \\
\hline Gruppe 1 $(3 \mathrm{~h})$ & & 0,876 & 0,779 \\
\hline Gruppe 2 $(4-8 \mathrm{~h})$ & 0,876 & & 0,959 \\
\hline Gruppe 3 $>$ 8 h) & 0,779 & 0,959 &
\end{tabular}

\subsubsection{Einfluss des LS-Ausgangswerts auf den postprandialen LSA}

Zusätzlich wurde der Einfluss des LS-Ausgangswerts auf den nahrungsbedingten LSA untersucht. Dieser Zusammenhang erwies sich als hochsignifikant. Die durchschnittlichen Messwerte der LS-Ausgangswerte und der absoluten LSA nach Nahrungsaufnahme korrelierten signifikant negativ $(r=-0,392, p<0,001)$. Nach Cohen handelte es sich hierbei um eine mittlere Effektstärke. Mithilfe einer weiterführenden Regressionsanalyse wurde deutlich, dass bei einer Erhöhung des LS-Ausgangswerts um $1 \mathrm{kPa}$, der absolute LSA nach Nahrungsaufnahme um $-0,31 \mathrm{kPa}$ sinkt $(\mathrm{b}=-0,31, \mathrm{p}<0,001)$ (Abbildung 10).

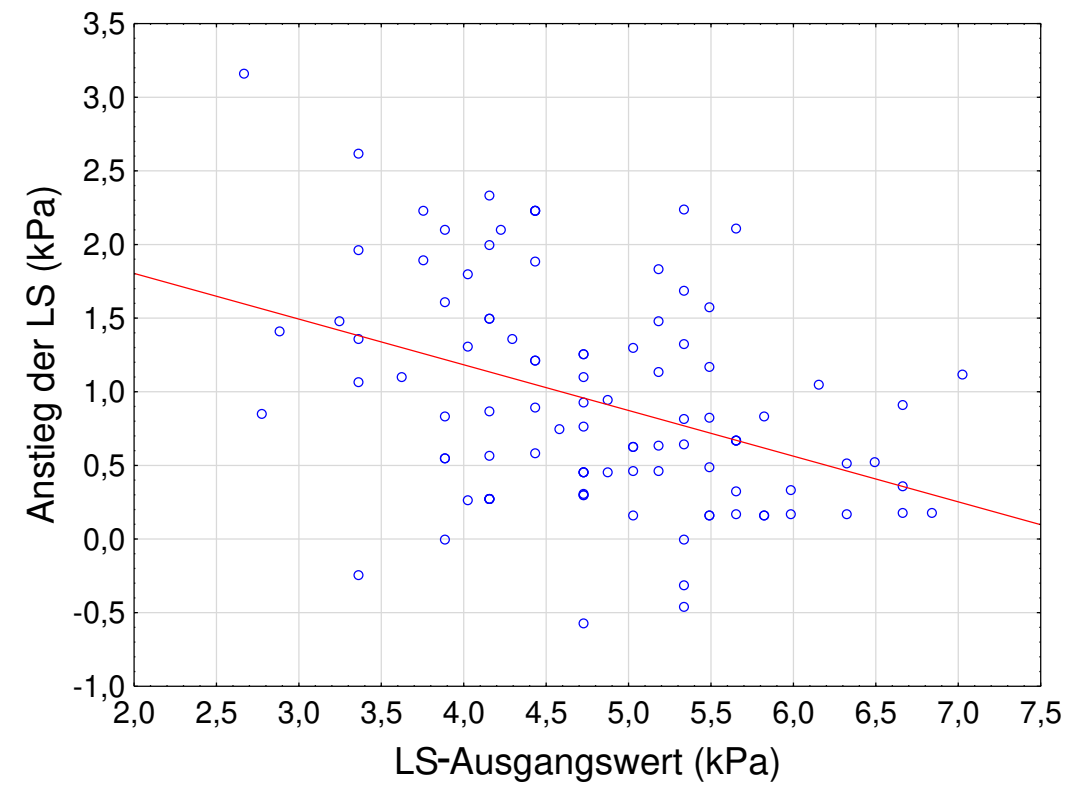

Abbildung 10: Scatterplot-Diagramm: LS-Ausgangswert versus postprandialer LSA in $\mathrm{kPa}(\mathrm{b}=-$ $0,31, \mathrm{p}<0,001, \mathrm{n}=100)$.

Bezogen auf die drei nach Höhe der LS-Ausgangswerte definierten Gruppen, zeigten sich signifikante Unterschiede $(\mathrm{p}<0,001)$. Probanden mit niedrigen LS-Ausgangswerten $(<4,5$ $\mathrm{kPa})$ zeigten einen durchschnittlichen LSA von $1,31( \pm 0,80) \mathrm{kPa}(34,6 \%)$, Teilnehmer mit normalen LS-Ausgangswerten $(4,5-5,5 \mathrm{kPa})$ von $0,73( \pm 0,61) \mathrm{kPa}(14,3 \%)$ und Probanden 
mit hochnormalen LS-Ausgangswerten $(>5,5 \mathrm{kPa})$ von $0,57( \pm 0,48) \mathrm{kPa}(9,3 \%)$ (Abbildung 11). Probanden mit niedrigen LS-Ausgangswerten zeigten, bezüglich der durchschnittlichen LSA, signifikante Unterschiede im Vergleich zu Probanden mit normale bzw. hochnormalen LS-Ausgangswerten. Der Vergleich der Teilnehmer mit normalen und hochnormalen LS-Ausgangswerten blieb ohne Signifikanz. Die p-Werte der einzelnen LS-Ausgangswerte sind in Tabelle 9 dargestellt.

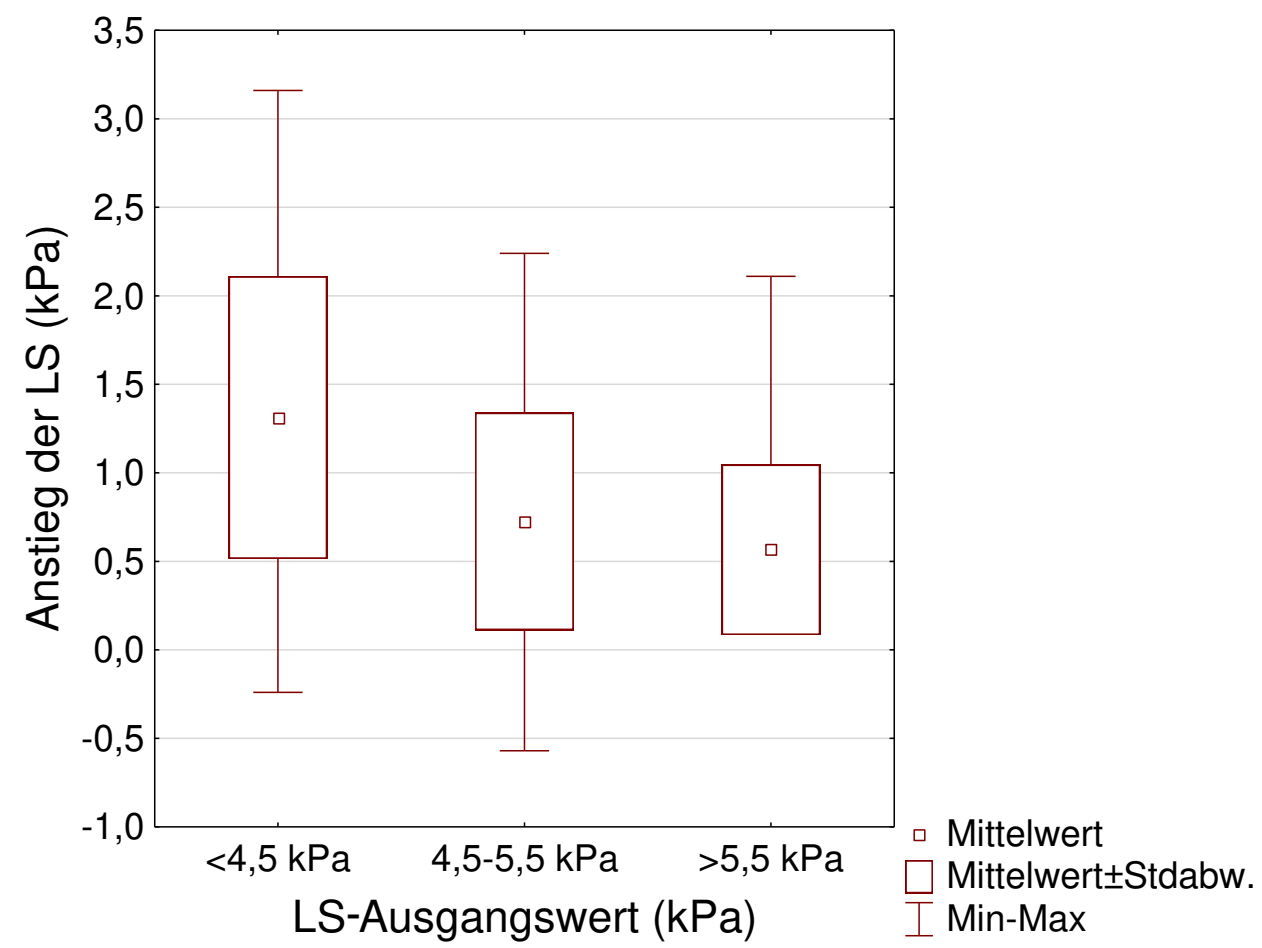

Abbildung 11: Boxplot-Diagramme des postprandialen LSA in kPa gruppiert nach LS-Ausgangswerten in $\mathrm{kPa}$ (niedrig $(<4,5 \mathrm{kPa}), \mathrm{n}=41$; normal $(4,5-5,5 \mathrm{kPa}), \mathrm{n}=39$; hochnormal $(>5,5 \mathrm{kPa})$, $\mathrm{n}=20)$.

Tabelle 9: p-Werte des durchschnittlichen postprandialen LSA für verschiedene LS-Ausgangswerte (niedrig $(<4,5 \mathrm{kPa}), \mathrm{n}=41$; normal $(4,5-5,5 \mathrm{kPa}), \mathrm{n}=39$; hochnormal $(>5,5 \mathrm{kPa}), \mathrm{n}=20)$.

\begin{tabular}{l|l|l|l} 
LS-Ausgangswert & $\begin{array}{l}\text { Niedrig } \\
(<4,5 \mathrm{kPa})\end{array}$ & $\begin{array}{l}\text { Normal } \\
(4,5-5,5 \mathrm{kPa})\end{array}$ & $\begin{array}{l}\text { Hochnormal } \\
(>5,5 \mathrm{kPa})\end{array}$ \\
\hline Niedrig $(<4,5 \mathrm{kPa})$ & & $<0,001$ & $<0,001$ \\
\hline Normal $(4,5-5,5 \mathrm{kPa})$ & $<0,001$ & & 0,664 \\
\hline Hochnormal $(>5,5 \mathrm{kPa})$ & $<0,001$ & 0,664 &
\end{tabular}

\subsubsection{Fibrosestadien des Probandenkollektivs gemäß definierten Cut-off-Werten}

Gemäß den Cut-off-Werten für die Leberfibrosediagnostik mit LOGIQ E9 (Bende et al. 2017: $F \geq 2,6,7 \mathrm{kPa} ; \mathrm{F} \geq 3,8,2 \mathrm{kPa} ; \mathrm{F}=4,9,3 \mathrm{kPa}$ ) erreichten 98 Probanden Fibrosegrad $\mathrm{F}$ 
0 /F 1 und 2 Probanden Fibrosegrad F $\geq 2$ vor Einnahme der Testmahlzeit. Nach Nahrungsaufnahme zeigten 87 Teilnehmer Fibrosegrad F 0/F 1 und 13 Teilnehmer Fibrosegrad F $\geq 2$. Keiner der Probanden erreichte vor oder nach Nahrungsaufnahme, Fibrosegrad F $\geq 3$ oder F 4. Somit wurde bei $11 \%$ aller Probanden, unter Berücksichtigung der Cut-off-Werte, nach Nahrungsaufnahme ein höherer Fibrosegrad beobachtet.

\subsection{Abhängigkeit des IQR-Werts von der Nahrungsaufnahme}

Der IQR-Wert wurde als Qualitätskriterium der Messungen verwendet. Um den Einfluss der Nahrungsaufnahme auf den IQR-Wert zu untersuchen, wurden zunächst die IQR-Werte jedes Probanden vor und nach Nahrungsaufnahme miteinander verglichen. Bei 46 Probanden sank der IQR-Wert postprandial, bei einem Teilnehmer gab es keine Veränderung und bei 53 Probanden stieg dieser nach Nahrungsaufnahme. Durchschnittlich ergab sich vor Nahrungsaufnahme ein IQR-Wert von $0,51( \pm 0,21) \mathrm{kPa}$ und nach Nahrungsaufnahme von 0,54 $( \pm 0,22) \mathrm{kPa}$. Es wurde kein signifikanter Unterschied vor und nach Nahrungsaufnahme festgestellt $(\mathrm{p}=0,378)$. Um die Veränderungen des IQR-Werts nach Nahrungsaufnahme genauer zu untersuchen, wurde für jeden Probanden die Differenz zwischen den IQR-Werten beider Messungen (prä- und postprandial) errechnet. Im Durchschnitt zeigte sich ein Anstieg um 0,03 ( $\pm 0,32) \mathrm{kPa}$ nach Nahrungsaufnahme (Tabelle 10).

Tabelle 10: durchschnittlicher IQR-Wert $( \pm$ SD) und Minimum bis Maximum in kPa prä- und postprandial und die durchschnittliche prä- und postprandiale Differenz $(\mathrm{n}=100)$.

\begin{tabular}{l|l|l|l} 
& präprandial & postprandial & IQR Änderung \\
\hline IQR $(\mathrm{kPa})$ & $0,51( \pm 0,21)$ & $0,54( \pm 0,22)$ & $0,03( \pm 0,32)$ \\
\hline $\begin{array}{l}\text { IQR Minimum - Ma- } \\
\text { ximum }(\mathrm{kPa})\end{array}$ & $0,07-1,06$ & $0,07-1,29$ & $-0,64-0,98$
\end{tabular}

\subsection{Abhängigkeit des Pfortaderblutflusses von der Nahrungsaufnahme}

Die Geschwindigkeit des PBF vor Nahrungsaufnahme betrug im Durchschnitt 22,34 $( \pm 3,43)$ $\mathrm{cm} / \mathrm{s}$. Bei Frauen wurde im Mittel ein PBF von 22,81 $( \pm 3,79) \mathrm{cm} / \mathrm{s}$ gemessen, bei Männern von $21,83( \pm 2,94) \mathrm{cm} / \mathrm{s}$. Der geschlechtsspezifische Vergleich blieb ohne Signifikanz $(\mathrm{p}=$ 0,151). Auch zwischen den Nüchterngruppen zeigte sich kein signifikanter Unterschied ( $\mathrm{p}$ $=0,187)$. In Gruppe 1 (3 h) ergab sich im Durchschnitt ein PBF von 21,42 $( \pm 3,26) \mathrm{cm} / \mathrm{s}$, in Gruppe $2(4-8 \mathrm{~h})$ von $22,75( \pm 3,70) \mathrm{cm} / \mathrm{s}$ und in Gruppe $3(>8 \mathrm{~h})$ von $22,81( \pm 2,95) \mathrm{cm} / \mathrm{s}$. Bezüglich der Analyse des Einflusses der Nahrungsaufnahme auf den PBF wurden die PBF Messungen jedes Patienten vor und nach Nahrungsaufnahme miteinander verglichen. Bei allen Probanden wurde ein Anstieg des PBF beobachtet. Präprandial wurde im Durchschnitt 
ein PBF von 22,34 $( \pm 3,43) \mathrm{cm} / \mathrm{s}$ gemessen, postprandial im Mittel 35,98 $( \pm 5,32) \mathrm{cm} / \mathrm{s}(\mathrm{Ab}-$ bildung 12). Der Vergleich ergab einen signifikanten Unterschied ( $\mathrm{p}<0,001)$. Um die Abhängigkeit der nahrungsbedingten Erhöhung des PBF zu untersuchen, wurde für jeden Probanden die Differenz zwischen den beiden PBF Messungen (prä- und postprandial) errechnet. Im Durchschnitt ergab sich ein Anstieg des PBF nach Nahrungsaufnahme von 13,65 ( \pm $5,05) \mathrm{cm} / \mathrm{s}(63,5 \%)$ (Tabelle 11).

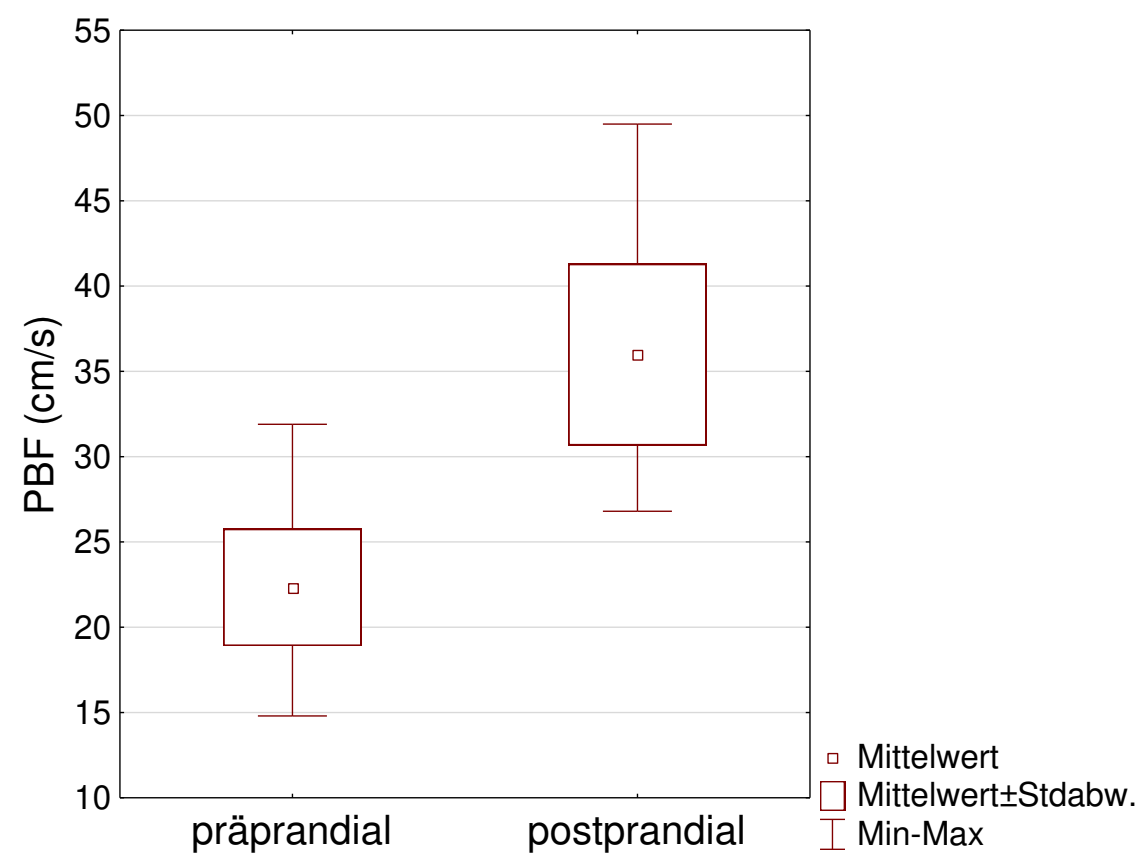

Abbildung 12: Boxplot-Diagramme der prä- und postprandialen Mittelwerte des PBF in $\mathrm{cm} / \mathrm{s}(\mathrm{p}<$ $0,001, \mathrm{n}=100)$.

Tabelle 11: durchschnittlicher PBF ( \pm SD) und Minimum bis Maximum prä- und postprandial und der durchschnittliche postprandiale Anstieg des PBF in cm/s $(\mathrm{n}=100)$.

\begin{tabular}{l|l|l|l} 
& präprandial & postprandial & PBF Anstieg \\
\hline PBF $(\mathrm{cm} / \mathrm{s})$ & $22,34( \pm 3,43)$ & $35,98( \pm 5,32)$ & $13,65( \pm 5,05)$ \\
\hline $\begin{array}{l}\text { PBF Minimum - Maxi- } \\
\text { mum }(\mathrm{cm} / \mathrm{s})\end{array}$ & $14,80-31,90$ & $26,80-49,50$ & $2,50-26,00$
\end{tabular}

Bei weiblichen Probanden lag der Anstieg des PBF im Mittel bei 13,36 ( $\pm 5,31) \mathrm{cm} / \mathrm{s}(61,4$ $\%)$ und bei männlichen Probanden bei $13,96( \pm 4,79) \mathrm{cm} / \mathrm{s}(65,7 \%)$. Der Unterschied zwischen den durchschnittlichen Messwerten der beiden Geschlechter erwies sich als nicht signifikant $(\mathrm{p}=0,554)$. Auch der Vergleich der Nüchterngruppen blieb ohne Signifikanz ( $\mathrm{p}=$ 0,673). Probanden mit einer Nüchternzeit von 3 h (Gruppe 1) zeigten einen durchschnittlichen Anstieg des PBF von 13,18 $( \pm 4,22) \mathrm{cm} / \mathrm{s}$. Teilnehmer mit einer Nüchternperiode von $4-8 \mathrm{~h}$ (Gruppe 2) 13,58 ( $\pm 5,67) \mathrm{cm} / \mathrm{s}$ und Probanden mit einer Nüchternzeit von $<8 \mathrm{~h}$ 
(Gruppe 3) 14,41 $( \pm 4,94) \mathrm{cm} / \mathrm{s}$. Es zeigte sich eine signifikante negative Korrelation zwischen den Ausgangswerten des PBF und der LS $(r=-0,308, p=0,002)$. Hierbei handelte es sich nach Cohen um eine mittlere Effektstärke. Zusätzlich wurde der Einfluss des Ausgangswerts des PBF auf den nahrungsbedingten Anstieg des PBF untersucht. Hierbei zeigte sich eine signifikante negative Korrelation ( $r=-0,260, p=0,009)$, wobei es sich nach Cohen um einen mittleren Effekt handelte. Der weiterführenden Regressionsanalyse zufolge sank der absolute Anstieg des PBF um -0,38 cm/s, bei einem Anstieg des Ausgangswerts des PBF um $1 \mathrm{~cm} / \mathrm{s}(\mathrm{b}=-0,38, \mathrm{p}=0,009)$. Je höher der durchschnittliche PBF vor Nahrungsaufnahme, desto geringer der durchschnittliche nahrungsbedingte Anstieg des PBF. Außerdem wurde ein möglicher Zusammenhang der nahrungsbedingten Veränderungen des PBF und der LS untersucht. Hier konnte keine signifikante Korrelation beobachtet werden ( $r=0,089, p=$ 0,380). Zudem wurde der durchschnittliche Anstieg des PBF mit den mittleren Ausgangswerten der LS korreliert. Auch hier zeigte sich kein signifikanter Zusammenhang $(r=0,034$, $\mathrm{p}=0,739)$.

\subsection{Abhängigkeit des Gallenblasenvolumens von der Nahrungsaufnahme}

Neben dem IQR-Wert und dem PBF wurde auch das GBV vor und nach Nahrungsaufnahme gemessen. Dies konnte aufgrund von zwei Teilnehmern mit stattgehabter Cholezystektomie nur bei 98 Probanden erhoben werden. Die Nüchternmessungen lagen im Mittel bei einem Volumen von 16,98 $( \pm 12,44) \mathrm{ml}$. Frauen zeigten im Durchschnitt ein geringeres GBV als Männer. Bei weiblichen Probanden lag dies bei 15,10 $( \pm 11,04) \mathrm{ml}$ und bei männlichen Probanden bei 19,01 $( \pm 13,63) \mathrm{ml}$. Mit einem $\mathrm{p}$-Wert von 0,120 ergab sich kein signifikanter geschlechtsspezifischer Unterschied. Im Gegensatz dazu zeigte der Vergleich der Nüchterngruppen signifikante Unterschiede ( $\mathrm{p}<0,001)$. Die Messwerte von Probanden der Gruppe 1 $(3 \mathrm{~h})$ unterschieden sich signifikant von denen aus der Gruppe $3(>8 \mathrm{~h})(\mathrm{p}<0,001)$. Auch Teilnehmer der Gruppe $2(4-8 \mathrm{~h})$ und der Gruppe $3(>8 \mathrm{~h})$ zeigten einen signifikanten Unterschied ( $p<0,001)$. Keine Signifikanz im Vergleich, ergab sich zwischen Gruppe 1 (3 h) und Gruppe 2 (4 - 8 h) (p = 0,961). Die im Mittel höchsten GBV wurden mit 29,29 ( \pm 10,91) $\mathrm{ml}$ in Gruppe 3 gemessen, die im Mittel niedrigsten GBV in Gruppe 1 mit 13,03 ( \pm 10,89) ml. Gruppe 2 lag mit durchschnittlich 13,69 $( \pm 10,26) \mathrm{ml}$ in der Mitte.

Nun wurden die durchschnittlichen GBV vor und nach Nahrungsaufnahme miteinander verglichen. Bei allen Teilnehmern konnte postprandial eine Abnahme des GBV beobachtet werden. Präprandial wurde im Mittel ein GBV von 16,89 $( \pm 12,44)$ ml gemessen, postprandial betrug dies 2,04 $( \pm 2,71) \mathrm{ml}$. Der Unterschied erwies sich als hochsignifikant $(\mathrm{p}<0,001)$ (Abbildung 13). 


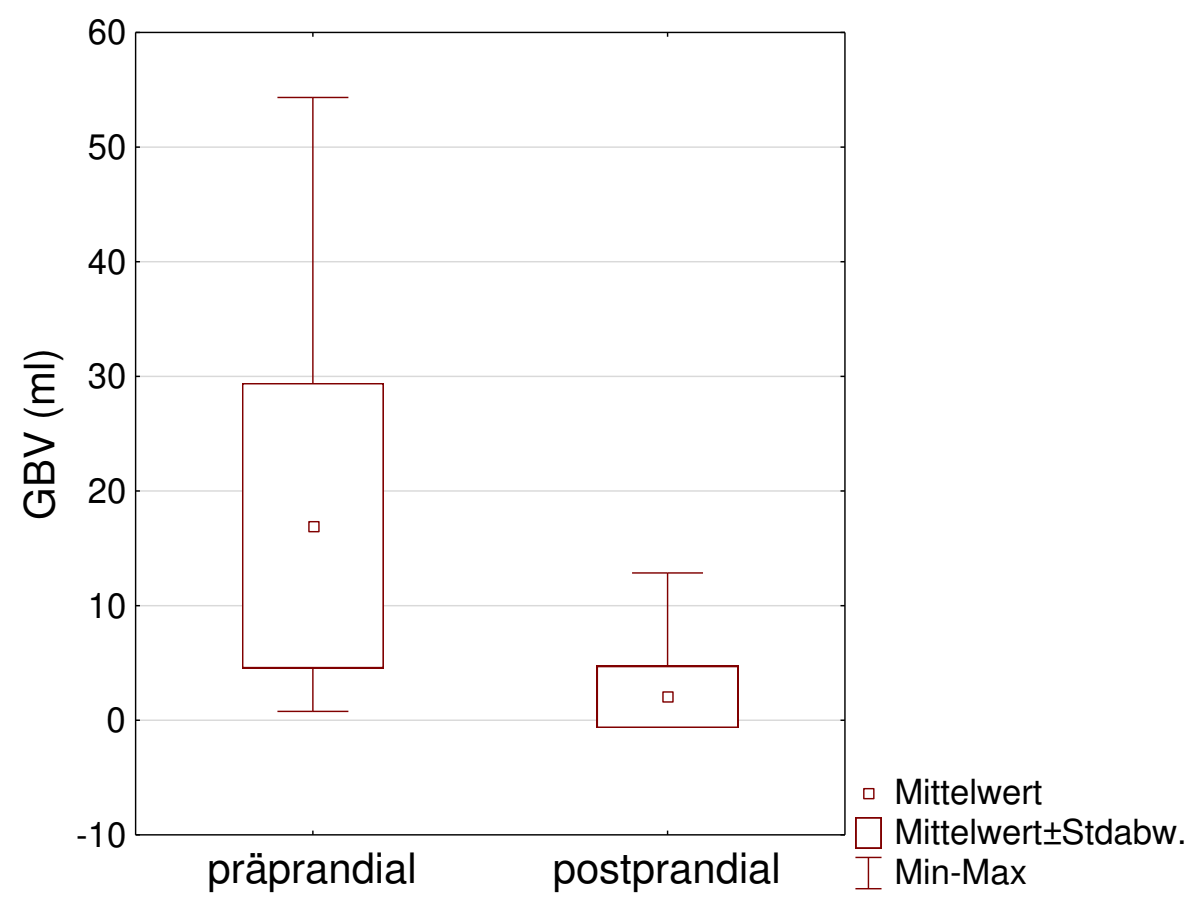

Abbildung 13: Boxplot-Diagramme der prä- und postprandialen Mittelwerte des GBV in $\mathrm{ml}(\mathrm{p}<$ $0,001, \mathrm{n}=100)$.

Auch hier wurde die Abhängigkeit der nahrungsbedingten Änderung des GBV von anderen Einflussfaktoren untersucht, indem für jeden Probanden die Differenz zwischen den beiden GBV Messungen (prä- und postprandial) ermittelt wurde. Im Durchschnitt ergab sich eine Abnahme des GBV nach Nahrungsaufnahme von 14,64 ( $\pm 10,75) \mathrm{ml}(-89,2 \%)$ (Tabelle 12).

Tabelle 12: durchschnittliches GBV $( \pm \mathrm{SD})$ und Minimum bis Maximum prä- und postprandial und die durchschnittliche postprandiale Abnahme des GBV in $\mathrm{ml}(\mathrm{n}=100)$.

\begin{tabular}{l|l|l|l} 
& präprandial & postprandial & GBV Abnahme \\
\hline GBV $(\mathrm{ml})$ & $16,98( \pm 12,44)$ & $2,04( \pm 2,71)$ & $14,64( \pm 10,75)$ \\
\hline $\begin{array}{l}\text { GBV Minimum - Ma- } \\
\text { ximum }(\mathrm{ml})\end{array}$ & $0,77-54,33$ & $0,04-12,85$ & $0,61-44,96$
\end{tabular}

Bei Frauen wurde im Mittel eine Abnahme des GBV von 13,20 ( $\pm 9,53) \mathrm{ml}(-88,2 \%)$ gemessen, bei Männern betrug dies durchschnittlich 16,83 $( \pm 11,57) \mathrm{ml}(-90,4 \%)$. Der Vergleich der Geschlechter blieb ohne Signifikanz ( $p=0,092)$. Bezüglich der Nüchterngruppen zeigte sich auch bei der nahrungsbedingten GBV Abnahme ein signifikanter Effekt ( $\mathrm{p}<$ 0,001). Teilnehmer die $>8 \mathrm{~h}$ nüchtern blieben, zeigten im Durchschnitt eine stärkere nahrungsbedingte Abnahme des GBV als Probanden der anderen beiden Nüchterngruppen. Probanden in Gruppe $1(3 \mathrm{~h})$ zeigten durchschnittlich eine Abnahme des GBV von 12,11 ( \pm $10,33) \mathrm{ml}(-91,4 \%)$. In Gruppe $2(4-8 \mathrm{~h})$ von 12,04 ( \pm 8,57) $\mathrm{ml}(-89,5 \%)$ und in Gruppe 3 
$(>8 \mathrm{~h})$ von $24,86( \pm 9,14) \mathrm{ml}(-85,4 \%)$. Sowohl zwischen Gruppe 1 und 3, als auch zwischen Gruppe 2 und 3 wurden signifikante Unterschiede beobachtet $(p<0,001, p<0,001)$. Der Vergleich von Probanden der Gruppen 1 und 2 zeigte keine Signifikanz $(p=0,999)$ (Abbildung 14, Tabelle 13).

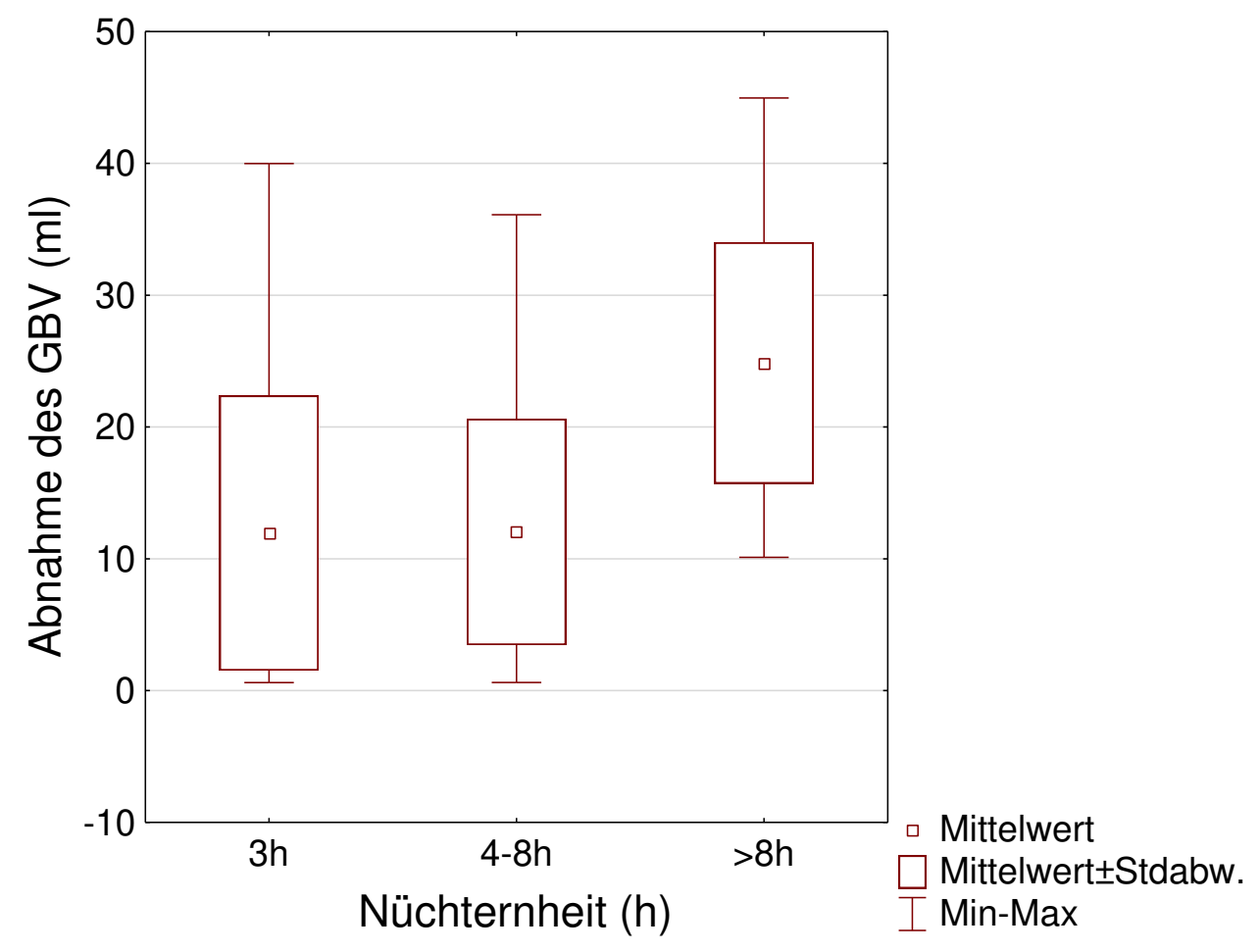

Abbildung 14: Boxplot-Diagramme der postprandialen Abnahme des GBV gruppiert nach Nüchternheit in h (Gruppe 1 (3 h), n= 32; Gruppe 2 (4 - 8 h), $n=45$; Gruppe $3(>8 h), n=23)$.

Tabelle 13: $\mathrm{p}$-Werte der durchschnittlichen postprandialen Abnahme des GBV für verschiedene Nüchterngruppen (Gruppe 1 ( 3 h), $n=32$; Gruppe 2 ( 4 - 8 h), n = 45; Gruppe 3 (> 8 h), n = 23).

\begin{tabular}{l|l|l|l} 
Nüchterheit & Gruppe 1 $(3 \mathrm{~h})$ & Gruppe 2 $(4-8 \mathrm{~h})$ & Gruppe 3 $(>8 \mathrm{~h})$ \\
\hline Gruppe 1 $(3 \mathrm{~h})$ & & 0,999 & $<0,001$ \\
\hline Gruppe 2 $(4-8 \mathrm{~h})$ & 0,999 & & $<0,001$ \\
\hline Gruppe 3 $(>8 \mathrm{~h})$ & $<0,001$ & $<0,001$ &
\end{tabular}

Die durchschnittlichen Messwerte der nahrungsbedingten Abnahme des GBV und der Ausgangswerte des GBV korrelierten signifikant positiv $(r=0,984, p<0,001)$. Hierbei handelte es sich nach Cohen um einen starken Effekt. Eine signifikante Korrelation zwischen den durchschnittlichen nahrungsbedingten Abnahmen des GBV und den mittleren Ausgangswerten der LS wurde nicht beobachtet $(r=-0,153, p=0,132)$. Ebenfalls keinen signifikanten Effekt ergab die Korrelation der nahrungsbedingten Abnahme des GBV und des Anstiegs der LS $(r=0,156, p=0,126)$. Die Korrelation der durchschnittlichen nahrungsbedingten 
Veränderungen des GBV und des PBF erwies sich als signifikant positiv ( $r=0,209, p=$ 0,039). Nach Cohen handelte es sich hierbei um einen schwachen Effekt. 


\section{Diskussion}

Seit einigen Jahren spielt die Elastographie eine immer wichtigere Rolle in der nicht-invasiven Bestimmung des Fibrosegrades der Leber und hat bereits Einzug in einige Leitlinien gefunden. Unter anderem aufgrund des direkten Einflusses auf das Erkrankungsmanagement ist es besonders wichtig, Kenntnis über mögliche Einflussfaktoren zu haben. Die vorliegende Arbeit untersucht den Einfluss der Nahrungsaufnahme auf die LSM an lebergesunden Probanden mit der 2D-SWE (LOGIQ E9). Neben diesem zentralen Aspekt wurde auch der Einfluss der Parameter Geschlecht, Alter, BMI, Alkoholkonsum, Medikamenteneinnahme und Nüchternperiode auf die LSM untersucht. Zunächst soll kurz auf diese Einflussgrößen und auf die in dieser Studie erhobenen präprandialen LS-Werte eingegangen werden. Im Anschluss wird die Abhängigkeit der LS von der Nahrungsaufnahme ausführlich diskutiert.

\subsection{Lebersteifigkeit (2D-SWE) bei lebergesunden Probanden}

Die Normwerte der 2D-SWE für das LOGIQ E9 für lebergesunde Probanden lagen bei durchschnittlich 4,80 $( \pm 0,94) \mathrm{kPa}$. Alle Messungen lagen in einem Bereich zwischen 2,66 und 7,02 kPa. Diese Ergebnisse sind vergleichbar mit denen von Serra et al. Diese Arbeitsgruppe publizierte Normwerte von 4,65 $( \pm 1,15) \mathrm{kPa}$ (Serra et al. 2018). Auch Bende et al. zeigten ähnlich Messergebnisse für lebergesunde Probanden $(5,1( \pm 1,3) \mathrm{kPa})$ (Bende et al. 2018). Beide Studien nutzen ebenfalls das von uns verwendete Gerät LOGIQ E9.

In der vorliegenden Arbeit wiesen männliche Probanden eine signifikant höhere LS auf als weibliche Probanden $(5,09( \pm 0,95) \mathrm{kPa}$ vs. 4,80 $( \pm 0,94) \mathrm{kPa})$. Diesen geschlechtsspezifischen Unterschied der LS beobachteten bereits andere Studien, sowohl für die TE (Colombo et al. 2011) als auch für die p-SWE (Liao et al. 2015). Huang et al. zeigten dies ebenfalls für die 2D-SWE anhand des Aixplorers (Huang et al. 2014), und auch für das von uns verwendete Gerät LOGIQ E9 wurde dieser geschlechtsspezifischer Unterschied bereits festgestellt (Bende et al. 2018; Serra et al. 2018). Im Gegensatz dazu stehen Arbeiten von Madhok et al. und Horster et al., die keinen Einfluss des Geschlechts auf die LS feststellen konnten (Horster et al. 2010; Madhok et al. 2013). Eine mögliche Erklärung für diese Diskrepanz ist, dass die beiden Studien mit der p-SWE und nicht mit der von uns verwendeten 2D-SWE durchgeführt wurden.

Bezüglich des Alters konnten wir keinen Einfluss auf die LSM feststellen. Dieses Ergebnis deckt sich mit denen anderer Studien (Kim et al. 2010; Popescu et al. 2011; Huang et al. 2014). Hier ist darauf hinzuweisen, dass das durchschnittliche Alter unseres Kollektivs bei 25,8 \pm 6,1 Jahren lag und somit nur eine eingeschränkte Aussage über Patienten höheren Alters getroffen werden kann. Dies stellt eine Limitation der vorgelegten Arbeit dar.

Der BMI zeigte, vergleichbar mit anderen Studien (Kim et al. 2010; Popescu et al. 2011; Son et al. 2012; Sirli et al. 2013; Huang et al. 2014), ebenfalls keinen Einfluss auf die LS. 
Auch hier ist die Aussagekraft für höhere BMI-Klassen eingeschränkt, da der mittlere BMI des Kollektivs bei $22,43 \pm 2,37 \mathrm{~kg} / \mathrm{m}^{2}$ lag und somit vorwiegend junge und schlanke Probanden eingeschlossen wurden.

Die Einnahme von Medikamenten erwies sich ebenfalls nicht als Einflussfaktor der LS. Hier ist zu erwähnen, dass die Gruppe der Teilnehmer mit Medikamenteneinnahme deutlich kleiner war $(n=30)$ als die Gruppe der Probanden ohne Medikamenteneinnahme ( $n=70)$. Außerdem lag bezüglich der Medikamenteneinnahme eine ungleiche Geschlechterverteilung vor $(\mathrm{w}=26, \mathrm{~m}=4)$. Zudem wurden die angegeben Präparate aufgrund unterschiedlicher medizinischer Indikationen eingenommen (Verhütung, Behandlung von diversen Erkrankungen: Depressionen, Hypothyreose, Hypertonie, Infektion der Atemwege, Colitis ulzerosa).

Die Untersuchung des durchschnittlichen täglichen Alkoholkonsums als Einflussfaktor zeigte keine Signifikanz. Die im Mittel höchsten LS-Werte erreichten Probanden mit einem Alkoholkonsum von $20-30 \mathrm{~g} / \mathrm{d}$. Zu beachten ist, dass bezogen auf die fünf Alkoholkonsumkategorien ungleiche Gruppengrößen vorlagen (Kategorie 1: $\mathrm{n}=26$, Kategorie 2: $\mathrm{n}=$ 50, Kategorie 3: $n=20$, Kategorie 4: $n=4$ ). Außerdem lag der angegebene Alkoholkonsum aller Probanden im gesundheitlich niedrigen Risikobereich (Wood et al. 2018). Eine Verifizierung durch biochemische Marker wie z. B. das mittlere Erythrozyten-Volumen (MCV), das carbohydrat deficient transferrin (CDT) oder Alkoholblutspiegelmessungen wurde in dieser Arbeit jedoch nicht durchgeführt, sondern sich auf die Aussagen des Fragebogens verlassen.

\subsection{Abhängigkeit der Lebersteifigkeit (2D-SWE) von der Nahrungsaufnahme}

Wie oben erwähnt, beschäftigte sich die vorliegende Studie vor allem mit der Fragstellung, ob die Nahrungsaufnahme die LSM beeinflusst. Um diesen Einfluss zu untersuchen, wurden 100 freiwillige lebergesunde Probanden mit einer Nüchternperiode von mindestens 3 h, vor und 30 bis 40 min nach Einnahme einer standardisierten 800-kcal-Mahlzeit untersucht. Bei der Analyse des Einflusses der Nüchternperiode konnte ein Trend beobachtet werden. Die niedrigsten LS-Ausgangswerte wurden bei Probanden mit einer Nüchternperiode von $>8 \mathrm{~h}$ beobachtet, darauf folgten Probanden mit einer Nahrungskarenz von $4-8$ h. Die höchsten LS-Ausgangswerte zeigten Probanden, die $3 \mathrm{~h}$ gefastet hatten. Das bedeutet, je länger die Probanden gefastet hatten, desto niedriger war der durchschnittlich gemessene LS-Ausgangswert. Allerdings blieb der Vergleich ohne Signifikanz.

Andere Studien mit der TE kamen zu dem Ergebnis, dass die LS 2 h nach Nahrungsaufnahme wieder auf den LS-Ausgangswert gesunken ist (Arena et al. 2013; Alvarez et al. 2015). Für die 2D-SWE zeigten Kjaergaard et al., dass bei über $24 \%$ der Probanden auch $3 \mathrm{~h}$ nach Einnahme der Mahlzeit weiterhin erhöhte LS-Werte gemessen wurden (Kjærgaard et al. 
2017). Diese Daten geben Anlass zu der Annahme, dass eine Nüchternperiode von mehr als $3 \mathrm{~h}$ vor der Messung der LS anzustreben ist.

Im Vergleich der prä- und postprandialen LS-Werte der vorliegenden Studie konnte bei 94 $\%$ der Teilnehmer ein Anstieg der LS beobachtet werden.

Den Berechnungen zufolge stellt die Nahrungsaufnahme einen signifikanten Einflussfaktor der LS dar. Der nahrungsbedingte LSA betrug im Mittel 0,93 $( \pm 0,74) \mathrm{kPa}$. Dies entsprach einem Anstieg von 21,6\%.

Ein signifikanter LSA nach Nahrungsaufnahme wurde für die TE bereits in einigen Studien beobachtet. Die erste Studie wurde im Jahr 2009 von Mederacke et al. veröffentlicht. Es wurde eine Gruppe von 64 Personen mit Hepatitis-C-Virus-Infektion (HCV) vor und nach Einnahme einer 600-kcal-Mahlzeit untersucht. Es zeigte sich eine signifikante Erhöhung der Lebersteifigkeitswerte, mit einem Maximum $30 \mathrm{~min}$ nach Beendigung der Mahlzeit (Mederacke et al. 2009). Auch Arena et al. zeigten ähnliche Ergebnisse bei Patienten mit HCV-Infektion (Arena et al. 2013). Außerdem wurde eine Erhöhung der Messwerte nach Nahrungsaufnahme, unabhängig von der Ätiologie der zugrundeliegenden Zirrhose, beobachtet (Berzigotti et al. 2013). Lemoine et al. beobachteten einen LSA nach Nahrungsaufnahme ebenfalls für lebergesunde Probanden (Lemoine et al. 2014). Weitere Studien bestätigten den nahrungsbedingten LSA für die TE, vor allem jedoch für Patienten mit chronischen Lebererkrankungen (Alvarez et al. 2015; Ratchatasettakul et al. 2017).

Auch für die p-SWE wurde der Einfluss der Nahrungsaufnahme auf die LS bei gesunden Probanden nachgewiesen. Popescu et al. zeigten eine Erhöhung der LS 60 min nach Nahrungsaufnahme um $15 \%$ des Ausgangswerts. Die Messungen 3 h nach Nahrungsaufnahme, verglichen mit den Ausgangswerten, waren weiterhin erhöht, allerdings ohne statistische Signifikanz (Popescu et al. 2013). Goertz et al. beschrieben dagegen lediglich eine nahrungsbedingten LSA von ca. $9 \%$ (Goertz et al. 2012).

Gersak et al. und Kjaergaard et al. bestätigten diese Annahme ebenfalls für die 2D-SWE anhand des Aixplorers (Gersak et al. 2016a; Kjærgaard et al. 2017). Kjaergaard et al. untersuchten 64 Patienten mit Fibrose bzw. Zirrhose. Alle Teilnehmer fasteten für 3 h. Ein durchschnittlicher LSA von $22 \%$ wurde für eine 625-kcal-Mahlzeit beobachtet. Bei der Einnahme einer 1250-kcal-Mahlzeit zeigte sich ein LSA von $31 \%$ (Kjærgaard et al. 2017). Gersak et al. untersuchten lebergesunde Probanden. Vor Einnahme einer 748-kcal-Mahlzeit nahmen die Teilnehmer $6 \mathrm{~h}$ keine Nahrung zu sich. Die postprandialen Messungen ergaben im Mittel einen signifikanten LSA von 2,2\% (Gersak et al. 2016a).

Die existierenden Daten anderer Elastographie Methoden stimmen daher mit unseren Daten der 2D-SWE (GE LOGIQ E9) überein und zeigen, dass die Nahrungsaufnahme die LSM signifikant beeinflusst. Allerdings wurden auch Diskrepanzen in den prozentualen Veränderungen der LS aufgezeigt. Popescu et al. zeigten einen nahrungsbedingten LSA von $15 \%$, Goertz et al. von $9 \%$, Gersak et al. von 2,2\% und Kjaergaard et al. von $22 \%$ bzw. $31 \%$. 
Der durchschnittliche LSA betrug laut unseren Ergebnissen 21,6 \% und deckt sich somit am ehesten mit den Ergebnissen von Kjaergaard et al., welche ebenfalls die 2D-SWE nutzen. $\mathrm{Zu}$ erwähnen ist allerdings, dass diese Arbeit ausschließlich Patienten mit Leberfibrose untersuchte (Kjærgaard et al. 2017). Die Abweichungen zu den Ergebnissen von Popescu et al. und von Goertz et al. lassen sich möglicherweise damit erklären, dass diese beiden Studien die p-SWE und nicht die von uns verwendeten 2D-SWE nutzten. Außerdem wurden die LSM bei Popescu et al. erst 60 min nach Einnahme der Mahlzeit durchgeführt, verglichen mit 30 - 40 min in der vorliegenden Studie. Bei Popescu et al. fehlte eine exakte Angabe des Energiegehaltes, jeder Proband nahm ein 200 g Sandwich ein (Popescu et al. 2016).

Die Diskrepanz zu den Ergebnissen von Gersak et al. (2D-SWE) lässt sich möglicherweise durch die unterschiedlichen Stichprobengrößen erklären, denn Gersak et al. untersuchten lediglich 31 Probanden (Gersak et al. 2016a). Die vorliegende Studie schloss 100 Probanden in die Analyse ein. Ein weiterer Erklärungsversuch könnten die unterschiedlichen Energiegehalte der Nahrung darstellen. Allerdings konnte von Kjaergaard et al. weder für die LSAusgangswerte noch für den LSA signifikante Unterschiede im Vergleich einer Mahlzeit mit $625 \mathrm{kcal}$ und einer mit $1250 \mathrm{kcal}$ nachgewiesen werden (Kjærgaard et al. 2017). Dementsprechend scheint die Größe der Mahlzeit die LS und den LSA nicht zu beeinflussen, wenn ein Energiegehalt von mindestens $625 \mathrm{kcal}$ vorausgesetzt werden kann. Dies ist für unsere Studie zutreffend, denn jeder Teilnehmer nahm eine 800-kcal-Mahlzeit ein.

Auch wenn sich unsere Ergebnisse teilweise von den erwähnten Studien unterscheiden, kamen sowohl die Arbeiten der TE als auch der p-SWE und der 2D-SWE zu dem Ergebnis, dass die Nahrungsaufnahme die LS signifikant beeinflusst.

Auch die Tatsache, dass verglichen mit den Messwerten der TE, Lebergesunde mit dem LOGIQ E9 höhere LS-Werte und Leberkranke niedrigere LS-Werte aufweisen (Bende et al. 2017; Bende et al. 2018), scheint keinen Einfluss auf den Zusammenhang des LSA nach Nahrungsaufnahme zu haben. Keine der oben genannten Arbeiten zum Einfluss der Nahrungsaufnahme verwendete allerdings das Gerät LOGIQ E9.

Die einzige Studie, die dieses Gerät verwendete zeigte konträre Ergebnisse zu unseren Daten. Popescu et al. untersuchten 33 gesunde Probanden vor und 30 min, $1 \mathrm{~h}$ und $2 \mathrm{~h}$ nach Einnahme einer Mahlzeit. Der Vergleich der durchschnittlichen LSM vor und nach Nahrungsaufnahme blieb ohne Signifikanz (Popescu et al. 2016). Eine mögliche Ursache für die konträren Ergebnisse könnte die begrenzte Stichprobengröße darstellen. Es wurden nur 33 Probanden untersucht. Außerdem liegt keine Information über die Mahlzeitgröße (kcal) vor, da diese Studie nur als Abstract veröffentlicht wurde, was die Aussagekraft ebenfalls einschränkt. 


\subsubsection{Einfluss des LS-Ausgangswerts auf den postprandialen LSA}

Interessante Ergebnisse ergaben sich bei der Betrachtung des Einflusses des LS-Ausgangswerts auf den nahrungsbedingten LSA. Bei Patienten mit einem höheren LS-Ausgangswert wurden postprandial geringere LSA beobachtet als bei Patienten mit einem niedrigeren LSAusgangswert. Auch Mederacke et al. machten ähnliche Beobachtungen. Patienten bei denen nüchtern eine LS von über $10 \mathrm{kPa}$ gemessen wurde, zeigten keinen signifikanten präund postprandialen Unterschied der LS (Mederacke et al. 2009). Eine mögliche Ursache könnte einerseits darin zu finden sein, dass die LS bei höheren Ausgangswerten nicht so stark von der Nahrungsaufnahme beeinflusst wird, wie bei niedrigeren Ausgangswerten. Andererseits wäre es auch möglich, dass der LSA bei den erstgenannten langsamer erfolgt, da bei Patienten mit Leberfibrose bzw. Zirrhose hämodynamische Veränderungen des Blutflusses nach Nahrungsaufnahme langsamer erfolgen als bei lebergesunden Probanden (Ludwig et al. 1998). Wobei zumindest für die Studie von Mederacke et al. die LS von Patienten mit Lebererkrankungen zu mehreren aufeinanderfolgenden Zeitpunkten nach Nahrungsaufnahme gemessen wurde ( 0 - 180 min nach Einnahme der Mahlzeit). Auch nach 180 min war der LSA bei Patienten mit einem LS-Ausgangswert von über $10 \mathrm{kPa}$ nicht signifikant (Mederacke et al. 2009). Wichtig zu erwähnen ist, dass Mederacke et al. im Gegensatz zu der vorliegenden Arbeit ausschließlich Patienten mit Lebererkrankungen untersuchten und dies anhand der TE.

Im Gegensatz zu Mederacke et al. zeigten Arena et al. und Alvarez et al. einen signifikanten LSA für alle Fibrosestadien anhand der TE. In den beiden genannten Studien war der absolute Anstieg der LS nach Nahrungsaufnahme sogar höher bei Studienteilnehmern mit hochgradiger Fibrose als bei Probanden mit milder bzw. bei Probanden ohne Fibrose (Arena et al. 2013; Alvarez et al. 2015).

Die weitere Analyse dieses Zusammenhangs sollte durch nachfolgende Studien erfolgen, denn die vorgelegten Ergebnisse werfen die Frage auf, inwiefern die Nahrungsaufnahme bei Patienten mit erhöhten LS-Werten bzw. Patienten mit Leberfibrose zu berücksichtigen oder sogar zu vernachlässigen ist.

\subsubsection{Einfluss des Geschlechts, des Alters, des BMIs, des Alkoholkonsums, der Medikamenteneinnahme und der Nüchternperiode auf den postprandialen LSA}

Um den Einfluss der Nahrungsaufnahme auf die LS detaillierter zu analysieren, wurde die Abhängigkeit des nahrungsbedingten LSA von potenziellen Einflussgrößen geprüft. Ein signifikant geschlechtsspezifischer Effekt wie auf den Ausgangswert der LS, welcher oben beschrieben wurde (s. 4.1), konnte bezogen auf den nahrungsbedingten LSA nicht gezeigt werden. Bei weiblichen Probanden stieg die LS postprandial durchschnittlich um 0,87 $( \pm 0,68)$ $\mathrm{kPa}(20,6 \%)$ und bei männlichen Probanden um 1,00 $( \pm 0,80) \mathrm{kPa}(22,7 \%)$. Somit ergaben 
sich ähnliche Ergebnisse wie in der Studie von Lemoine et al. (Lemoine et al. 2014). Hier konnte ebenfalls keine geschlechtsspezifische Abhängigkeit des nahrungsbedingten LSA feststellt werden (Lemoine et al. 2014). Im Gegensatz dazu beobachteten Gersak et al. einen signifikanten Unterschied zwischen den Geschlechtern bei gesunden Probanden (Gersak et al. 2016a). Männer zeigten einen LSA von 6,1 \% und Frauen von 2,3 \% (Gersak et al. 2016a). Auch hier lässt sich die Größe der Stichprobe (31 vs. 100 Probanden) als mögliche Ursache der Diskrepanz in den Ergebnissen aufführen.

Der Parameter Alter zeigte keinen Einfluss auf den LSA nach Nahrungsaufnahme. Zu der gleichen Erkenntnis kamen auch Lemoine et al. (Lemoine et al. 2014).

Bei der Untersuchung des Einflusses des BMIs auf den postprandialen LSA zeigten sich interessante Ergebnisse. Für Probanden mit einem höheren BMI konnte ein stärkerer LSA nachgewiesen werden als für Probanden mit einem niedrigeren BMI. $\mathrm{Zu}$ ähnlichen Ergebnissen kamen auch Popescu et al. Hier zeigten Studienteilnehmer mit niedrigerem BMI ebenfalls signifikant geringere Veränderungen in der LS nach Nahrungsaufnahme (Popescu et al. 2013). Andere Arbeitsgruppen kamen allerdings zu gegensätzlichen Ergebnissen. Lemoine et al. konnten keinen Einfluss des BMIs feststellen, genauso wenig wie Gersak et al. (Lemoine et al. 2014; Gersak et al. 2016a).

Eine mögliche Erklärung für den von uns beobachteten Effekt könnte die Dynamik des Leberblutflusses darstellen. Es wäre möglich, dass Veränderungen der Hämodynamik in unterschiedlichen BMI Klassen zu anderen Zeitpunkten nach Nahrungsaufnahme eintreten (Popescu et al. 2013). Da die LS nur einmal 30 - 40 min nach Einnahme der Mahlzeit gemessen wurde, ist es nicht möglich hierzu eine Aussage zu treffen. Außerdem ist zu erwähnen, dass der durchschnittliche BMI der gesunden Probanden im normalgewichtigen Bereich lag. Weitere Untersuchungen dieses Zusammenhangs könnte Gegenstand folgender Studien werden.

Bezüglich der Einnahme von Medikamenten und auch bezüglich des durchschnittlichen Alkoholkonsums wurde kein Einfluss auf den Anstieg der LS nach Nahrungsaufnahme beobachtet. Auch hier muss einschränkend erwähnt werden, dass die untersuchte Population von jungen und gesunden Probanden nur sehr wenige Medikamente einnahm.

Auch die Länge der Nüchternperiode zeigte keinen signifikanten Einfluss auf den postprandialen LSA. Allerdings konnte hier ein statistischer Trend beobachtet werden. Der nahrungsbedingte LSA war umso höher, je länger die Probanden vor der Untersuchung gefastet hatten.

\subsection{Abhängigkeit des Pfortaderblutflusses von der Nahrungsaufnahme}

Ein möglicher Erklärungsversuch für den postprandialen LSA könnten nahrungsbedingte Veränderungen des hepatischen Blutflusses darstellen. In diversen Studien wurde gezeigt, 
dass die hepatische Zirkulation und der hepatische Blutfluss nach Einnahme einer Mahlzeit ansteigen. Dies traf sowohl auf lebergesunde, als auch auf leberkranke Patienten zu (Moneta et al. 1988; Gaiani et al. 1989; Sabbá et al. 1991; Dauzat et al. 1994; Schiedermaier et al. 2006; Zardi et al. 2008). Um den vermuteten Zusammenhang mit dem LSA und den Änderungen der hepatischen Blutzirkulation zu analysieren, wurde in der vorliegenden Studie der PBF vor und 30 - 40 min nach Nahrungsaufnahme gemessen. Wie zuvor vermutet, stieg der PBF in $100 \%$ der Messungen postprandial signifikant an.

Interessanterweise korrelierten die nahrungsbedingten Veränderungen der LS und des PBF nicht signifikant miteinander. Diese Erkenntnis deckt sich mit Beobachtungen anderer Studien. Kjaergaard et al. und Alvarez et al. untersuchten diesen Zusammenhang mittels der TE. Es wurde ein Anstieg des PBF in Abhängigkeit von der Nahrungsaufnahme festgestellt. Zudem wurde ebenso ein nahrungsbedingter Anstieg der LS beobachtet. Es konnte allerdings in keiner der beiden genannten Studien eine signifikante Korrelation zwischen dem Anstieg der LS und dem Anstieg des PBF nachgewiesen werden (Alvarez et al. 2015; Kjærgaard et al. 2017). Im Gegensatz dazu zeigte eine andere Studie einen linearen Zusammenhang zwischen den postprandial Veränderungen der LS und des PBF (Barone et al. 2015). Diese Arbeit beruft sich auf Daten der TE.

Ein signifikanter Unterschied in den Messungen des PBF bezüglich der unterschiedlich langen Fastenzeiten konnte nicht nachgewiesen werden. Es ergab sich aber ein signifikanter Zusammenhang zwischen den präprandialen Messergebnissen des PBF und dem nahrungsbedingten Anstieg des PBF. In Analogie mit dem LSA zeigte sich, dass je höher der durchschnittliche PBF vor Nahrungsaufnahme war, desto geringer der nahrungsbedingte Anstieg des PBF ausfiel.

Den Beobachtungen zufolge scheint es nicht möglich zu sein, den nahrungsbedingten Anstieg der LS allein durch Veränderungen des PBF zu erklären. Wahrscheinlich ist der Anstieg der LS nach Einnahme einer Mahlzeit multifaktoriell bedingt. Möglicherweise spielen Variationen des arteriellen Blutflusses und/oder der Zirkulation der Lebervenen eine entscheidende Rolle. Außerdem sind Veränderungen der LS gemäß der sinusoidal-pressureHypothese mit intrahepatischen Druckänderungen assoziiert (Mueller 2016), worauf die Einnahme einer Mahlzeit ebenfalls Einfluss nehmen könnte. Zudem steigt der resistance index der Arteria hepatica nach Nahrungsaufnahme an. Dies könnte, neben der Erhöhung des PBF, eine entscheidende Erklärung für einen Anstieg der LS nach Nahrungsaufnahme sein, obwohl die Geschwindigkeit des Blutflusses in der Arteria hepatica postprandial abnimmt (Numata et al. 1999).

Dies wurde jedoch in der vorliegenden Arbeit nicht untersucht und auch in der Literatur finden sich diesbezüglich bislang keine Daten. 


\subsection{Abhängigkeit des IQR-Werts von der Nahrungsaufnahme}

Der IQR-Wert stellt ein Kriterium zur Beurteilung der Qualität der Messergebnisse dar. Gemäß den Qualitätskriterien des Herstellers wurden nur LSM mit einem IQR-Wert < $30 \%$ des Medians als valide anerkannt. In der vorliegenden Arbeit traf dies auf $100 \%$ der Messungen zu, was eine gute Messqualität beweist. Der durchschnittliche IQR-Wert vor Nahrungsaufnahme lag bei $0,51( \pm 0,21) \mathrm{kPa}$, was $10,6 \%$ des durchschnittlichen Medians entspricht. Nach Nahrungsaufnahme betrug der IQR-Wert im Mittel 0,54 $( \pm 0,22) \mathrm{kPa}(9,4 \%$ des durchschnittlichen Medians). Die Nahrungsaufnahme beeinflusst folglich die Validität der Messungen nicht.

Erwähnenswert ist die Beobachtung eines geschlechtsspezifischen Unterschieds bei den präprandialen Messungen. Weibliche Probanden zeigten vor Nahrungsaufnahme signifikant niedrigere IQR-Werte $(0,46( \pm 0,21) \mathrm{kPa})$ als männliche Probanden $(0,51( \pm 0,21) \mathrm{kPa})$. Nach derzeitigen Kenntnissen ist diese Studie die erste, die diesen Effekt beobachtet hat. Eine mögliche Erklärung könnte darin liegen, dass bei Männern ebenfalls signifikant höhere LS gemessen wurden als bei Frauen.

Die Länge der Nüchternperiode hat sich jedoch nicht auf die Validität der Messungen ausgewirkt.

Lemoine et al. kamen zu gegensätzlichen Ergebnissen. Sie beobachteten sowohl bei leberkranken als auch bei lebergesunden Probanden postprandial einen signifikanten Anstieg der IQR-Werte. Wobei im Mittel höhere IQR-Werte gemessen wurden als bei den von uns durchgeführten Messungen (Lemoine et al. 2014). Dies könnte einen möglichen Erklärungsversuch für die Abweichung der Beobachtungen darstellen. Zudem wurden die LSM mittels der TE durchgeführt. Dies gibt Anlass zu der Annahme, dass die 2D-SWE, bezogen auf die Validität der LSM, der TE möglicherweise überlegen ist.

\subsection{Abhängigkeit des Gallenblasenvolumens von der Nahrungsaufnahme}

Das GBV vor Nahrungsaufnahme lag durchschnittlich bei $16,98( \pm 12,44) \mathrm{ml}$. Ein geschlechtsspezifischer Unterschied wurde nicht beobachtet. Allerdings scheint die Länge der Nüchternperiode eine Rolle zu spielen. Probanden, die über $8 \mathrm{~h}$ fasteten, zeigten im Durchschnitt signifikant höhere GBV als Probanden die $4-8$ h oder 3 h nüchtern waren.

Im Mittel konnte eine Abnahme des GBV nach Nahrungsaufnahme von 14,64 $( \pm 10,75) \mathrm{ml}$ beobachtet werden. Die entspricht einer Abnahme von 89,2\%. 
Bereits anhand einer anderen Studie wurde eine signifikante Abnahme des GBV 45 min nach Einnahme einer 554-kcal-Mahlzeit nachgewiesen. Dieser Effekt wurde sowohl an 50 Patienten mit NAFLD als auch an einer 38 Probanden großen Kontrollgruppe beobachtet (Colak et al. 2016).

Die von uns beobachtete nahrungsbedingte Abnahme des GBV beweist, dass die Nahrungsaufnahme in $100 \%$ der Probanden erfolgreich gewesen ist.

Bezogen auf die Abnahme des GBV von 89,2 \% zeigte sich kein geschlechtsspezifischer Unterschied. Allerdings beeinflusste die Länge der Fastenperiode die nahrungsbedingte Abnahme des GBV. Bei Studienteilnehmern, die über $8 \mathrm{~h}$ keine Nahrung zu sich genommen hatten, wurde die deutlichste Abnahme beobachtet. Zudem wurde nachgewiesen, dass je höher das gemessene GBV vor Nahrungsaufnahme, desto größer die Abnahme nach Nahrungsaufnahme. Ein Zusammenhang zwischen Veränderungen der LS und des GBV konnte nicht beobachtet werden.

\subsection{Limitationen der Arbeit}

Eine Limitation der vorliegenden Arbeit ist das Fehlen einer Kontrollgruppe mit Probanden, bei denen nach mindestens $3 \mathrm{~h}$ Nahrungskarenz die erste LSM erfolgte und eine erneute Messung nach 30-40 min - ohne die Einnahme einer Mahlzeit - stattfand. Auch Goertz et al. und Gersak et al. verzichteten auf eine solche Kontrollgruppe (Goertz et al. 2012; Gersak et al. 2016a). Wobei Arena et al. und Popescu et al. bereits anhand oben beschriebener Kontrollen nachweisen konnten, dass es ohne Nahrungsaufnahme bzw. durch das Trinken von Wasser zu keinen signifikanten Veränderungen der LS kam (Arena et al. 2013; Popescu et al. 2013).

$\mathrm{Zu}$ erwähnen ist, dass die vorliegende Studie, im Gegensatz zu Goertz et al., die Kalorienanzahl nicht geschlechts- und gewichtsadaptiert berechnet hat (Goertz et al. 2012). Außerdem muss berücksichtigt werden, dass sowohl bei der vorliegenden Studie als auch in dem Großteil der anderen genannten Arbeiten eine hochkalorische Flüssigmahlzeit verwendet wurde. Dies entspricht nicht einer realitätsgetreuen Ernährung. Jedoch wurde durch die Verwendung der Fresubin-Trinkflaschen gewährleistet, möglichst standardisierte Untersuchungsbedingungen zu schaffen.

Eine weitere Limitation stellt, wie auch bei Popescu et al. und Gersak et al., der Verzicht auf Blutuntersuchungen zum Ausschluss von Lebererkrankungen dar. In dem von den Probanden auszufüllenden Fragebogen wurde angegeben, ob eine bereits diagnostizierte Lebererkrankung vorliegt oder nicht. Trotzdem ist nicht auszuschließen, dass auch Teilnehmer mit bisher unbekannten Lebererkrankungen in die Studie miteingeschlossen wurden. Die BBild-sonographische Untersuchung der Leber half allerdings dabei, Pathologien der Leber auszuschließen. 
Vor Beginn der Studie fand keine Evaluation der Intra- oder Interbeobachtervariabilität statt. Diese wurde allerdings bereits anhand anderer Studien untersucht. Moga et al. verglichen die LSM von drei Untersuchern mit unterschiedlichen sonographischen und elastographischen Erfahrungsstufen und konnten eine exzellente Intra- und Interbeobachtervergleichbarkeit für das LOGIQ E9 nachweisen (Moga et al. 2018). Zudem verzichteten auch andere Arbeitsgruppen auf die Prüfung der Intra- bzw. Interbeobachtervariabilität (Goertz et al. 2012). In der vorliegenden Studie wurden alle Messungen von demselben Untersucher durchgeführt, was mögliche Fehlerquellen deutlich minimierte.

Eine weitere Limitation unserer Studie ist die begrenzte Anzahl an postprandialen Messungen, weswegen es uns nicht möglich ist, den Zeitraum zu bestimmen, an dem die LS wieder den Ausgangswert erreicht. Es wurde allerdings bereits gezeigt, dass der maximale Effekt der Nahrungsaufnahme auf die LS nach 30 bis 60 min eintritt (Goertz et al. 2012; Arena et al. 2013; Mederacke und Bahr 2014). Aufgrund dessen wurde in der vorliegenden Studie entschieden, eine einmalige Messung 30 bis 40 min nach Einnahme der Mahlzeit durchzuführen.

Außerdem ist zu erwähnen, dass die vorliegende Studie den Einfluss der Nahrungsaufnahme auf die LS ausschließlich an lebergesunden Probanden analysiert hat. Der Großteil des Studienkollektivs bestand aus sehr jungen und schlanken Probanden, die keinen bzw. nur geringen Alkoholkonsum angaben. Zudem litt kaum einer der Teilnehmer unter relevanten Vorerkrankungen. Dieses Profil entspricht nicht dem „klassischen“ internistischen und hepatologischen Patienten, bei dem eine Leberfibrose bzw. Zirrhose zu erwarten wäre. Möglicherweise sind unsere Ergebnisse somit nicht ohne weiteres auf leberkranke Patienten zu übertragen. Zudem geben unsere Daten Hinweise für geringere Auswirkungen der Einnahme einer Mahlzeit bei höheren LS-Ausgangswerten. Da bekannt ist, dass Patienten mit Leberfibrose höhere LS erreichen als gesunde Probanden, stellt sich somit die Frage, inwieweit die Nahrungsaufnahme hier einen signifikanten Einflussfaktor darstellt. Weitere Studien sind notwendig, um diesen Zusammenhang mittels 2D-SWE genauer zu untersuchen.

\subsection{Ausblick und Empfehlungen}

Die Normwerte für das Gerät LOGIQ E9 für lebergesunde Probanden lagen bei 4,80 $( \pm 0,94)$ kPa. Männer zeigten im Durchschnitt höhere LS als Frauen.

Der vermutete Einfluss der Nahrungsaufnahme auf die LS für die 2D-SWE konnte erstmals auch für das Gerät LOGIQ E9 bestätigt werden. Die LSM im Anschluss an die Einnahme einer Mahlzeit ergab einen durchschnittlichen Anstieg von 21,6\%.

Aufgrund der erhobenen Daten kann eine Fastenperiode von mindestens 3 h vor einer LSM empfohlen werden. Eine längere Fastenperiode scheint keinen signifikanten Einfluss mehr auf die LSM zu haben. Aufgrund der Nahrungsaufnahme wären 13 der gesunden Probanden 
mit einem Fibrosegrad $\mathrm{F} \geq 2$ fehlklassifiziert worden, wenn vorher kein Nüchternwert erhoben worden wäre. Somit wäre es bei diesen Probanden wahrscheinlich zu unnötigen und möglicherweise risikobehafteten weiteren diagnostischen Untersuchungen gekommen (z. B. Leberpunktion).

Trotzdem scheint es möglich zu sein, eine Zirrhose auszuschließen, wenn die gemessenen Werte - trotz stattgehabter Nahrungsaufnahme - im Normbereich liegen $(5,1( \pm 1,3) \mathrm{kPa}$; (Bende et al. 2017)). Falls dies nicht zutrifft, empfehlen wir die Messung nach einer Nüchternperiode von mindestens $3 \mathrm{~h}$ zu wiederholen.

Eine LS von über 7,5 kPa ist - bei nicht-nüchternen Patienten - nicht allein durch die Einnahme einer Mahlzeit zu begründen und sollte diagnostisch abgeklärt werden, da in diesen Fällen eine Leberfibrose vermutet werden muss.

Zudem wurde beobachtet, dass die Höhe des LS-Ausgangswerts den nahrungsbedingten LSA beeinflusst. Aufgrund dessen stellt sich die Frage, inwiefern die Nahrungsaufnahme bei Patienten mit hohen Ausgangswerten (wie zum Beispiel bei Patienten mit Leberzirrhose) zu berücksichtigen oder sogar zu vernachlässigen ist. Um dies zu untersuchen, werden nachfolgende Studien an Patienten mit chronischen Lebererkrankungen benötigt.

Da sich die vermutete Assoziation zwischen den Veränderungen der LS und des PBF statistisch nicht bestätigt hat, könnte die Untersuchung weiterer potenzieller Einflussfaktoren, wie des arteriell hepatischen Blutflusses und Veränderungen in der Zirkulation der Lebervenen, Gegenstand weiterführender Studien sein. 


\section{$5 \quad$ Zusammenfassung}

Chronische Lebererkrankungen führen zu einer Vermehrung der extrazellulären Matrix, aus der potenziell eine Fibrose und letztendlich eine Zirrhose hervorgehen kann. Aus einer progredienten Fibrosierung der Leber resultiert eine Änderung der Lebersteifigkeit, welche man mittels der nicht-invasiven Elastographie quantifizieren kann. Es existieren mehrere Elastographieverfahren (TE, p-SWE, 2D-SWE), zwischen denen ein direkter Vergleich nicht immer möglich ist, da unterschiedliche Techniken und Berechnungsmethoden verwendet werden. Alle Methoden basieren allerdings auf ähnlichen Verfahren, welche Scherwellen nutzen, um Veränderungen der Gewebssteifigkeit zu detektieren. Bei der Interpretation der Messwerte sind jedoch verschiedene Einflussfaktoren zu berücksichtigen. Einen dieser Einflussfaktoren stellt die Nahrungsaufnahme dar. Derzeit liegen keine publizierten Studien vor, die den Einfluss der Nahrungsaufnahme anhand der 2D-SWE (LOGIQ E9, GE) an gesunden Probanden untersuchen. Das Ziel der Studie ist es, den Einfluss der Nahrungsaufnahme auf die LS, gemessen mittels 2D-SWE (LOGIQ E9, GE), bei gesunden Probanden zu untersuchen und die erhobenen Messwerte mit dem PBF und anderen klinischen Parametern zu korrelieren.

Dafür wurde die LS und der PBF von 100 freiwilligen lebergesunden Probanden mit einer Fastenperiode von mindestens $3 \mathrm{~h}$ bis $>8 \mathrm{~h}$ vor und 30 bis 40 min nach Einnahme einer 800 kcal-Flüssigmahlzeit untersucht.

Die Nahrungsaufnahme führte postprandial zu signifikant höheren LS-Werten im Vergleich zu den präprandialen Messungen $(4,80( \pm 0,94) \mathrm{kPa}$ vs. 5,74 $( \pm 0,94) \mathrm{kPa})$. Dies entsprach einem Anstieg von 21,6\%.

Die Länge der Nahrungskarenz zeigte weder auf die präprandialen LS-Werte noch auf die nahrungsbedingten Veränderungen der LS einen signifikanten Effekt. Allerdings konnte ein Trend zu einem deutlicheren LSA bei Probanden mit einer Nüchternperiode von $>8 \mathrm{~h}$, im Vergleich zu 3 h bzw. 4 - 8 h, beobachtet werden.

Eine Abhängigkeit des nahrungsbedingten LSA vom Geschlecht oder dem Alter konnte nicht nachgewiesen werden. Auch die Einnahme von Medikamenten zeigte keinen Effekt, genauso wenig wie der durchschnittliche Tageskonsum von $<30 \mathrm{~g}$ Alkohol im Vergleich zu weniger bzw. gar keinem Alkoholkonsum. Studienteilnehmer mit einem höheren BMI erzielten im Durschnitt höhere LSA als Teilnehmer mit niedrigerem BMI. Des Weiteren zeigte die präprandiale LS eine negative Korrelation mit dem absoluten Anstieg der LS nach Nahrungsaufnahme. Eine eingeschränkte Validität der LSM nach Einnahme einer Mahlzeit wurde nicht nachgewiesen. Zudem zeigte sich ein signifikanter nahrungsbedingter Anstieg des PBF. Dieser korrelierte allerdings nicht mit dem beobachteten LSA. 
Die vorliegende Arbeit kommt somit zu dem Ergebnis, dass die Nahrungsaufnahme einen signifikanten Einflussfaktor der Elastographie darstellt. Aufgrund dessen empfehlen wir, eine Fastenperiode von mindestens 3 h vor Durchführung einer LSM einzuhalten.

Da die vermutete Assoziation zwischen den Veränderungen der LS und des PBF nicht belegt werden konnte, sollten weitere Studien durchgeführt werden, die mögliche ursächliche Faktoren wie die arterielle und venöse Blutzirkulation der Leber detaillierter untersuchen. 


\section{$6 \quad$ Anhang}

\section{UNIVERSITÄTSMEDIZIN $=\mathbf{U G}$
GÖTTINGEN $=\mathbf{V}$}

\section{Anamnesebogen für Probanden der klinischen Studie}

„Einfluss der Nahrungsaufnahme auf die Lebersteifigkeit, gemessen mit 2D-ScherwellenElastographie (Gerät: LOGIQ E9) bei freiwilligen Probanden“

\section{Durch den Probanden auszufüllen:}

\begin{tabular}{|c|c|c|c|c|c|c|c|}
\hline Geschlecht: & \multicolumn{3}{|c|}{$\square$ weiblich } & \multicolumn{4}{|c|}{$\square$ männlich } \\
\hline \multicolumn{8}{|l|}{ Geburtsdatum: } \\
\hline \multicolumn{8}{|l|}{ Größe [cm]: } \\
\hline \multicolumn{8}{|l|}{ Gewicht $[\mathrm{kg}]$ : } \\
\hline \multicolumn{2}{|c|}{ Lebererkrankungen? } & \multicolumn{2}{|c|}{$\square \mathrm{Ja}$} & \multicolumn{4}{|l|}{$\square$ Nein } \\
\hline \multicolumn{8}{|l|}{ Falls Ja, Welche?: } \\
\hline \multicolumn{4}{|c|}{ Medikamenteneinnahme?: $\quad \square \mathrm{Ja}$} & \multicolumn{4}{|c|}{$\square$ Nein } \\
\hline \multicolumn{8}{|l|}{ Falls Ja, Welche?: } \\
\hline Alkoholkonsum: & $\square 0 \mathrm{~g} / \mathrm{d}$ & & $-10 \mathrm{~g} / \mathrm{d}$ & $\square 10-$ & $0 \mathrm{~g} / \mathrm{d}$ & $\square 20-30 \mathrm{~g} / \mathrm{c}$ & $\square>30 \mathrm{~g} / \mathrm{d}$ \\
\hline \multicolumn{8}{|c|}{$\begin{array}{l}\text { Zur Orientierung: } \\
1 \text { Flasche Bier }(330 \mathrm{ml})=12,7 \mathrm{~g} \text { Alkohol } \\
1 \text { Glas Wein }(100 \mathrm{ml})=8,8 \mathrm{~g} \text { Alkohol } \\
1 \text { Glas Spirituose } 38 \%(20 \mathrm{ml})=6,1 \mathrm{~g} \text { Alkohol }\end{array}$} \\
\hline Nüchtern seit: & $\square 3 \mathrm{~h}$ & & $\square 4 \mathrm{~h}$ & & $\square 5 \mathrm{~h}$ & & $\Rightarrow 6 \mathrm{~h}$ \\
\hline$\square 7 \mathrm{~h}$ & $\square 8 \mathrm{~h}$ & & $\square 9 \mathrm{~h}$ & & $\square 10 \mathrm{r}$ & & $\Rightarrow 10 \mathrm{~h}$ \\
\hline
\end{tabular}

Hiermit bestätige ich die Richtigkeit und Vollständigkeit der gemachten Angaben: den, 


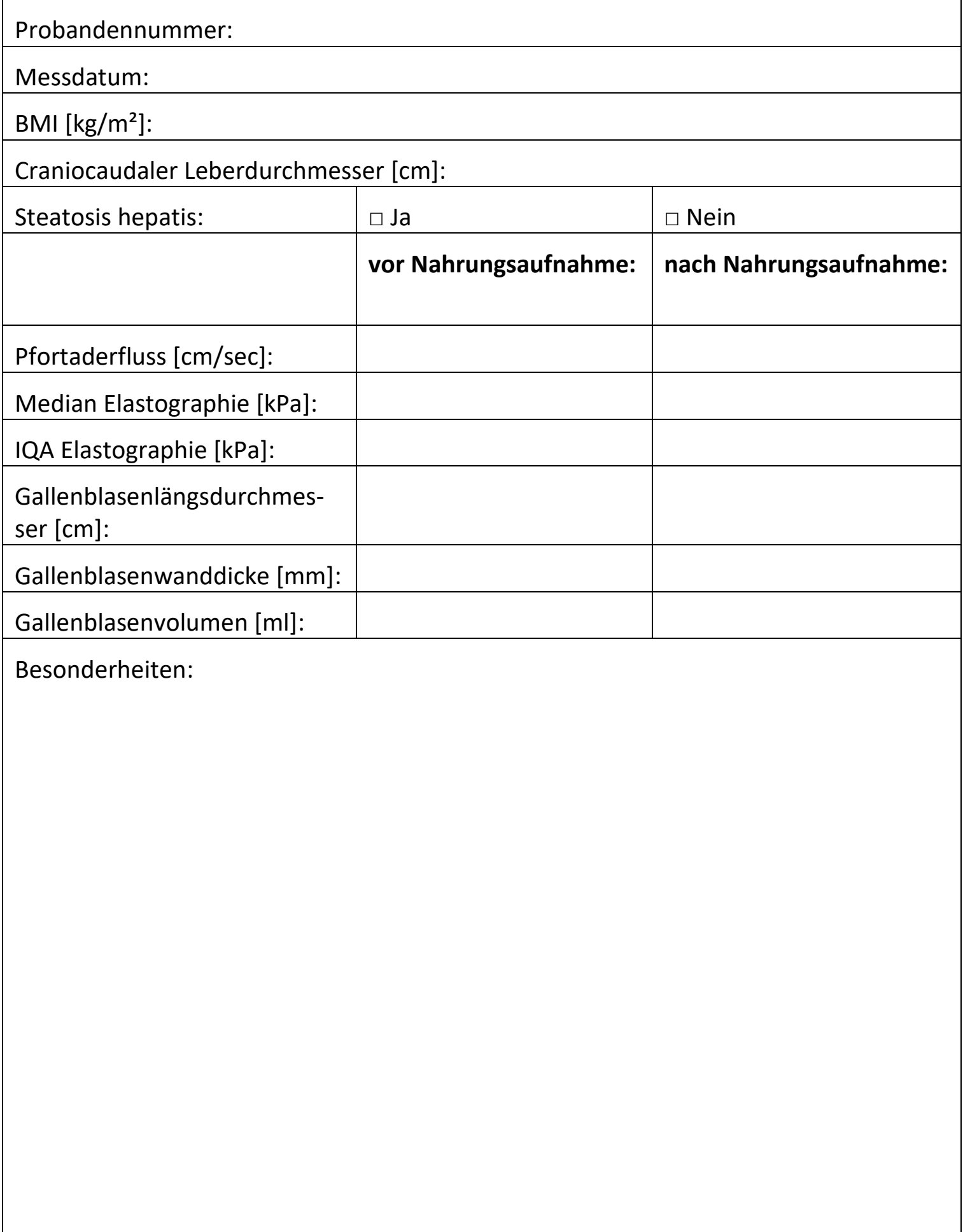

Abbildung 15: Probandenfragebogen. 


\section{UNIVERSITÄTSMEDIZIN $=\mathbf{U M G}$
GÖTTINGEN $=\mathbf{M G}$}

Universitätsmedizin Göttingen, 37099 Göttingen:
Klinik f. Gastroenterologie und gastrointestinale Onkologie, Robert-Koch-Straße 40, 37075 Göttingen

Direktor:

Briefpost: 37099 Göttingen

Adresse: Robert-Koch-Straße 40, 37075 Göttingen Telefon: 0551/39-66326

\section{Einwilligungserklärung}

Name

Geburtsdatum:

Das Original dieser Einwilligungserklärung verbleibt bei den Unterlagen. Eine Kopie der Einwilligungserklärung wird dem Probanden ausgehändigt.

Ich erkläre, dass ich die Probandeninformation zur wissenschaftlichen Untersuchung:

" Einfluss der Nahrungsaufnahme auf die Lebersteifigkeit "

und eine Kopie dieser Einwilligungserklärung erhalten habe.

Ich wurde für mich ausreichend mündlich und schriftlich über die wissenschaftliche Untersuchung informiert.

Ich weiß, dass ich jederzeit meine Einwilligung ohne Angaben von Gründen widerrufen kann, ohne dass dies für mich nachteilige Folgen hat.

$\square$ Ich bin damit einverstanden, dass die im Rahmen der wissenschaftlichen Untersuchung über mich erhobenen Daten sowie meine sonstigen mit dieser Untersuchung zusammenhängenden personenbezogenen Daten, insbesondere Gesundheitsdaten, aufgezeichnet werden. Es wird gewährleistet, dass meine personenbezogenen Daten nicht an Dritte weitergegeben werden. Bei der Veröffentlichung in einer wissenschaftlichen Zeitung wird aus den Daten nicht hervorgehen, wer an dieser Untersuchung

teilgenommen hat. Meine persönlichen Daten unterliegen dem Datenschutzgesetz.

Mit der vorstehend geschilderten Vorgehensweise bin ich einverstanden und bestätige dies mit meiner Unterschrift.

\begin{tabular}{llll}
\hline (Ort) & den, $\frac{\text { (Proband) }}{}$ \\
& den, $\frac{}{\text { (Datum) }}$ (Prüfarzt)
\end{tabular}

Abbildung 16: Einwilligungserklärung der Studienteilnehmer. 


\section{$7 \quad$ Literaturverzeichnis}

Alvarez D, Orozco F, Mella JM, Anders M, Antinucci F, Mastai R (2015): Meal ingestion markedly increases liver stiffness suggesting the need for liver stiffness determination in fasting conditions. Gastroenterol Hepatol $\underline{38}$, 431-435

Arena U, Vizzutti F, Corti G, Ambu S, Stasi C, Bresci S, Moscarella S, Boddi V, Petrarca A, Laffi G, et al. (2008): Acute viral hepatitis increases liver stiffness values measured by transient elastography. Hepatol 47, 380-384

Arena U, Lupsor Platon M, Stasi C, Moscarella S, Assarat A, Bedogni G, Piazzolla V, Badea R, Laffi G, Marra F, et al. (2013): Liver stiffness is influenced by a standardized meal in patients with chronic hepatitis $\mathrm{C}$ virus at different stages of fibrotic evolution. Hepatol $\underline{58}$, $65-72$

Aubé C, Oberti F, Korali N, Namour MA, Loisel D, Tanguy JY, Valsesia E, Pilette C, Rousselet MC, Bedossa P, et al. (1999): Ultrasonographic diagnosis of hepatic fibrosis or cirrhosis. J Hepatol $\underline{30}, 472-478$

Bamber J, Cosgrove D, Dietrich CF, Fromageau J, Bojunga J, Calliada F, Cantisani V, Correas J-M, D’Onofrio M, Drakonaki EE, et al. (2013): EFSUMB guidelines and recommendations on the clinical use of ultrasound elastography. Part 1: Basic principles and technology. Ultraschall Med $\underline{34}, 169-184$

Bardou-Jacquet E, Legros L, Soro D, Latournerie M, Guillygomarc'h A, Le Lan C, Brissot P, Guyader D, Moirand R (2013): Effect of alcohol consumption on liver stiffness measured by transient elastography. World J Gastroenterol $\underline{19}, 516-522$

Barone M, Iannone A, Brunetti ND, Sebastiani F, Cecere O, Berardi E, Antonica G, Di Leo A (2015): Liver stiffness and portal blood flow modifications induced by a liquid meal consumption: pathogenetic mechanisms and clinical relevance. Scand J Gastroenterol $\underline{50}, 560$ 566

Barr RG (2018): Shear wave liver elastography. Abdom Radiol (NY) $\underline{43}$, 800-807

Baunsgaard P, Sanchez GC, Lundborg CJ (1979): The variation of pathological changes in the liver evaluated by double biopsies. Acta Pathol Microbiol Scand A. $\underline{87}, 51-57$

Bedossa P, Dargère D, Paradis V (2003): Sampling variability of liver fibrosis in chronic hepatitis C. Hepatol $\underline{38}, 1449-1457$

Bende F, Sporea I, Sirli R, Popescu A, Mare R, Miutescu B, Lupusoru R, Moga T, Pienar C (2017): Performance of 2D-SWE.GE for predicting different stages of liver fibrosis, using Transient Elastography as the reference method. Med Ultrason $\underline{19}, 143-149$

Bende F, Mulabecirovic A, Sporea I, Popescu A, Sirli R, Gilja OH, Vesterhus M, Havre RF (2018): Assessing Liver Stiffness by 2-D Shear Wave Elastography in a Healthy Cohort. 
Ultrasound Med Biol 44, 332-341

Berzigotti A, De Gottardi A, Vukotic R, Siramolpiwat S, Abraldes JG, García-Pagan JC, Bosch J (2013): Effect of meal ingestion on liver stiffness in patients with cirrhosis and portal hypertension. PloS One $\underline{8}$, e58742

Boursier J, Konaté A, Gorea G, Reaud S, Quemener E, Oberti F, Hubert-Fouchard I, Dib N, Calès P (2008): Reproducibility of liver stiffness measurement by ultrasonographic elastometry. Clin Gastroenterol Hepatol $\underline{6}, 1263-1269$

Castéra L, Vergniol J, Foucher J, Le Bail B, Chanteloup E, Haaser M, Darriet M, Couzigou P, De Lédinghen V (2005): Prospective comparison of transient elastography, Fibrotest, APRI, and liver biopsy for the assessment of fibrosis in chronic hepatitis C. Gastroenterology $\underline{128}, 343-350$

Castera L, Forns X, Alberti A (2008): Non-invasive evaluation of liver fibrosis using transient elastography. J Hepatol $\underline{48}, 835-847$

Coco B, Oliveri F, Maina AM, Ciccorossi P, Sacco R, Colombatto P, Bonino F, Brunetto MR (2007): Transient elastography: a new surrogate marker of liver fibrosis influenced by major changes of transaminases. J Viral Hepat 14, 360-369

Colak Y, Bozbey G, Erim T, Caklili OT, Ulasoglu C, Senates E, Mutlu HH, Mesci B, Doğan MS, Tasan G, et al. (2016): Impaired Gallbladder Motility and Increased Gallbladder Wall Thickness in Patients with Nonalcoholic Fatty Liver Disease. J Neurogastroenterol Motil $\underline{22}$, $470-476$

Colli A, Fraquelli M, Andreoletti M, Marino B, Zuccoli E, Conte D (2003): Severe liver fibrosis or cirrhosis: accuracy of US for detection--analysis of 300 cases. Radiology 227, 89-94

Colli A, Pozzoni P, Berzuini A, Gerosa A, Canovi C, Molteni EE, Barbarini M, Bonino F, Prati D (2010): Decompensated chronic heart failure: increased liver stiffness measured by means of transient elastography. Radiology 257, 872-878

Colombo S, Belloli L, Zaccanelli M, Badia E, Jamoletti C, Buonocore M, Del Poggio P (2011): Normal liver stiffness and its determinants in healthy blood donors. Dig Liver Dis $\underline{43}, 231-236$

Cosgrove D, Piscaglia F, Bamber J, Bojunga J, Correas J-M, Gilja OH, Klauser AS, Sporea I, Calliada F, Cantisani V, et al. (2013): EFSUMB guidelines and recommendations on the clinical use of ultrasound elastography. Part 2: Clinical applications. Ultraschall Med $\underline{34}$, $238-253$

Crownover BK, Covey CJ (2013): Hereditary hemochromatosis. Am Fam Physician $\underline{87}$, 183-190

Dauzat M, Lafortune M, Patriquin H, Pomier-Layrargues G (1994): Meal induced changes 
in hepatic and splanchnic circulation: a noninvasive Doppler study in normal humans. Eur J Appl Physiol Occup Physiol 68, 373-380

Dietrich CF, Bamber J, Berzigotti A, Bota S, Cantisani V, Castera L, Cosgrove D, Ferraioli G, Friedrich-Rust M, Gilja OH, et al. (2017): EFSUMB Guidelines and Recommendations on the Clinical Use of Liver Ultrasound Elastography, Update 2017 (Long Version). Ultraschall Med $\underline{38}$, e16-e47

Elpek GÖ (2014): Cellular and molecular mechanisms in the pathogenesis of liver fibrosis: An update. World J Gastroenterol 20, 7260-7276

Fang C, Konstantatou E, Romanos O, Yusuf GT, Quinlan DJ, Sidhu PS (2017): Reproducibility of 2-Dimensional Shear Wave Elastography Assessment of the Liver: A Direct Comparison With Point Shear Wave Elastography in Healthy Volunteers. J Ultrasound Med $\underline{36}$, 1563-1569

Ferraioli G, Tinelli C, Dal Bello B, Zicchetti M, Filice G, Filice C, Liver Fibrosis Study Group (2012): Accuracy of real-time shear wave elastography for assessing liver fibrosis in chronic hepatitis C: a pilot study. Hepatol $\underline{56}$, 2125-2133

Ferraioli G, Tinelli C, Lissandrin R, Zicchetti M, Dal Bello B, Filice G, Filice C (2014): Point shear wave elastography method for assessing liver stiffness. World J Gastroenterol $\underline{20}, 4787-4796$

Ferraioli G, Filice C, Castera L, Choi BI, Sporea I, Wilson SR, Cosgrove D, Dietrich CF, Amy D, Bamber JC, et al. (2015): WFUMB guidelines and recommendations for clinical use of ultrasound elastography: Part 3: liver. Ultrasound Med Biol 41, 1161-1179

Franckenberg S, Gubler C, Frauenfelder T, Rominger M (2016): [Radiology Update Ultrasound Elastography - Quintessence for the Primary Care Physician]. Praxis (Bern 1994) $\underline{105}, 139-145$

Fraquelli M, Rigamonti C, Casazza G, Conte D, Donato MF, Ronchi G, Colombo M (2007): Reproducibility of transient elastography in the evaluation of liver fibrosis in patients with chronic liver disease. Gut $\underline{56}$, 968-973

Friedrich-Rust M, Vermehren J (2013): [Nicht-invasive Verfahren zur Bestimmung der Leberfibrose in der klinischen Praxis]. Z Gastroenterol 51, 43-54

Friedrich-Rust M, Ong M-F, Martens S, Sarrazin C, Bojunga J, Zeuzem S, Herrmann E (2008): Performance of transient elastography for the staging of liver fibrosis: a meta-analysis. Gastroenterology 134, 960-974

Friedrich-Rust M, Wunder K, Kriener S, Sotoudeh F, Richter S, Bojunga J, Herrmann E, Poynard T, Dietrich CF, Vermehren J, et al. (2009): Liver fibrosis in viral hepatitis: noninvasive assessment with acoustic radiation force impulse imaging versus transient elastography. Radiology 252, 595-604 
Frulio N, Trillaud H (2013): Ultrasound elastography in liver. Diagn Interv Imaging 94, 515534

Gaiani S, Bolondi L, Li Bassi S, Santi V, Zironi G, Barbara L (1989): Effect of meal on portal hemodynamics in healthy humans and in patients with chronic liver disease. Hepatol $\underline{9}, 815-819$

Garra BS (2015): Elastography: history, principles, and technique comparison. Abdom Imaging $\underline{40}, 680-697$

GE Healthcare (2014): https://www3.gehealthcare.com/ /media/rsna-2016-press-kit-assets/press\%20releases/ultrasound/global\%20shear\%20wave\%20whitepaper_oc-

tober\%202014.pdf?Parent=\%7B1FFF8A39-2AC6-4EA6-9119-7458819CB4F7\%7D; Zugriff am 07.02.2018

Gekle M: Funktion des Magen-Darm-Trakts, Energiehaushalt und Ernährung. In: Klinke R, Pape H, Kurtz A, Silbernagl S (Hrsg.): Physiologie. 6. Auflage; Georg Thieme Verlag, Stuttgart 2009, 416-499

Gersak MM, Badea R, Lenghel LM, Vasilescu D, Botar-Jid C, Dudea SM (2016a): Influence of Food Intake on 2-D Shear Wave Elastography Assessment of Liver Stiffness in Healthy Subjects. Ultrasound Med Biol $\underline{42}$, 1295-1302

Gersak MM, Sorantin E, Windhaber J, Dudea SM, Riccabona M (2016b): The influence of acute physical effort on liver stiffness estimation using Virtual Touch Quantification (VTQ). Preliminary results. Med Ultrason $\underline{18}, 151-156$

Goertz RS (2015): [Ultraschallelastographie]. Radiol 55, 949-955

Goertz RS, Egger C, Neurath MF, Strobel D (2012): Impact of food intake, ultrasound transducer, breathing maneuvers and body position on acoustic radiation force impulse (ARFI) elastometry of the liver. Ultraschall Med $\underline{33}, 380-385$

Grgurević I, Bokun T, Mustapić S, Trkulja V, Heinzl R, Banić M, Puljiz Ž, Lukšić B, Kujundžić M (2015): Real-time two-dimensional shear wave ultrasound elastography of the liver is a reliable predictor of clinical outcomes and the presence of esophageal varices in patients with compensated liver cirrhosis. Croat Med J $\underline{56}, 470-481$

Guyot C, Lepreux S, Combe C, Doudnikoff E, Bioulac-Sage P, Balabaud C, Desmoulière A (2006): Hepatic fibrosis and cirrhosis: the (myo)fibroblastic cell subpopulations involved. Int J Biochem Cell Biol $\underline{38}, 135-151$

Guzmán-Aroca F, Reus M, Berná-Serna JD, Serrano L, Serrano C, Gilabert A, Cepero A (2011): Reproducibility of shear wave velocity measurements by acoustic radiation force impulse imaging of the liver: a study in healthy volunteers. J Ultrasound Med $\underline{30}$, 975-979

Horster S, Mandel P, Zachoval R, Clevert DA (2010): Comparing acoustic radiation force impulse imaging to transient elastography to assess liver stiffness in healthy volunteers with 
and without valsalva manoeuvre. Clin Hemorheol Microcirc $\underline{46}$, 159-168

Huang Z, Zheng J, Zeng J, Wang X, Wu T, Zheng R (2014): Normal liver stiffness in healthy adults assessed by real-time shear wave elastography and factors that influence this method. Ultrasound Med Biol 40, 2549-2555

Jiang J, Hall TJ (2015): A coupled subsample displacement estimation method for ultrasound-based strain elastography. Phys Med Biol $\underline{60}$, 8347-8364

Jiang T, Tian G, Zhao Q, Kong D, Cheng C, Zhong L, Li L (2016): Diagnostic Accuracy of 2D-Shear Wave Elastography for Liver Fibrosis Severity: A Meta-Analysis. PloS One 11, e0157219

Kendall TJ, Hennedige S, Aucott RL, Hartland SN, Vernon MA, Benyon RC, Iredale JP (2009): p75 Neurotrophin receptor signaling regulates hepatic myofibroblast proliferation and apoptosis in recovery from rodent liver fibrosis. Hepatol $\underline{49}, 901-910$

Kim SU, Choi GH, Han WK, Kim BK, Park JY, Kim DY, Choi JS, Yang SC, Choi EH, Ahn SH, et al. (2010): What are „true normal“ liver stiffness values using FibroScan?: a prospective study in healthy living liver and kidney donors in South Korea. Liver Int 30, 268-274

Kjærgaard M, Thiele M, Jansen C, Stæhr Madsen B, Görtzen J, Strassburg C, Trebicka J, Krag A (2017): High risk of misinterpreting liver and spleen stiffness using 2D shear-wave and transient elastography after a moderate or high calorie meal. PloS One $\underline{12}$, e0173992

Lemoine M, Shimakawa Y, Njie R, Njai HF, Nayagam S, Khalil M, Goldin R, Ingiliz P, Taal M, Nyan O, et al. (2014): Food intake increases liver stiffness measurements and hampers reliable values in patients with chronic hepatitis B and healthy controls: the PROLIFICA experience in The Gambia. Aliment Pharmacol Ther 39, 188-196

Leung VY, Shen J, Wong VW, Abrigo J, Wong GL, Chim AM, Chu SH, Chan AW, Choi PC, Ahuja AT, et al. (2013): Quantitative elastography of liver fibrosis and spleen stiffness in chronic hepatitis B carriers: comparison of shear-wave elastography and transient elastography with liver biopsy correlation. Radiology $\underline{269}, 910-918$

Liao L-Y, Kuo K-L, Chiang H-S, Lin C-Z, Lin Y-P, Lin C-L (2015): Acoustic radiation force impulse elastography of the liver in healthy patients: test location, reference range and influence of gender and body mass index. Ultrasound Med Biol 411, 698-704

Ling W, Lu Q, Quan J, Ma L, Luo Y (2013): Assessment of impact factors on shear wave

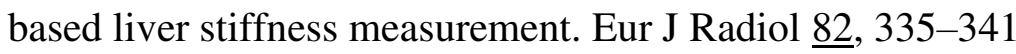

Ludwig D, Schwarting K, Korbel CM, Brüning A, Schiefer B, Stange EF (1998): The postprandial portal flow is related to the severity of portal hypertension and liver cirrhosis. $\mathbf{J}$ Hepatol 28, 631-638

Macaluso FS, Maida M, Cammà C, Cabibbo G, Cabibi D, Alduino R, Di Marco V, Craxì A, Petta S (2014): Steatosis affects the performance of liver stiffness measurement for fibrosis 
assessment in patients with genotype 1 chronic hepatitis C. J Hepatol $\underline{61}$, 523-529

Madhok R, Tapasvi C, Prasad U, Gupta AK, Aggarwal A (2013): Acoustic radiation force impulse imaging of the liver: measurement of the normal mean values of the shearing wave velocity in a healthy liver. J Clin Diagn Res $\underline{7}, 39-42$

Mathurin P, Hadengue A, Bataller R, Addolorato G, Burra P, Burt A, Caballeria J, CortezPinto H, Day CP, Forrest EH, et al. (2012): EASL clinical practical guidelines: management of alcoholic liver disease. J Hepatol $\underline{57}$, 399-420

Mederacke I, Bahr MJ (2014): Liver stiffness after meal intake at different stages of fibrotic evolution. Hepatol 59, 734-735

Mederacke I, Wursthorn K, Kirschner J, Rifai K, Manns MP, Wedemeyer H, Bahr MJ (2009): Food intake increases liver stiffness in patients with chronic or resolved hepatitis $\mathrm{C}$ virus infection. Liver Int 29, 1500-1506

Millonig G, Reimann FM, Friedrich S, Fonouni H, Mehrabi A, Büchler MW, Seitz HK, Mueller S (2008): Extrahepatic cholestasis increases liver stiffness (FibroScan) irrespective of fibrosis. Hepatol $\underline{48}, 1718-1723$

Millonig G, Friedrich S, Adolf S, Fonouni H, Golriz M, Mehrabi A, Stiefel P, Pöschl G, Büchler MW, Seitz HK, Mueller S (2010): Liver stiffness is directly influenced by central venous pressure. J Hepatol $\underline{52}$, 206-210

Moga T-V, Stepan AM, Pienar C, Bende F, Popescu A, Șirli R, Dănilă M, Sporea I (2018): Intra- and Inter-Observer Reproducibility of a 2-D Shear Wave Elastography Technique and the Impact of Ultrasound Experience in Achieving Reliable Data. Ultrasound Med Biol $\underline{44}$, $1627-1637$

Moneta GL, Taylor DC, Helton WS, Mulholland MW, Strandness DE (1988): Duplex ultrasound measurement of postprandial intestinal blood flow: effect of meal composition. Gastroenterology $\underline{95}, 1294-1301$

Mueller S (2016): Does pressure cause liver cirrhosis? The sinusoidal pressure hypothesis. World J Gastroenterol 22, 10482-10501

Mueller S, Millonig G, Sarovska L, Friedrich S, Reimann FM, Pritsch M, Eisele S, Stickel F, Longerich T, Schirmacher P, Seitz HK (2010): Increased liver stiffness in alcoholic liver disease: differentiating fibrosis from steatohepatitis. World J Gastroenterol 16, 966-972

Mulabecirovic A, Mjelle AB, Gilja OH, Vesterhus M, Havre RF (2018): Repeatability of shear wave elastography in liver fibrosis phantoms-Evaluation of five different systems. PloS One $\underline{13}$, e0189671

Mutimer D, Aghemo A, Diepolder H, Negro F, Robaeys G, Ryder S, Zoulim F (2014): EASL Clinical Practice Guidelines: management of hepatitis C virus infection. J Hepatol $\underline{60}$, 392420 
Nightingale K, McAleavey S, Trahey G (2003): Shear-wave generation using acoustic radiation force: in vivo and ex vivo results. Ultrasound Med Biol 29, 1715-1723

Numata K, Tanaka K, Kiba T, Morita K, Saito S, Fujii T, Sekihara H (1999): Hepatic arterial resistance after mixed-meal ingestion in healthy subjects and patients with chronic liver disease. J Clin Ultrasound 27, 239-248

Petta S, Maida M, Macaluso FS, Di Marco V, Cammà C, Cabibi D, Craxì A (2015): The severity of steatosis influences liver stiffness measurement in patients with nonalcoholic fatty liver disease. Hepatol $\underline{62}, 1101-1110$

Pinzani M, Macias-Barragan J (2010): Update on the pathophysiology of liver fibrosis. Expert Rev Gastroenterol Hepatol 4, 459-472

Piscaglia F, Salvatore V, Mulazzani L, Cantisani V, Schiavone C (2016): Ultrasound Shear Wave Elastography for Liver Disease. A Critical Appraisal of the Many Actors on the Stage. Ultraschall Med $\underline{37}, 1-5$

Popescu A, Sporea I, Sirli R, Bota S, Focşa M, Dănilă M, Nicoliţă D, Martie A, Sendroiu M, Juchiş A (2011): The mean values of liver stiffness assessed by Acoustic Radiation Force Impulse elastography in normal subjects. Med Ultrason $\underline{13}, 33-37$

Popescu A, Bota S, Sporea I, Sirli R, Danila M, Racean S, Suseanu D, Gradinaru O, Ivascu Siegfried C (2013): The influence of food intake on liver stiffness values assessed by acoustic radiation force impulse elastography-preliminary results. Ultrasound Med Biol 39, 579 584

Popescu A, Lupusoru R, Bende F, Mare R, Sirli R, Danila M, Moga T, Pienar C, Stepan A, Sporea I (2016): The influence of food intake on liver stiffness measurements obtained by two 2D-SWE methods. Ultraschall Med $\underline{37}$

Poujois A, Woimant F (2018): Wilson's disease: A 2017 update. Clin Res Hepatol Gastroenterol $\underline{42}, 512-520$

Poupon R (2015): Non-Invasive Assessment of Liver Fibrosis Progression and Prognosis in Primary Biliary Cholangitis. Dig Dis Suppl 2, 115-117

Ratchatasettakul K, Rattanasiri S, Promson K, Sringam P, Sobhonslidsuk A (2017): The inverse effect of meal intake on controlled attenuation parameter and liver stiffness as assessed by transient elastography. BMC Gastroenterol $\underline{17}, 50$

Regev A, Berho M, Jeffers LJ, Milikowski C, Molina EG, Pyrsopoulos NT, Feng Z-Z, Reddy KR, Schiff ER (2002): Sampling error and intraobserver variation in liver biopsy in patients with chronic HCV infection. Am J Gastroenterol 97, 2614-2618

Reiberger T, Ferlitsch A, Payer BA, Pinter M, Homoncik M, Peck-Radosavljevic M, Vienna Hepatic Hemodynamic Lab (2012): Non-selective $\beta$-blockers improve the correlation of liver stiffness and portal pressure in advanced cirrhosis. J Gastroenterol $\underline{47}, 561-568$ 
Rizzo L, Calvaruso V, Cacopardo B, Alessi N, Attanasio M, Petta S, Fatuzzo F, Montineri A, Mazzola A, L'abbate L, et al. (2011): Comparison of transient elastography and acoustic radiation force impulse for non-invasive staging of liver fibrosis in patients with chronic hepatitis C. Am J Gastroenterol 106, 2112-2120

Rockey DC, Caldwell SH, Goodman ZD, Nelson RC, Smith AD, American Association for the Study of Liver Diseases (2009): Liver biopsy. Hepatol 499, 1017-1044

Roeb E, Steffen HM, Bantel H, Baumann U, Canbay A, Demir M, Drebber U, Geier A, Hampe J, Hellerbrand C, et al. (2015): [S2k Leitlinie für die nicht-alkoholische Fettlebererkrankung]. Z Gastroenterol 53, 668-723

Sabbá C, Ferraioli G, Genecin P, Colombato L, Buonamico P, Lerner E, Taylor KJ, Groszmann RJ (1991): Evaluation of postprandial hyperemia in superior mesenteric artery and portal vein in healthy and cirrhotic humans: an operator-blind echo-Doppler study. Hepatol $\underline{13}, 714-718$

Sagir A, Erhardt A, Schmitt M, Häussinger D (2008): Transient elastography is unreliable for detection of cirrhosis in patients with acute liver damage. Hepatol 47, 592-595

Sandrin L, Fourquet B, Hasquenoph J-M, Yon S, Fournier C, Mal F, Christidis C, Ziol M, Poulet B, Kazemi F, et al. (2003): Transient elastography: a new noninvasive method for assessment of hepatic fibrosis. Ultrasound Med Biol 29, 1705-1713

Schiedermaier P, Koch L, Mojón A, Hermida R, Layer G, Sauerbruch T (2006): Circadian rhythm of fasting and postprandial portal blood flow in cirrhosis. Scand J Gastroenterol $\underline{41}$, 826-832

Sebastiani G, Gkouvatsos K, Pantopoulos K (2014): Chronic hepatitis C and liver fibrosis. World J Gastroenterol 20, 11033-11053

Serra C, Grasso V, Conti F, Felicani C, Mazzotta E, Lenzi M, Verucchi G, D'errico A, Andreone P (2018): A New Two-Dimensional Shear Wave Elastography for Noninvasive Assessment of Liver Fibrosis in Healthy Subjects and in Patients with Chronic Liver Disease. Ultraschall Med $\underline{39}$, 432-439

Sirli R, Bota S, Sporea I, Jurchis A, Popescu A, Gradinaru-Tascău O, Szilaski M (2013): Liver stiffness measurements by means of supersonic shear imaging in patients without known liver pathology. Ultrasound Med Biol 39, 1362-1367

Son CY, Kim SU, Han WK, Choi GH, Park H, Yang SC, Choi JS, Park JY, Kim DY, Ahn $\mathrm{SH}$, et al. (2012): Normal liver elasticity values using acoustic radiation force impulse imaging: a prospective study in healthy living liver and kidney donors. J Gastroenterol Hepatol $\underline{27}, 130-136$

Sporea I, Bota S, Peck-Radosavljevic M, Sirli R, Tanaka H, Iijima H, Badea R, Lupsor M, 
Fierbinteanu-Braticevici C, Petrisor A, et al. (2012): Acoustic Radiation Force Impulse elastography for fibrosis evaluation in patients with chronic hepatitis $\mathrm{C}$ : an international multicenter study. Eur J Radiol 81, 4112-4118

Strobel D, Bernatik T, Blank W, Will U, Reichel A, Wüstner M, Keim V, Schacherer D, Barreiros AP, Kunze G, et al. (2015): Incidence of bleeding in 8172 percutaneous ultrasound-guided intraabdominal diagnostic and therapeutic interventions - results of the prospective multicenter DEGUM interventional ultrasound study (PIUS study). Ultraschall Med $\underline{36}, 122-131$

Suh CH, Kim SY, Kim KW, Lim Y-S, Lee SJ, Lee M-G, Lee J, Lee S-G, Yu E (2014): Determination of normal hepatic elasticity by using real-time shear-wave elastography. Radiology $\underline{271}, 895-900$

Sun J, Cai J, Wang X (2014): Real-time ultrasound elastography for differentiation of benign and malignant thyroid nodules: a meta-analysis. J Ultrasound Med 33, 495-502

Takahashi H, Ono N, Eguchi Y, Eguchi T, Kitajima Y, Kawaguchi Y, Nakashita S, Ozaki I, Mizuta T, Toda S, et al. (2010): Evaluation of acoustic radiation force impulse elastography for fibrosis staging of chronic liver disease: a pilot study. Liver Int $\underline{30}, 538-545$

Talwalkar JA, Kurtz DM, Schoenleber SJ, West CP, Montori VM (2007): Ultrasound-based transient elastography for the detection of hepatic fibrosis: systematic review and meta-analysis. Clin Gastroenterol Hepatol $\underline{5}, 1214-1220$

Treece G, Lindop J, Chen L, Housden J, Prager R, Gee A (2011): Real-time quasi-static ultrasound elastography. Interface Focus $\underline{1}, 540-552$

Tsochatzis EA, Gurusamy KS, Ntaoula S, Cholongitas E, Davidson BR, Burroughs AK (2011): Elastography for the diagnosis of severity of fibrosis in chronic liver disease: a metaanalysis of diagnostic accuracy. J Hepatol $\underline{54}, 650-659$

Wang H, Naghavi M, Allen C, Barber RM, Bhutta ZA, Carter A, Casey DC, Charlson FJ, Chen AZ, Coates MM, et al. (2016): Global, regional, and national life expectancy, all-cause mortality, and cause-specific mortality for 249 causes of death, 1980-2015: a systematic analysis for the Global Burden of Disease Study 2015. Lancet $\underline{388}$, 1459-1544

Wilkins RH (1964): neurosurgical classic. XVII. J Neurosurg 21, 1108-1114

Wong VW-S, Vergniol J, Wong GL-H, Foucher J, Chan HL-Y, Le Bail B, Choi PC-L, Kowo M, Chan AW-H, Merrouche W, et al. (2010): Diagnosis of fibrosis and cirrhosis using liver stiffness measurement in nonalcoholic fatty liver disease. Hepatol $\underline{51}, 454-462$

Wood AM, Kaptoge S, Butterworth AS, Willeit P, Warnakula S, Bolton T, Paige E, Paul DS, Sweeting M, Burgess S, et al. (2018): Risk thresholds for alcohol consumption: combined analysis of individual-participant data for 599912 current drinkers in 83 prospective studies. Lancet $\underline{391}, 1513-1523$ 
Yoneda M, Yoneda M, Mawatari H, Fujita K, Endo H, lida H, Nozaki Y, Yonemitsu K, Higurashi T, Takahashi H, et al. (2008): Noninvasive assessment of liver fibrosis by measurement of stiffness in patients with nonalcoholic fatty liver disease (NAFLD). Dig Liver Dis $\underline{40}, 371-378$

Zardi EM, Dobrina A, Uwechie V, Cacciapaglia F, Rollo M, Laghi V, Ambrosino G, Lumachi F (2008): Postmeal portal flow variations in HCV-related chronic hepatitis and liver cirrhosis with and without hyperdynamic syndrome. In Vivo 22, 509-512 


\section{Danksagung}

Ich bedanke mich ganz herzlich bei Herrn PD Dr. Dr. Albrecht Neeße für die Überlassung des spannenden Dissertationsthemas, für die engagierte und kompetente Betreuung und vor allem für die ständige Erreichbarkeit bei Fragen oder Problemen.

Mein besonderer Dank gilt Herrn Dr. Golo Petzold, der mich von der Durchführung der Studie bis zur Verfassung der vorliegenden Arbeit stets intensiv unterstützt hat. Außerdem danke ich ihnen für die kompetente Einarbeitung, die Hilfestellung bei der Verfassung der Dissertation und ihr immer offenes Ohr, ohne die meine Arbeit in einem solchen Rahmen nicht möglich gewesen wäre.

Den Mitarbeitern des Instituts für Medizinische Statistik der Universitätsmedizin Göttingen danke ich für die Unterstützung bei der statistischen Auswertung der Daten.

Zudem möchte ich mich bei allen 100 Probanden für die Teilnahme und für die Beantwortung der Fragen bedanken. 


\section{Lebenslauf}

Am 20.09.1993 wurde ich als erstes Kind des Technikers Adrian Przygoda-Porsche und der Erzieherin Dagmar Porsche in Bochum geboren. Ich wuchs zusammen mit meinem jüngeren Bruder Mauriz Porsche (geboren 1998) in Bochum auf und lebte dort bis zum Ende meiner Schulzeit. Ich besuchte die Hans-Christian-Anderson-Grundschule von 2000 bis 2004 und das Heinrich-von-Kleist-Gymnasium von 2004 bis zum Erhalt der allgemeinen Hochschulreife im Jahre 2013. Im November 2013 begann ich mein Medizinstudium an der Georg-August-Universität in Göttingen. Den Ersten Abschnitt der Ärztlichen Prüfung absolvierte ich im September 2015 mit der Gesamtnote gut $(2,0)$. In dem darauffolgenden klinischen Studienabschnitt begann ich im Jahre 2016 meine Tätigkeit als studentische Hilfskraft im ,studentischen Trainingszentrum Ärztlicher Praxis und Simulation“ (STÄPS) der Georg-August-Universität unter der Leitung von Frau Prof. Dr. Anne Simmenroth. Ich engagierte mich dort besonders in den Bereichen Gynäkologie, Hals-Nasen-Ohren-Heilkunde, Sonographie und der Lehre chirurgischer Fertigkeiten. Zudem leistete ich Nachtdienste im Evangelischen Krankenhaus Göttingen-Weende und beteiligte mich als studentische Hilfskraft an dem „Göttinger Repetitorium Vorklinik“ (GRV).

Im Sommer 2017 fanden die Vorbereitungen zu der hier vorliegenden Arbeit statt, bis ich Ende August 2017 mit der praktischen Durchführung der Studie in der Klinik für Gastroenterologie und gastrointestinale Onkologie unter der Leitung von Herrn PD Dr. Dr. Albrecht Neeße und der Betreuung durch Herrn Dr. Golo Petzold begann. Im Zeitraum von August bis Dezember 2017 fand die Datenerhebung statt, woraufhin die Verschriftlichung der vorliegenden Arbeit folgte.

Im Oktober 2018 legte ich die Zweite Ärztliche Prüfung mit der Gesamtnote gut ab und befinde mich nun seit November 2018 im Praktischen Jahr. 